\title{
Pre-randomization in study designs acceptability and applicability
}

Citation for published version (APA):

Schellings, A. G. L. (2005). Pre-randomization in study designs acceptability and applicability. [Doctoral Thesis, Maastricht University]. https://doi.org/10.26481/dis.20051007as

Document status and date:

Published: 01/01/2005

DOI:

10.26481/dis.20051007as

Document Version:

Publisher's PDF, also known as Version of record

\section{Please check the document version of this publication:}

- A submitted manuscript is the version of the article upon submission and before peer-review. There can be important differences between the submitted version and the official published version of record.

People interested in the research are advised to contact the author for the final version of the publication, or visit the DOI to the publisher's website.

- The final author version and the galley proof are versions of the publication after peer review.

- The final published version features the final layout of the paper including the volume, issue and page numbers.

Link to publication

\footnotetext{
General rights rights.

- You may freely distribute the URL identifying the publication in the public portal. please follow below link for the End User Agreement:

www.umlib.nl/taverne-license

Take down policy

If you believe that this document breaches copyright please contact us at:

repository@maastrichtuniversity.nl

providing details and we will investigate your claim.
}

Copyright and moral rights for the publications made accessible in the public portal are retained by the authors and/or other copyright owners and it is a condition of accessing publications that users recognise and abide by the legal requirements associated with these

- Users may download and print one copy of any publication from the public portal for the purpose of private study or research.

- You may not further distribute the material or use it for any profit-making activity or commercial gain

If the publication is distributed under the terms of Article $25 \mathrm{fa}$ of the Dutch Copyright Act, indicated by the "Taverne" license above, 


\title{
PRE-RANDOMIZATION IN STUDY DESIGNS
}

\author{
Acceptability and applicability
}

Ron Schellings 
ISBN 9052784809

Lay-out: Thum Aarts

Cover: Audrey Rijcken, oil painting

Printing: Datawyse / Universitaire Pers Maastricht

(C) 2005 R. Schellings

All rights reserved. No part of this thesis may be reproduced or transmitted in any form or by any means, electronic or mechanical, including photocopying, recording or any information storage or retrieval system, without permission in writing from the author, or, when approptiate, from the publishers of the publications. 


\title{
PRE-RANDOMIZATION IN STUDY DESIGNS
}

\author{
Acceptability and applicability
}

\section{PROEFSCHRIFT}

ter verkrijging van de graad van doctor aan de Universiteit Maastricht, op gezag van de Rector Magnificus,

Prof. mr. G.P.M.F. Mols, volgens het besluit van het College van Decanen, in het openbaar te verdedigen op vrijdag 7 oktober 2005 om 14.00 uur

door

Adolf Gertrudis Ludovicus Schellings

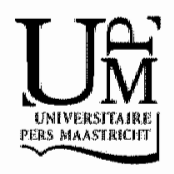




\section{Promotores}

Prof, dr. F. Sturmans

Prof. dr. J.A. Knotmerus

\section{Copromotor}

Dr. G. ter Riet (Academisch Medisch Centrum Amsterdam, Horten Centre Zürich University)

\section{Beoordelingscommissie}

Prof. mr. F.C.B. van Wijmen (voorzitter)

Dr. W.J.M. Dekkers (Radboud Universiteit Nijmegen)

Prof. dr. M.H. Prins

Prof. dr. G.A.M. Widdershoven

Dr. R.A.G. Winkens

In de productiekosten van het proefschrift werd bijgedragen door:

Staatstoezicht op de Gezondheidszorg, Inspectie voor de Gezondheidszorg

Ministerie van VW/S, afdeling Ethiek van de directie Innovatie, Beroepen en Ethiek 


\section{Contents}

1 Introduction

2 Informed consent: an end or a means?

3 Randomized consent designs in randomized controlled trials:

Systematic literature search

4 The Zelen design may be the best choice for a heroin-provision experiment

5 Members of research ethics committees accepted a modification of the randomized consent design

6 Indications and requirements for the use of pre-randomization

7 General discussion

Summary

Samenvatting

Dankwoord

Curriculum Vitae 
$\therefore: 3$ 
1

\section{Introduction}




\section{Background of the thesis}

The use of pre-randomization in srudy designs was first described by Marvin Zelen in 1977. 1 The essence of Zelen's original proposal is that potential participants are randomized before informed consent is obtained." 2 This sequence is contrary to the conventional design in which informed consent is prior to randomization (see Figure 1). The immediate cause for this thesis was a discussion in the mid-Nineties in the Netherlands about the usefulness and ethical acceptability of prerandomization in medical research. That time, a design using pre-randomization (Zelen design) for a heroin provision trial was proposed. , $^{4,5}, 5^{\text {The }}$ use of this design met opposition and disapproval in particular from ethicists and lawyers, but also from physicians and methodologists. ${ }^{6} 10$
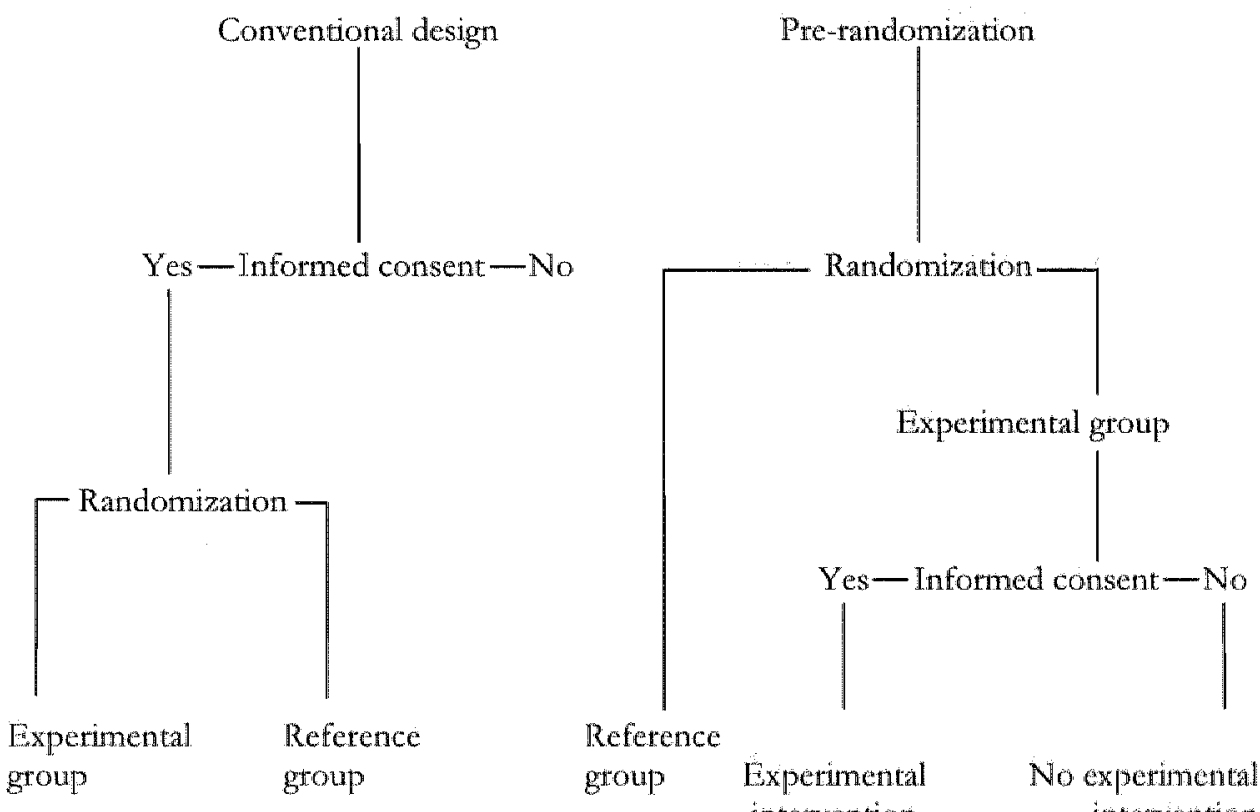

$\begin{array}{ll}\text { Experimental. } & \text { Reference } \\ \text { group } & \text { group }\end{array}$

group Experimental intervention

No experimental intervention

Figure 1. The features of the conventional design in comparison with pre-randomization (Zelen's original proposal).

The most important reasons cited in the literature for rejection of prerandomization are presented in table 1. Finally, the Dutch Parliament agreed upon a medical experiment regarding the effects of heroin provision for long-term heroin addicts conducted according to a conventional study design. Triggered by the conviction that pre-randomization is useful in specific circumstances $11-13$ and against the background that it is often rejected on invalid grounds we started a 
study into the applicability and acceptability of pre-tandomization in study designs. The dissertation approaches the subject of pre-randomization in theory and empinically.

Table 1. Summary of objections in the literature against pre-randomization (in particular the Zelen design).

\begin{tabular}{|c|c|}
\hline & Remarks \\
\hline 1 & Randonization without informed consent is inadmissible \\
\hline 2 & Participantu in reference group are unaware of their participation in a trial \\
\hline 3 & Participation in the reference group is involuntary \\
\hline 4. & Participants in tefertence group cannot withdraw from the trial \\
\hline 5 & Pre randomization does not meet the tequirements of Good Clinical Practice \\
\hline 6 & $\begin{array}{l}\text { Baseline measurements are always necessary, so informed consent is required in all } \\
\text { participants }\end{array}$ \\
\hline 7 & Pre-randomization wiolates the Declaration of Helsinki paragraphs $20-23$ \\
\hline 8 & Pre-randomization is only applicable if other designs are absolutely unfeasible \\
\hline 9 & $\begin{array}{l}\text { Pre-tandomization has newer been used again after Zelen"s introduction in } 1977 \text { and } \\
1979\end{array}$ \\
\hline
\end{tabular}

\section{Variants of pre-randomization}

Marvin Zelen introduced pre-randomization in study designs, that he termed randomized consent designs, to make it easier for physicians to enter patients in randomized clinical trials. Zelen stated that physician reluctance to participate in randomized clinical trials is often a reflection that the physician-patient relationship could be compromised if the physician makes known to the patient his/her inability to sellect a preferred therapy. 1, 2, 14 However, this argument has no methodological importance since it only helps investigators to become less frustrated and could undermine the main purpose of a study design, this is the optimal comparability of the study groups. 'This optimal comparability is achieved only if potential participants, after informed consent, will agree to receive the assigned treatment and the difference in withdrawal of participants between the treatment groups is negligible. If potential participants refuse to contribute to the experimental treatment in Zelen's original proposal there is an apparent loss in statistical efficiency, compared with a conventional design (see appendix).

The interventions in pre-randomization designs can be applied in individuals or on the level of units, or clusters, like for instance primary care practices. ${ }^{11}$ Besides, after randomization, giving information about the intervention, and obtaining informed consent, can be done 1) only in the experimental group (the so called single-consent design) or 2) in the experimental group and in the reference group (the so called double-consent design).

Cluster randomization is a pre-randomization design in which units other than the individual person are randomized and the intervention is at the level of that 
unit or addressed to individuals within that unit. The discussion in the litemture surrounding the ethical, implications of conducting cluster randomized trials is astonishingly thin on the ground, ${ }^{15}, 16$ especially in contrast to pre-randomization designs in which individuals are randomized.

\section{The informed consent paradigm and absolutism}

Conducting scientific research implies a sound informed consent procedure for participants entering a triall. The discussion about the impact of the process of informed consent on patients' autonomy and behavior has been going on. ${ }^{15}, 19-24$ Braunholtz challenges the absolutism of some debaters that informed consent is necessary in all participants independently of the study design by the following example: 25 "at this very moment I might be selecting people on the UK voting register (which is publicly available) to be prayed for (by myself). All the participants are to be tagged, so that I am informed if they die. (This is a public service provided by the registrat for birth and deaths, at a cost). This is surely my affair, and not something I need consent for? But I can just as easily randomize people to be prayed for or not; this is now a randomized trial, but still surely my affair? Suppose I now propose to offer the people for whom I am not praying a free health check. The trial night now be regarded as clinical, and as subject to changes requited by an ethics committee. But the situation has not changed one iota for those in the "prayed-for" arm; so why should my proposals for them be subject to the desires of an ethics committee which might, for instance, insist on consent being obtained for all 'participants"."

This extreme example illustrates the necessity to assess in every study the restriction of the participants' autonomy and the chance of personal ham that could be sustained before and during the trial. Obtaining informed consent is clearly needed in study protocols in which the autonomy of participants is restricted in any way, and/or study maneuvers may cause physical, emotional, and/or social harm.

\section{Legislation and guidelines open to interpretation}

The World Medical Association has developed ethical principles written down in the Declaration of Helsinki, to provide guidance to physicians and other contributors to medical research involving human subjects. The Declaration of Helsinki is the most widely accepted ethical code for medical research. 20 In addition, in most countries, legislation and/or guidelines are promulgated for the application of informed consent procedures in clinical trials. ${ }^{27-29}$ Many lawyers and ethicists interpret these guidelines as prohibiting tesearch with individuals if informed consent or consent by proxy is not or not fully sought. Others accept not obtaining informed consent as conceivable or at least as not explicitly excluded 
by law under very specific conditions. In practice, the decision whether any of these conditions are a valid reason for not (or not fully) obtaining informed. consent, has to be made by a research ethics committee for each trial separately.

\section{Research ethics committees protect study participants' rights}

Nowadays, (bio)medical research involving human subjects must be reviewed by an independent research ethics committee. The need for such committees is grounded in the Sixties and Seventies, ${ }^{30,}, 31$ Although the legislation and guidelines for the composition and method of working of these committees differ substantially between countries, ${ }^{32}$ they serve the same goal, namely to assess both the scientific and ethical aspects of submitted study protocols.

\section{Outline of the thesis}

Chapter 2 is a historical overview of informed consent taking into account that informed consent should be used particularly as a means and not as an end to conduct ethically justifialble research.

Chapter 3 presents a systematic review that describes the use of prerandomization (tandomized consent designs) since the introduction of these designs by Marvin Zelen in 1977.

In chapter 4 we demonstrate the applicability of pre-randomization by means of a fictitious heroin provision experiment.

In chapter 5 we compare the acceptability of a pre-randomization design and a modified form of it by members of medical ethics committees in the United Kingdom and in the Netherlands.

In chapter 6 we describe indications and requirements for the use of prerandomization and we present guidelines for its optimal application.

Finally, chaptet 7 presents a genetal discussion about the usefulness of prerandomization in patient-centered and public health research, considering the consequences of inappropriately designed research for health care and public health. 


\section{References}

1. Zelen M. Statistical options in clinical trials. Semin Oncol 1977, 4.44L6.

2. Zelen M. A new design for randomized clinical trials. N Engl J Med 1979, 300, 12425.

3. Schellings R, Stutmans F. Heröneverstrekking als zorgvermieuwing. Tijoschr Soc Gezondheidsz 1995: 73; 501-2.

4. Schellings $\mathrm{R}$, Kessels AGH, Geurs $\mathbb{R}$, Stumans F. Een herwardering wan het 2 elen design: de bruikbaatheid bij een gerandomiseerd heroine verstrekkingsexperinent. ' $T$ Alc Drugs 1995; 21: 67-78.

5. Schellings R, Kessels AGH, Geurs $\mathbb{R}$, Sturmans F. Overwegingen bij de opzet wan een gerandomiseerd heroine-xerstrekkingsexperiment. Tijdschr Soc Gezondheids 1996 : $74,376-82$.

6. Dute JCJ, Bakker AH. Kroniek gezondheidsrecht: medische experimenten met heroineverstrekking. T Alc Drugs 1995: 21; 108-13.

7. Have H ten. De ethiek van de onderzoeksopzet: gerandomiseerde herome-werstrekking. Tijdschr Soc Gezondheidsz 1996: 74; 374-5.

8. Driessen FMHM, Smeets HM. Zelen design bij experiment heroineverstrekking niet zinvol. T Alc Drugs 1995: 21; 177-8.

9. Roscam Abbing HDC. Toezicht op medisch-wetenschappelijk onderzoek met wilsbekwamen kan niet zonder toetsing. Ned "Tijdschr Geneeskd 1999: 143: 8-10.

10. Gezondheidsraad (Health Council of the Netherlands). Intormed consent en pretandomisatic. Den Haag: Gezondheidsraad, 1999.

11. Knotmerus JA. Gezondheidszorgonderzoek in extramutale settings. In: Ethiek en recht in de gezondheidszorg. Deventer: Kluwer; 1997. p. IV 151-94.

12. Sturmans F. Van wederzijds belang: over nut en noodzaak van academisering van de maatschappelijke gezondheidszorg. Rede, uitgesproken bij de aanvarding van het ambt van bijzonder hoogleraar Epidemiologie en Gezondheidsbeleid aan de Erasmus Universiteit Rotterdam, 1998.

13. Wijmen FCB van, Wijk AS van, Haveman MJ. Nee heb je, ja kun je krijgen: een beschouwing over de toelaatbaarheid van prerandomisatie bij psychiatrisch gezondheidszorgonderzoek. Tijdschr Soc Gezondheidsz 1993: 71; 459-64.

14. Zelen M. Randomized consent designs for clinical trials: an update. Stat Med 1990; 9:6.

15. Edwards SIL, Lilford RJ, Braunholtz DA, Jackson JC, Hewison J, Thornton J. Eithical issues in the design and conduct of randomised controlled trials. Health Technol Assessment 1998; 2 (15).

16. Edwards SJL, Braunholtz DA, Lilford R], Stevens $\Lambda$ J. Ethical issues in the design and conduct of cluster randomised controlled trials. BMJ 1999; 318: $1407-9$.

17. Laforet $G G$. The fiction of informed consent. JAMM 1976; 235: 1579-85.

18. Katz J. Informed consent. Univ Pittsburgh Law Rev 1977; 39: 137-74.

19. Smith R. Informed consent: the intricacies. BMJ 1997; 314: 1059-60.

20. Informed consent in medical research [letters]. BMJ 1997; 314: 14707.83.

21. Informed consent |letters]. BM] 1997; 31.5:247-54.

22. Torgerson DJ, Roland M. What is Zelen's design? BM] 1998; $316: 606$.

23. Smith R. Informed consent: edging forward (and backwards). BMJ 1998; 316:949-51.

24. Stumans F. Informed consent: doel of middel? Tijdschr Soc Gezondheidsz 1997; 75: $114-5$.

25. Braunholz. DA. A note on Zelen randomization: atritudes of parents participating in a neonatal clinical trial. Control Clin Trials 1999; 20:569-72.

26. World Medical Association. Declaration of Helsinki: recommendations guiding physicians in biomedical research involving human subjects. JAMA 1997; 227: 925-6. 
27. Ministry of Hedth, Welfare and Sports. WMO: The Medical Research Involving Humar Subjects Act. The Hague: Ministry of Health, Welfare and Sports, 1998.

28. Medical Research Council Cancer Therapy Conmitte Investigations on human subjects: ethical considerations in the study of cancer therapy. CTG 86/24, February 1987.

29. Final rule-final regulations amending basic HFUS policy for the protection of human rescanch subjects (45 CFR Part 46). Federal Register. January 26, 1981; 46: 8366-91.

30. Godfry E, Wray E, Nicholson R Another look at LREC annual reports. Bull Med Eth $2001 ; 171: 13 \% 21$.

31. Kingma JH, Viersma JW, Wesseling H. Geneesmiddelenonderzoek bij de mens; de gezonde mens als proefpersoon. Ned Tijdschr Geneeskd 1978; 122: 1052-7.

32. Buifsen MAM, Lewwen $\mathrm{E}$ van. De deskundigheid wan medisch-ethische toetsingscommisstes voor mensgebonden onderzoek. Tijdschrift voor Gezondheidsrecht $1999 ; 4: 221-32$. 


\section{Appendix}

Statistical efficiency (power) pre-randomization design (Zelen's oniginal design) wersus the conventional design

\section{Mathematical formulation}

\section{Assumptions:}

- Let $\mathrm{N}$ be the number of eligible participants who are randomly assigned to one of the two treatment groups (both groups have equal size). The reference group (ref) receives standard treatment.

- The proportion of participants that refuse the experimental intervention (exp) is $1-p$ (thus proportion $p$ will participate), they receive standard treatment and will have the same outcome as the reference group.

- All the participants allocated to the reference group will participate in the trial.

- Effect measurements can be obtained for all randomized participants; intention-to-treat analysis is feasible.

- To simplify the formulation it is assumed that the standard deviation of the outcome measure in the experimental group and in the reference group is equal $\left(\sigma_{\exp }=\sigma_{\text {ref }}\right)$.

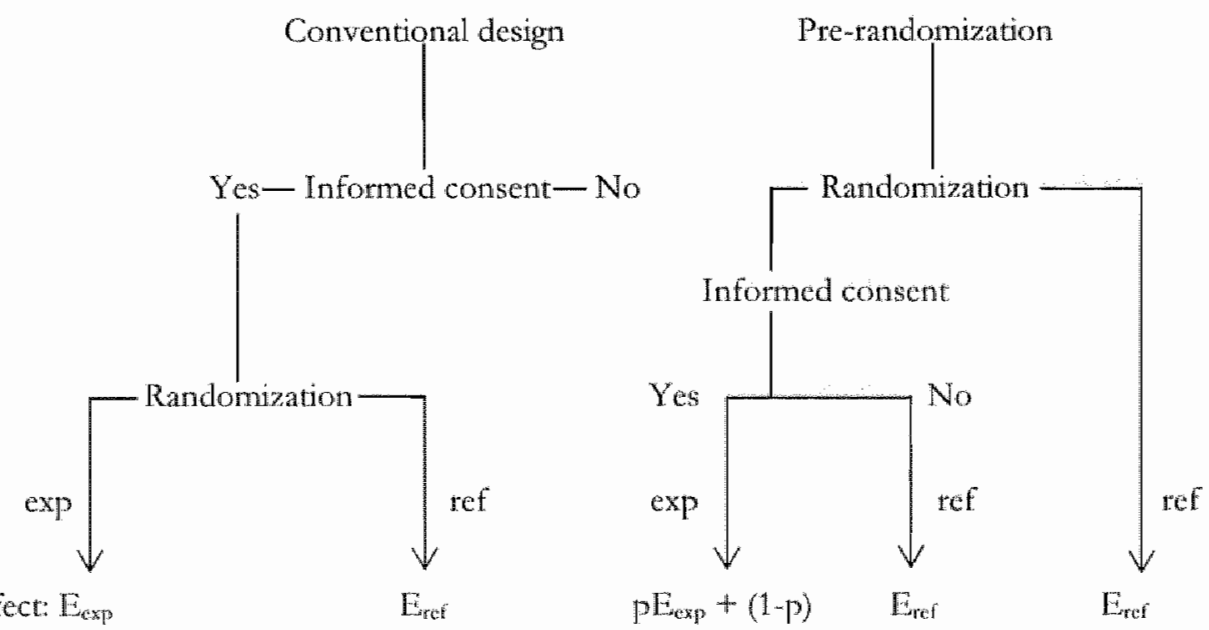


Difference in mean effect between experimental and reference group

$$
\begin{array}{ll}
E_{\text {cxp }}-E_{\text {ref }} & \left(E_{\text {exp }}-E_{\text {ref }}\right)^{*} p \\
\sigma \sqrt{2 /\left(p^{*} \mathrm{~N}\right)} & \sigma \sqrt{2 / N}
\end{array}
$$

Standard deviation of this difference

Test statistic

$$
\frac{\sqrt{p^{*}\left(E_{\exp }-E_{\mathrm{re}}\right)}}{\sigma \sqrt{2 / N}} \quad \frac{p^{*}\left(E_{\text {exp }}-E_{\mathrm{ref}}\right)}{\sigma \sqrt{2 / N}}
$$

Because $p<1$, the test statistic in the pre-randomization design is $\mathrm{Jp}$ smaller than in the conventional design.

This means that in case of a non-compliance rate of, for instance, $25 \%$ in the experimental group in a pre-randomization design, in both study groups $15 \%$ (factor $\sqrt{4 / 3}$ ) more participants is needed to achieve the same power as in a conventional design. 


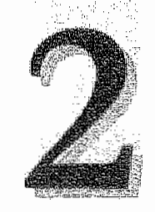

\section{Informed consent: an end or a means?}

Ron Schellings ', Ferd Sturmans ${ }^{2,3}$, Alfons G.H. Kesseis ${ }^{1}$

1 Department of Epidemiology, Maastricht University, the Netherlands

2 Municipal Public Health Service, Rotterdam, the Netherlands

3 Department of Public Health, Erasmus University Rotterdam, the Netherlands

Nederlands Tijdschrift voor Geneeskunde 1999; 143: 1033-1037. (with minor adaptations) 


\section{Abstract}

Since World War II, a variety of protective measures have been introduced for persons receiving medical care or being involved in scientific research. Increasingly, the concept of informed consent has been accepted as a standard.

In experimental therapeutic research, informed consent still causes controversies; individual benefit being weighed against general benefit. In various types of observational research, informed consent also appears to become such an absolute recuirement for many ethicists that observational epidemiological research on the basis of existing data is in danger of becoming impossible.

Both the Declaration of Helsinki and the Personal Registration Act state that, under certain circumstances, it may be possible to deviate from the requirement of obraining individual informed consent. $A$ supervisory committee or a medical ethics committee could then give permission for such a research project.

Informed consent should not be an end in itself, but a means to protect the individual and to guarantee his/her right to autonomy. Insisting on informed consent in situations where autonomy is tespected and no harm or burden is caused, seems to defy the purpose of informed consent. 


\section{Problem Definition}

In the case of interventions that cannot be blinded, it is difficult to design a valid research design. For an experiment with the controlled provision of heroin the socalled pre-randomization or Zelen design was taken into consideration as an attempt to solve this problem. ${ }^{1}$ In this design, the patients are randomized, but only those in the experimental group are asked for their consent; those who refuse their co-operation, receive the regular medical treatment, as do those in the control group. The debate that arose as a result, was almost immediately dominated by earlier statements regarding this research design from both Dutch and foreign literature. The purport of the debate was that this design did not meet the prevailing requirements regarding informed consent. ${ }^{2-4}$ At the same time (the second half of the Eighties), there was a discussion in the British Medical Journal, which was taken up again in 1998, and which resulted in a positive sound from the editor in chief. The latter returned to a certain extent from a point of view taken earlier and stated that the informed consent procedure should not be an end in itself, and we should find a middle course..$^{5,6}$

There is no doubt that informed consent is necessary when study subjects are exposed to (para)medical treatment or when they are prescribed a certain behavior. In the Netherlands, this is regulated by the Medical Research Involving Human Subjects Act (WMO; effective as from mid 1999). ${ }^{7}$ However, if patients in a trial are not approached for, for instance personal measurements, and receive at least the regular care, we believe that informed consent should not be self-evident. In this article, we shall investigate in greater detail the historical arguments concerning informed consent, because we think that in certain situations, the requirement of informed consent defies its purpose.

\section{Right to protection against risks ('primum est non nocere') or the right to autonomy?}

'The first obligation is not to cause damage," is the rule that all physicians consider of paramount importance. It is one of the rules that constitute the basis for medical ethics. Medical intervention is always governed by this principle, both in patient care and later in medical and scientific research. As we will argue later on, this is a rule that - unlike in the past - is increasingly in competition with other medical ethical rules and/or health law, and in particular with the right to autonomy. In the past, medical intervention was hardly the subject of discussion, if at all. The general view was that physicians had the best intentions with their patients. As a result of the great respect that physicians commanded, discussions with patients regarding the preferred treatment were probably rare. In the course of time, however, patients have become more assertive and they no longer accept everything unconditionally. This has introduced in the world of medicine the awareness of the right of self-determination of any human being. Informed 
consent was incteasingly accepted as the standard for medical intervention, albeit not without any problems. The Medical Treatment Agtement Act (WGBO), which states that patients must be informed in detail and should be asked their explicit agreement for all interventions, dates from 1994, but has been implemented gradually in clinical practice. ${ }^{8}$ As was the case in patient care, both in therapeutic and nonmerapeunic research, there was in particular ethical (moral) and legal (judicial) attention for the control of the risks created by the research in question, and not so much on making it possible for the patient to make an autonomous choice. In scientific research, the requirement of informed consent also became mote prominent as the basis of the right to autonomy.

\section{The history of informed consent}

Current views on informed consent in scientific research have been greatly influenced by the excesses of 'scientific research' under the Nazi regime during World Wat II. "The crimes against humanity committed then, have permanenty changed the views on the involvement of human beings in scientific tesearch." During the Nuremberg Trials against the Nazi leaders in 1948 , the court set up guidelines for research, which have become known as the Nuremberg Code. ${ }^{10}$ These were the first written guidelines for scientific research. Core elements are voluntary participation in research and informed consent by study subjects. However, this code proved too restricted and was soon felt to be inadequate. The proposals for improvements eventually led to the Declaration of Helsinki, which was drawn up in 1964.11,12 In this declaration, informed consent also plays a key rolle. The need for informed consent was linked to the nature of the scilentific research. For this purpose, an important distinction was introduced: therapeutic research (research combined with patient care) and non-therapeutic research. The Declaration of Helsinki demands informed consent in all cases of non-therapeutic resentch.

On the other hand, informed consent is apparendy not always required in therapeutic research. If physicians deem it of vital importance not to ask for informed consent, then specific reasons for such a proposal must be included in the protocol that is submitted to the research ethics committee. This exception remains in later versions of the Declatation. In spite of its shortcomings, the Declaration of Helsinki is regarded as the core document in the history of research cthics.

The Nuremberg Code was the first code written by any legal system for the field of medicine; the Declatation of Helsinki is a code writtem by and for professional colleagues. Stimulated by this development, individual scientists statted to publish articles. Beecher was one of the first and most prominent ones. ${ }^{13}$.15hough he emplhasized the need for informed consent, he also noted that thete are different forms of informing for different types of research. Beecher 
was not an advocate of any approach to supervision of experiments that was based on absolute rules. He believed that, however necessary rules and regulations may sometimes be, they generally did more bad than good. Unlike Beecher, the Briton; Papworth, was an avid proponent of principles and tights. 16 It was not clear; however, whether these were regarded as the right to autonomy of the right to protection against risks, or both.

In 1972, an anthology by Katz er al ${ }^{17}$ appeared. This publication saw the light on the eve of the disclosure of what may have become the most notorious example of abuse in research in the United States of America: the Tuskegee syphilis experiment. In this experiment, parients - without being told - were involved in a research project in which the natural course of syphilis was 'merely' observed. Even when it had become known that penicillin was effective against syphilis, patients were denied this knowledge and did not receive this medication. ${ }^{\text {is }}$ "This event, in addition to several others that were in violation of the principle of informed consent, led to the discussion on the position of codes in the regulation of biomedical research on humans being brought out into the open, ${ }^{19-21}$ "This was the start of a complex interaction between scientific publications, journalism, public indignation and legislation. ${ }^{9}$

\section{Informed consent in behavioral science}

The first draft code that discussed ethical standards in behaviorall research appeared in 1951.22 It mentioned neither consent, nor informed consent. Under the heading of 'protection of the well-being of study subjects,' the following proposal was made: "Only if the problem to be studied is important and it cannot be studied in any other way, the psychologist may withhold information, or temporarily give misinformation to study subjects, or expose them to emotional stress. If there is any danger of any serious after-effects, the research can only be done after the study subjects have been fully informed with respect to this sisk and only then sign up as volunteers." Any attempt to develop principles for research in psychology must therefore be reconciled with the use of 'deception' as a research method.

During the Sixties, "deception' became the topic of a major moral controversy within the field of psychology. As a result, the interest that had so far only been directed at the ethics of clinical practice was extended in the direction of research ethics. In the Seventies, this led to the creation of a special code, aimed exclusively at research involving human study subjects. In 1966, the "Wichita Jury Stady", ${ }_{2}^{23}$ in which professors of sociology and law from the University of Chicago studied the deliberations of six different juries without the latter being aware of this, was the first situation in the psychology literature in which the opponents of this type of research in behavioral science emphatically defended the requirement of informed consent on the basis of the autonomy principle. The original researchets, however, 
referted to the concept of general welfare and in particulat the principle of beneficence: in the interest of general welfare it was intolerable that there were no empirical data and that we were ignorant of what really happened in such a fundamental institution as jury law.

In 1972 , the Cook Commission presented a draft behavional code. ${ }^{24}$ This also allowed for a deviation from the ptinciple of informed consent and explicitly left room for the possibility of using deception as a research instrument. Although it was considered unethical to involve indrviduals in research without their knowledge and informed consent, exceptions should remain possible. In particular if (a) it could be shown that the research objectives could not be achieved without using deception; (b) there was sufficient reason for using deception, as shown by the fact that it could be expected that the study subjects would consider this reasonable if they were later informed about the rationale; (c) the researcher would take full responsibility for detecting and eliminating stressful after-effects; and (d) the study subjects would eventually benefit from the research experience. During the period of 1974-1983, two more commissions were created who were to concentrate both on medical and biomedical research, and on behavioral research: these wete the National Commission for the Protection of Human Subjects in Biomedical and Behavioral Research and the President's Commission for the Study of Ethical Problems in Medicine and Biomedical and Behavioral Research. ${ }^{25}$ Both commissions aimed at a uniform code for all types of research, including observational reseatch.

\section{Informed consent in therapeutic research}

In therapeutic research, there is the development of using single- and double-blind, randomized experiments for the evaluation of new treatments. The double-blind randomized experiment is increasingly becoming accepted as the gold standard for evaluating new treatments. This has also led to greater need to protect patients against obtusive physicians, who may favor the interests of future patients over those of the present ones when they weigh these interests against one anothet. After all, carrying out a scientific medical experiment implies dropping the current treatment, which may or may not have shown its menit beyond most experts" doubt and to replace it by a new treatment, in order to test the value of that new teatment (the value of which has not been convincingly shown yet). As the researcher affects both the experimental group and the reference group (which may or may not receive a placebo or sham treatment), this requires the informed consent of all participants. The study subjects must be aware of the research conditions, possible side effects et cetera. The basic human rights, and in particular the right to autonomy, ${ }^{26}, 27$ do not allow the interest of the individual to be sacrificed for the interest of society, or the advancement of science, or the scientist. 
Baum on the other hand, ${ }^{28}$ has completely different views. He suggests that patients have a moral obligation to participate in scientific research. He argues in favor of a broadly based support for research, by mobilizing public opinion, informing risk groups, and pointing out the importance of scientific tesearch.

In the Netherlands, protection of the interests of the individual is laid down in the Person Registration Act. ${ }^{29}$ Prompted by the investigation of falling heart valves, Vandenbroucke and Grobbee published a concerned comment: they fear that the framework of this act will also make informed consent an absolute requirement for observational research, to the extent that in particular epidemiological research on the basis of existing data will become impossible. ${ }^{30}$ tit would no longer be possible to observe and register the behavior of an individual, unless the person involved were first explicitly asked whether observation and registration was allowed. This introduces the so-called. Hawthome effect and carries with it the risk of possible bias. ${ }^{31}$ (This effect consists of the research results being biased because the study subjects know that they are being observed). A possible way out would be offered by stating in the privacy regulations that if scientific research makes use of private data, this requires the permission of a superwisory committee with respect to the registration of individuals. The Medical Research Involving Human Subjects Act (WMO) is almed at protecting study subjects if such individuals are likely to be burdened by the research. ${ }^{7}$

In most studies, it is necessary to compare the results with data from an 'external' reference group. This involves individuals who have not participated in the experiment, who have no knowledge of the study, who experience no inconvenience or discomfort, and who suffer no damage with regard to their therapy because they receive the same treatment that they would always have had. This is the case, for example, when Zelen's pre-randomization design is used. ${ }^{32}$ Nevertheless, this type of research is the subject of discussion, because the 'owner' of the data to be analyzed has not given permission for the analysis, and hence it is not allowed. ${ }^{33}$

We therefore wonder whether ethicists who specialize in medical therapeutic research should not start a dialogue with their colleagues in the behavioral sciences, in order to prevent that medical therapeutic researchers are forced to apply all kinds of artificial maneuvers.

\section{Conclusion}

The principle of not causing damage and the principle of the right of autonomy have, cuite rightly, led to a variety of protecrive measures for the individual: active protection in the form of informed consent, and passive protection in the form of the ban on the use of data without permission. Informed consent is an autonomous authorization by a patient or study subject, with the aim of guaranteeing these rights. The prevailing legislation and regulations in the 
Netherlands provide adequate protection of the tights of patients involved in scientific research.

Under certain circumstances, however, we believe that beneficence and justice offer sufficient reason for giving up the right to absolute autonomy. To live in a sociery where autonomy is the only main goal cannot be the ultimate principle, as Callahan stated. ${ }^{34}$ Warnock would prefer to change the term "principle of autonomy' into 'principle of non-exploitation", ${ }^{35}$ because the key issue is to prevent the possible exploitation of study subjects. Research, including clinical research, is after all not aimed at the welfare of just some individual patients, but at the production of medical knowledge that benefits society as a whole. The individual patient could only benefit by accident. In a research program, study subjects are used as a means; they do not constitute the goal. This is not morally teprehensible if individuals offer their services voluntarily. According to Warnock, there is a great difference between, on the one hand, the situation in which anonymous data, collected in a particular study, are used for a different problem that had not been studied before, and on the other hand, the situation in which drugs for a disease are tested in a randomized trial. In the first case, he argues that there is no damage to the study subjects and the term 'exploitation' would be an exaggeration. It is therefore incorrect to state that not obtaining informed consent in itself causes damage. Sometimes this means exploitation, at other times it does not. Informed consent was never meant as an end in itself, but as a means to obtain protection for the individual and guaranteeing his/her right to autonomy. In those cases in which this right has very little meaning, because there is no possibility of damage or burden, the unconditional requirement of informed consent appears to have missed the mark. That is when informed consent has become an end tather than the means. 


\section{References}

1. Stumans F. Van wederzijds belang: over nut en noodzalk wan academisemg wh de maatschappelike gezondheidszorg [naugurele rede]: Erasmus Universitet Rotrendam; 1998.

2. Ellenberg SS. Randomization designs in comparative clinical trals. N Engl I Med 1984; 310: $1404-8$

3. Bergkamp L. Het proefdier mens. De nometing en regulering van medische experimenten met mensen. Alphen aan den Rijn: Samson; 1988.

4. Beaufort ID de, Mulder JH. Informed consent en experimenten met mensen. 'Zelen revisited" Informed consent en prenandomisatie. Metamedica 1987; 66:454-60.

5. Smith R. Commentary: The importance of patients" consent for publication. BM] 1996; 313: 16 .

6. Smith R. Informed consent: edging forwards (and backwards). BMJ 1998; $316: 949.51$.

7. Ministry of Health, Welfare and Sports. WMO: The Medical Research Involving Human Subjects Act. The Hague: SDU; 1996.

8. Wet Geneeskundige Behandelingsovereenkomst, Staatsblad 1994: 837. 's-Gravenhage: Staatsuitgeverij; 1994.

9. Faden RR, Beauchamp TH, King NMP. A history and theory of informed consent. New York: Oxford University Press; 1986.

10. Trials of war ctiminals before the Nuremberg military tribunals under Control Council Law nr 10: 181-2. unal I 1947. Washington D.C.: US Govemment Printing Office; 1949.

11. World Medical Association. Declaration of Helsinki: recommendations guiding physicians in biomedical research involving human subjects. JAMA 1997; 227: 925-6.

12. Aanscherping van de ethische criteria woor medisch-wetenschappelijke verslaglegging en de Verklaring van Helsinki. Ned Tijdschr Geneeskd 1999;143: 33-6.

13. Beecher HK. Experimentation in man. Springfield, Ill : Thomas; 1959.

14. Beecher HK. Ethics and clinical research. N Engl J Med 1966; 274: 1354-60.

15. Beecher HK. Consent in clinical experimentation: myth and reality. JAMA 1966; 195: 34-5.

16. Papworth MH. Human guina pigs: Experimentation on man. Boston: Beacon Press; 1967.

17. Katz J, Capran $A$, Swift Glass E. Experimentation with human beings. New York: Russel Sage Foundation; 1972.

18. Brandt A.M. Racism and research: The case of the Tuskegee syphilis study. Hastings Cent Rep 1978; 6: 21-9.

19. Levine R]. Ethics and regulation of clinical research. Baltimore: Urban \& Schwarzenberg: 1981. p 51-3.

20. Ingelfinger IJ. Ethics of experiments on children. N Eingl J Med 1973; 288:791-2.

21. Ward R, Krugman S, Giles JP, Jacobs AM, Bodansky O. Infectious hepatits: studies of its natural history and prevention. $\mathrm{N}$ Engl J Med 1958; 258: 407-16.

22. APA Committee on Ethical Standards for Psychology. Ethical standards for psychology. Am Psychol 1951; 6: 427-52.

23. Ruebhausen OM, Brim OG. Privacy and behavioral research. An Psychol 1966;21: 423-37.

24. American Psychological Association (APA). Ethical principles in the conduct of research with human participants. Washington: APA; 1973. 
25. Pxesident Commission for the Study of Ethical Problems in Medicine and Biomedical and Behavioral Research. Making health care decisions: the ethical and legal implementawons of informed consent in the patient-practioner relationship. Washington, D. C. US Government Printing Office; 1982.

26. Doyal L. Informed consent - a response to recent correspondence. BM] 1998 ; 316 : 10001 .

27. Have H ten De ethiek wan de onderzoelksopzet: gerandomiseetde heroineverstrekking. Tildschr Soc Gezondheidszorg $1996 ; 74: 374-5$.

28. Baum, M. Mew approach for tecruitment into randomized controlled trials. Lancet $1993,341,812-3$

29. Wet persoonsregtstraties. Staatsblad 1988: 665."S-Gravenhage: Staatsuitgeverij; 1989.

30. Vandenbroucke JP, Grobbee DE. Lessen uit een kunsthartklep-controwerse. Ned Tijdschr Geneeskd 1998; 142: 1648-52.

31. Bouter IM, Dongen MCJM van. Epidemiologisch onderzoek: opzet en interpretatie. Houten: Bohn Stafleu Van Loghum; 1995.

32. Zelen MA. A new design for randomized clinical trals. N Engl J Med 1979; 300: 12425.

33. Beraadsgroep 'Gezondheidsethick en Gezondheidstecht'. Privacy bij postmatketing surveillance. Den Haag: Gezondheidsrad; 1993. p 27-9.

34. Callahan D. Autonomy: a moral good, not a moral obsession. Hastings Center Repott 1984, 11: 40-2.

35. Warnock M. Informed consent - a publisher's duty. BMJ 1998; 316:1002-3. 


\section{Randomized consent designs in randomized controlled trials: Systematic literature search}

Ron Schel]ings ${ }^{1}$, Alfons G.H. Kessels ${ }^{2}$, Gerben ter Riet ${ }^{3,4}$, J André Knottnerus, Ferd Sturmans

1 Public Health Supervisory Service of the Netherllands, the Health Care Inspectorate, The Hague, the Netherlands

2 Department of Clinical Epidemiology and Medical Technology Assessment, University hospital Maastricht, the Netherlands

3 Academic Medical Center, Department of General Practice, Amsterdam, the Netherlands

4 Horten Centre, University of Zürich, Switzerland

5 Department of General Practice, Maastricht University, the Netherlands

6 Department of Public Health, Erasmus University Rotterdam, the Netherlands. 


\section{Abstract}

Background Three types of randomized consent designs are distinguished, ranked according to the extent to which participants are informed about treatment options: single-consent (those in the experimental group learn about their assigned treatment), incomplete-double-consent (all participants learn about their assigned treatment), and complete-double-consent (all participants learn about all treatnents studied). All are methodologically, ethically, and judicially controversial. Even so, their use is justified if blinding is deemed necessary, but impossible to achieve by sham procedures (placebo), and experimental treatment seems attractive to potential participants.

Objective To give a comprehensive overview of the use of randomized consent designs.

Data sources MEDLINE (1/1977-2/2003), EMBASE (1/1984-2/2003), PsycINFO (1/1996-2/2003), the Cochrane Library, and the Science Citation Index database.

Review methods Eligible were studies using a fandomized consent design. Cluster randomized trials were excluded. One teviewer selected and data-extracted eligible papers. A second reviewer independently data-extracted $10 \%$ of the papers. Data on country of study conduct, year of commencement, area of medicine, type of design, reason(s) for use, details on approval by a research ethics committee, the index and reference intervention, nature of endpoints, and details on collection of data were extracted. Furthermore, for each trial, the rates of non-compliance and loss to follow-up were registered by treatment arm. The three types of randomized consent designs were compared as to differences between the rates of noncompliance and loss to follow-up in the separate trial arms.

Results Randomized consent designs are seldom used $(n=50)$. When used, they have often been used in the wrong circumstances (misuse). In $65 \%$ of the studies the non-compliance in the index group is larger than in the reference group. Contrary to expectation, trials using the incomplete-double design were associated with significantly higher rates of non-compliance and loss to follow-up in the reference groups than trials employing the other two versions.

Conclusion Trialists and physicians should be aware of the proper indication for the use of randomized consent designs. 


\section{Introduction}

In 1977, Marvin Zelen proposed the randomized single-consent design, which he elaborated upon in 1979.1. 2 The key characteristic of this design is that, bofore informed consent is sought, patients are randomized to an index group or to a reference group. The patients in the index group are approached for informed consent. The patients randomized to the reference group are not approached for informed consent. However, they are given the best standard treatment. Any index patient that declines is also offered best standard treatment. The reason to introduce this design was to avoid problems associated with persuading physicians to enter patients into clinical trials. ${ }^{2}$ In particular, the design was meant to circumvent difficulties with the randomization procedure and obtaining informed consent for participation. ${ }^{3}$

The randomized single-consent design evoked a wave of criticism from many ethicists and health lawyers, who argued that the participants in the reference group are not even aware of their involvement in a study. ${ }^{47}$ From an ethical point of view, informed consent should be sought from all study participants after an explanation of the purpose of the study, the main features of the assigned and the alternative study $\operatorname{arm}(\mathrm{s})$, and effect measurements. The opposition to the randomized single-consent design is reflected in national regulations. For example, since the $1980 \mathrm{~s}$, the design is not acceptable to the British Medical Research Council ${ }^{8}$ and to U.S. federal research institutes. ${ }^{9}$ To overcome ethical concerns, the randomized double-consent design as a counterpart of Zelen's single-consent design has been introduced. ${ }^{3},{ }^{10-12}$ In this design, informed consent is sought in all treatment arms after randomization.

A serious methodological drawback of the randomized single-consent clesign is the large potential for bias due to post-randomization withdrawal from the index group after the informed consent procedure. ${ }^{13-14}$ Since the randomized doubleconsent design gives randomized patients in all treatment arms an opportunity to refuse their allocated treatment, and opt for alternatives, it is associated with an even greater likelihood of participants' crossing over to other treatment arms. This, in turn, increases the likelihood and the magnitucle of bias in the estimate of the true treatment effect as compared to the single-consent design. To minimize the occurrence of this bias, a version of the double-consent design has been used in which participants in the reference group are informed about their own treatment only, but in which information about the alternative treatment is withheld. ${ }^{3}{ }^{10-12}$ Further details on why many ethicists think that randomized consent designs are problematic may be found in references $15-18$.

In our opinion the use of a randomized consent design should be considered. only when blinding with a placebo or sham procedure is impossible, combined with at least one, to some extent, attractive treatment option that participants could easily acquire and implement themselves, such as an over-the-counter drug. ${ }^{1 \%, 20}$ 
The main purpose of the present paper is to review systematically all randomized studies using a randomized single or double-consent design and to determine how they wete used, the cited reason(s) for their use, and how often reference was made to approval by research ethics committees. To describe the extent to which the use of these designs was differentially successful, we also studied the tates of non compliance and loss to follow-up, separately for each trial arm. Cluster-randomized trials are also applications of the randomized consent design. However, these are beyond the scope of this review. Also, this is a review of the experience of using randomized consent designs, not a review of the ethical litefaturc on the merits of the design.

\section{Methods}

\section{Searches and eligibility}

The eligibility criterion was trials in which any type of randomized consent design was used. We searched MEDLINE (Index Medicus 1/1977-2/2003), EMBASE (Fixcerpta Medica 1/1984-2/2003), PsycINFO (1/1996-2/2003) and the Cochrane Library (2002, issue 2) and the Science Citation Index database (Web of Science by Institute for Scientific Information). The latter was seatched using the cited reforence seards option on reference 1,2 and 12 . There were no language restrictions. The search terms used were: Zelen design, pre-randomiz(s)ation, prerandomiz(s)ation, randomiz(s)ed consent design, postrandomiz(s)ation consent, postrandomiz(s)ation design, preconsent randomiz(s)ation, preconsent desnign, randomiz(s)ed single(-)consent design, randomiz(s)ed double(-)consent design (spelling alternatives are in brackets). We selected studies in which a randomized consent design was used. Any discussion papers and papers focusing on methodological or statistical issues related to a randomized consent design were used as background literature. Titles and abstracts found by the electronic searches were scrutinzed by RS. Refenence lists of all eligible papers were checked including those of background papers. The selected trials were included in the review if a randomized consent design had been used. Selection and exclusion of full papers was done by RS.

\section{Data extraction}

Study chatacteristics were extracted from the included papers by RS and, as a pilot, a $10 \%$ random sample was sctutinized independently by GtR. There were only minor disagreements between the reviewers. Study characteristics were easily resoluble and the level of agreement justified the other $90 \%$ of data extraction. Study chatacteristics that were extracted included country of study conduct, year of commencement, area of medicine, exact type of randomized consent design, cited reason(s) for use of this design, details on approval by a research or medical ethics 
committee, the index intervention, reference intervention, nature of endpoints (medical us behavioral), and details on how and when during the trial data were collected. Furthermore, for each trial, the rates of non-compliance and loss to follow-up were registered by treatment arm. We defined non-rompliance as any violation of the procedures described in the study protocol, for instance the rejection of the allocated treatment (allowing measurement of the outcome, however), the acquisition of additional treatment, or switching from one treatment (arm) to the other (contamination). Non-compliance may reduce the validity of $n$ trial and, in an intention-to-treat analysis, may alter the intended treatment contrast. Loss to follow-up was defined as a failure to acquire outcome measurements irrespective of the refusal of treatment allocation. It is indicated by missing data on end points and, as a consequence, these patients cannot be included in the statistical analysis of outcome, unless a ((few) well chosen) value(s) for this measurement is imputed. ${ }^{21} \mathrm{~A}$ study's internal validity is reduced if the patients who are lost to follow-up are prognostically different from the patients who complete the study and the loss to follow-up rates differ between the treatment groups. We analyzed the differences in non-compliance rates and loss to follow-up rates berween double-consent designs in which participants received full information on treatment alternatives (the complete-double-consent design), double-consent designs in which information on (the existence of) the alternatives was witheld (the incomplete-double-consent design), and the single-consent design. We expected that the complete-double-consent design, in particular, would be vulnerable to both of these threats to internal validity.

\section{Statistical Analysis}

Comparisons of proportions between groups were analyzed with the KruskalWallis test or the Mann-Whitney $U$ test. To test correllation the Spearman rank correlation coefficient was used. To test null hypotheses a significance level of 0.05 (two-sided) was used.

\section{Results}

The electronic searches yielded 630 papers and we obtained full papers of 114 . Of these, 60 concemed trials in which a randomized consent design appeared to have been used, and 54 papers were discussion papers, and papers focusing on methodological or statistical issues. The reference lists of the latter revealed six additional trials. Thus, initially, 66 papers were included in the review. $\Lambda$ fter a more detailed analysis, it appeared that eight trials had not used a randomized consent design and these were excluded Thus, 58 papers, representing 50 trials, were formally data-extracted. Six trials were published by more than one paper and dataextraction was done on the most recent paper (Table 1). 
Table 1 . The resules of the systematic search.

\begin{tabular}{llc}
\hline Stage & Action & No of citations selected \\
\hline 1 & Search & 630 \\
2 & Duplicates and other topics removed & 114 \\
3 & Exclusion of non-randomized consent designs & 66 \\
4 & Exclusion on basis of full text & 58 \\
5 & Exclusion papers presenting the same study & 50 \\
\hline
\end{tabular}

\section{Reasons for use}

The single-consent design was used in 23 trials. Twenty-seven used the doubleconsent design (Table 2). Of the latter, two began as single-consent studies, but switched to a double-consent design. One trial started using double-consent, but switched to a conventional design in which consent is sought before randomization. In four of the 27 double-consent design trials, all participants were fully informed. In 23, information about the alternative treatment was withheld in the reference group.

In 29 of the 50 trials, the reason(s) for using a randomized consent design was/were given. The prevention of contamination as the main reason was cited 8 times in the 23 single-consent, and 8 times in the 27 double-consent designs. Eleven papers cited avoiding problems with randomization as the main reason (seven times in single-consent designs; four times in double-consent designs), including a simpler informed consent procedure, simpler participant recruitment, and avoiding unnecessary confusion and distress for patients, which were thought to be associated with the request for consent. In two of the 17 studies, where baseline data were collected through measurements necessitating personal contact with participants, a single consent design was nevertheless used for reason of avoiding contamination. In one psychiatric study it is unclear whether the participants were able to give consent. ${ }^{63}$ Of the trials started before 1986,48 percent mentioned approval by a research ethics committee. This number was 85 percent among trials statted in 1986 or after. It is unclear why the other $15 \%$ of trials were published without mentioning such approval. 


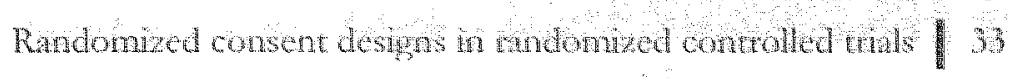

Table 2 see next page 
Table 2. Study claracteristics of 50 randomized single-consent and double consent

\begin{tabular}{|c|c|c|c|c|c|}
\hline $\begin{array}{l}1^{\text {ti author wet }} \\
\text { (country, year) }\end{array}$ & $\begin{array}{l}\text { Year } \\
\text { begun }\end{array}$ & $\begin{array}{l}\text { Area of } \\
\text { medicine }\end{array}$ & Disorder & Design & $\begin{array}{l}\text { Main reason } \\
\text { use design }\end{array}$ \\
\hline $\begin{array}{l}\text { Moertel 2z } \\
\left(U_{S}, 1984\right)\end{array}$ & 1965 & Oncology & $\begin{array}{l}\text { Gastric } \\
\text { carcinoma }\end{array}$ & Single & Cont \\
\hline $\begin{array}{l}\text { Fisher } 2 x_{2} 24,25 \\
\text { (US, 2002) }\end{array}$ & 1976 & Oncology & Breast cancer & Double & Cont \\
\hline $\begin{array}{l}\text { Fisher } 26 \\
(U S, 1988)\end{array}$ & 1.977 & Oncology & Rectal cancer & Double & Rand \\
\hline $\begin{array}{l}\text { Wolmark } 27 \\
\text { (US 1988) }\end{array}$ & 1977 & Oncology & Colon cancer & Double & - \\
\hline $\begin{array}{l}\text { Santen, Wells, } 20,29 \\
(\mathrm{US}, 1981,1982)\end{array}$ & $<1978$ & Oncology & Breast cancer & Double & - \\
\hline $\begin{array}{l}\text { Lange }{ }^{30} \\
\text { (US 1984) }\end{array}$ & 1980 & Oncology & Leukemia & Single & Cont \\
\hline $\begin{array}{l}\text { Andersson }{ }^{31} \\
\text { (Denmark, 1999) }\end{array}$ & 1980 & Oncology & Breast cancer & Double & - \\
\hline $\begin{array}{l}\text { Mansout }{ }^{32} \\
\text { (US 1989) }\end{array}$ & 1981 & Oncology & Breast cancer & $\begin{array}{l}\text { Double- } \\
\text { conventional }\end{array}$ & w \\
\hline $\begin{array}{l}\text { Hardcastle }^{33} \\
(\mathrm{UK}, 1996)\end{array}$ & 1981 & $\begin{array}{l}\text { Oncollogy } \\
\text { screening }\end{array}$ & Colorectal cancer & Single & - \\
\hline $\begin{array}{l}\text { Pearl O'Rourke }{ }^{34} \\
\text { (US, 1989) }\end{array}$ & 1982 & Pediatrics & $\begin{array}{l}\text { Pulmonary } \\
\text { hypertension }\end{array}$ & Single & Rand \\
\hline $\begin{array}{l}\text { Bartlett }{ }^{35} \\
(\text { US, 1985) }\end{array}$ & 1982 & Pediatrics & $\begin{array}{l}\text { Respiratory } \\
\text { failure }\end{array}$ & Single & Rand \\
\hline $\begin{array}{l}\text { Hojnis } 36,37,38 \\
\text { (Denmark, 1999) }\end{array}$ & 1982 & Oncology & Breast cancer & Double & - \\
\hline $\begin{array}{l}\text { Balch }{ }^{34} \\
(\text { US, 2001) }\end{array}$ & 1983 & Oncology & $\begin{array}{l}\text { 1-4 mm } \\
\text { Melanomas }\end{array}$ & Double & - \\
\hline $\begin{array}{l}\text { Blichert-Toft } 40.41 \\
\text { (Denmark, 1992) }\end{array}$ & 1983 & Oncology & Breast cancer & $\begin{array}{l}\text { Single- } \\
\text { double }\end{array}$ & Other \\
\hline
\end{tabular}

\footnotetext{
* studies with no index groups and one reference group.

† Cont: to avoid contmination; Rand: to avoid problems with randomization; Other: other teason.

* no information given in the rewiewed paper.

5: Rata collected routinely or by means of a register; $P$ : Data (also) collected by personal contact with participants.
} 
designs in different areas of medicine.

\begin{tabular}{|c|c|c|c|}
\hline $\begin{array}{l}\text { Approved by } \\
\text { ethics } \\
\text { committee }\end{array}$ & Intervention & Reference & $\begin{array}{l}\text { Baseline / } \\
\text { effect } \\
\text { registration }\end{array}$ \\
\hline$-t$ & 5-fluorouracil \& radiation & Observation & $\mathrm{R} / \mathrm{R}$ \\
\hline - & $\begin{array}{l}\text { Lumpectomy } \\
\text { Lumpectomy \& irradiation }\end{array}$ & Mastectomy & $\mathrm{R} / \mathrm{P}$ \\
\hline - & $\begin{array}{l}\text { Chemotherapy } \\
\text { Radiation therapy }\end{array}$ & Observation & $\mathrm{R} / \mathrm{R}$ \\
\hline - & $\begin{array}{l}\text { Chemotherapy } \\
\mathbb{B C G} \text { administration }\end{array}$ & Observation & $\mathrm{R} / \mathrm{R}$ \\
\hline - & $\begin{array}{l}\text { Aminoglutethimide \& } \\
\text { hydrocorüsone }\end{array}$ & Surgical adrenalectomy & $\mathrm{R} / \mathrm{R}$ \\
\hline Yes & $\begin{array}{l}\text { Trimethoprim-sulfa- } \\
\text { methoxazole \& nystatin }\end{array}$ & No prophylaxis & $\mathbb{R} / \mathbb{R}$ \\
\hline - & $\begin{array}{l}\text { Chemotherapy } \\
\text { (CAF \& TAM) }\end{array}$ & $\begin{array}{l}\text { Chemotherapy } \\
\text { (CMF \& TAM) }\end{array}$ & $\mathbb{R} / \mathbb{R}$ \\
\hline- & Chemotherapy & Observation & $\mathbb{R} / \mathbb{R}$ \\
\hline Yes & Weacal-occult-blood test & No intervention & $\mathrm{R} / \mathrm{R}$ \\
\hline Yes & $\begin{array}{l}\text { Extracorporeal Membrane } \\
\text { Oxygenation }\end{array}$ & $\begin{array}{l}\text { Conventional medical } \\
\text { therapy }\end{array}$ & $\mathrm{R} / \mathrm{R}$ \\
\hline Yes & $\begin{array}{l}\text { Extracorporeal Membrane } \\
\text { Oxygenation }\end{array}$ & Conventional therapy & $\mathrm{R} / \mathrm{R}$ \\
\hline Yes & $\begin{array}{l}\text { Chemotherapy \& } \\
\text { radiotherapy }\end{array}$ & Chemotherapy & $\mathrm{R} / \mathrm{R}$ \\
\hline- & $4 \mathrm{~cm}$ radial excision & $2 \mathrm{~cm}$ radial excision & $\mathrm{R} / \mathrm{R}$ \\
\hline Yes & Breast preserving therapy & Mastectomy & $\mathrm{R} / \mathrm{R}$ \\
\hline
\end{tabular}


Table 2. Contunued.

\begin{tabular}{|c|c|c|c|c|c|}
\hline $\begin{array}{l}1^{\text {wit athor }} \text { authof } \\
\text { (country, year) }\end{array}$ & $\begin{array}{l}\text { Year } \\
\text { begun }\end{array}$ & $\begin{array}{l}\text { Area of } \\
\text { medicine }\end{array}$ & Disorder & Design & $\begin{array}{l}\text { Main reason } \\
\text { use design }\end{array}$ \\
\hline $\begin{array}{l}\text { Sell }{ }^{42} \\
\text { (Denmark, 1991) }\end{array}$ & 1983 & Oncology & Bladder cancer & Single & - \\
\hline $\begin{array}{l}\text { Fline }{ }^{43} \\
(\mathrm{UK}, 1989)\end{array}$ & 1983 & Midwifery & $\begin{array}{l}\text { Low obsterric } \\
\text { risk }\end{array}$ & Double & \\
\hline $\begin{array}{l}\text { Ganz }{ }^{4} \\
\text { (US, 1988) }\end{array}$ & 1984 & Oncology & Lung cancer & Double & - \\
\hline $\begin{array}{l}\text { Hemminki } 45 \\
\text { (Finland, 1989) }\end{array}$ & 1985 & Public health & $\begin{array}{l}\text { None } \\
\text { (pregnancy) }\end{array}$ & Double & Rand \\
\hline $\begin{array}{l}\text { Kronborg } 46 \\
\text { (Denmark, 1996) }\end{array}$ & 1985 & $\begin{array}{l}\text { Oncology } \\
\text { screening }\end{array}$ & $\begin{array}{l}\text { Colorectal } \\
\text { cancer }\end{array}$ & Single & Cont \\
\hline $\begin{array}{l}\text { Riethmülller }{ }^{47} \\
\text { (Germany, 1994) }\end{array}$ & 1985 & Oncology & $\begin{array}{l}\text { Colotectal } \\
\text { carcinoma }\end{array}$ & Single & Cont \\
\hline $\begin{array}{l}\text { Korvick }{ }^{48} \\
\text { (UK, 1992) }\end{array}$ & 1985 & $\begin{array}{l}\text { Internal } \\
\text { medicine }\end{array}$ & $\begin{array}{l}\text { Pseudomonas } \\
\text { Aeruginosa }\end{array}$ & Single & Rand \\
\hline $\begin{array}{l}\text { Bryce } 49 \\
\text { (Australia, 1991) }\end{array}$ & 1985 & Obstetrics & Preterm birth & Single & Cont \\
\hline $\begin{array}{l}\text { Lambe so } \\
\text { (US, 1986) }\end{array}$ & - & $\begin{array}{l}\text { Family } \\
\text { practice }\end{array}$ & Smoking & Single & - \\
\hline $\begin{array}{l}\text { Hemminki }^{51} \\
\text { (Finland } \text { 1990) }\end{array}$ & 1987 & Obstetrics & $\begin{array}{l}\text { None } \\
\text { (pregnancy) }\end{array}$ & Double & - \\
\hline $\begin{array}{l}\text { Cogan } 52 \\
(U S, 1988)\end{array}$ & - & Obstetrics & Prematurity & Double & - \\
\hline $\begin{array}{l}\text { Labrie }{ }^{53} \\
\text { (Canada, 1999) }\end{array}$ & 1988 & $\begin{array}{l}\text { Oncology } \\
\text { screening }\end{array}$ & Prostate cancer & Single & Cont \\
\hline $\begin{array}{l}\text { Garrdlund } \\
\text { (Sweden, 1996) }\end{array}$ & 1988 & $\begin{array}{l}\text { Internal } \\
\text { medicine }\end{array}$ & $\begin{array}{l}\text { Pulmonary } \\
\text { embolism }\end{array}$ & Single & Rand \\
\hline
\end{tabular}

* studies with two index groups and one reference group.

$\dagger$ Cont to avoid contamination; Rand: to avoid problems with tandomization; Other: other reason.

* no information given in the reviewed paper.

$\$$ R: data collected routinely or by means of a register; P: Data (also) collected by personal contact with participants. 


\begin{tabular}{llll}
\hline $\begin{array}{l}\text { Approved by } \\
\text { ethics } \\
\text { committee }\end{array}$ & $\begin{array}{l}\text { Intervention } \\
\text { Yes }\end{array}$ & $\begin{array}{l}\text { Radical irradiarion \& salvage } \\
\text { cystectomy } \\
\text { The 'Know your midwife' } \\
\text { schene } \\
\text { Chemotherapy }\end{array}$ & $\begin{array}{l}\text { Preoperative irradiation \& } \\
\text { cystectomy } \\
\text { Standard hospital care }\end{array}$ \\
registration
\end{tabular}


Table 2. Continued

\begin{tabular}{|c|c|c|c|c|c|}
\hline $\begin{array}{l}\text { 1st author ref } \\
\text { (country, year) }\end{array}$ & $\begin{array}{l}\text { Year } \\
\text { begun }\end{array}$ & $\begin{array}{l}\text { Area or } \\
\text { medicine }\end{array}$ & Disotder & Design: & $\begin{array}{l}\text { Main reason } \\
\text { use design }+\end{array}$ \\
\hline $\begin{array}{l}\text { de Lorgenil } 55,56 \\
\text { (1 rance, } 1999)\end{array}$ & 1988 & Cardiology & $\begin{array}{l}\text { Myocardial } \\
\text { infarction }\end{array}$ & Single & Cont \\
\hline $\begin{array}{l}\text { Holmberg } \\
\text { (Sweder, 2002) }\end{array}$ & 1989 & Oncology & Prostate cancer & $\begin{array}{l}\text { Single- } \\
\text { double }\end{array}$ & - \\
\hline $\begin{array}{l}\text { Brus }{ }^{58} \\
\text { (Netherlands, 1998) }\end{array}$ & $\dot{2}$ & Rheumatology & $\begin{array}{l}\text { Rheumatoid } \\
\text { arthritis }\end{array}$ & Double & Cont \\
\hline $\begin{array}{l}\text { Goy } 5260 \\
\text { (Switzerlland, 1999) }\end{array}$ & 1989 & Catdiology & Artery stenosis & Single & - \\
\hline $\begin{array}{l}\text { Mac Vicat } 61 \\
(\mathrm{UK}, 1993)\end{array}$ & 1989 & Obstetrics & $\begin{array}{l}\text { None } \\
\text { (jognancy) }\end{array}$ & Single & Cont \\
\hline $\begin{array}{l}\text { Scholes }{ }^{62} \\
(U S, 1996)\end{array}$ & 1990 & $\begin{array}{l}\text { Primary cate, } \\
\text { screening }\end{array}$ & $\begin{array}{l}\text { Chlamydial } \\
\text { infection }\end{array}$ & Single & Rand \\
\hline $\begin{array}{l}\text { Collins } 6.3 \\
\text { (UK, 1991) }\end{array}$ & - & Psychiatry & $\begin{array}{l}\text { Chronic } \\
\text { schizophrenia }\end{array}$ & Single & Rand \\
\hline $\begin{array}{l}\text { Cline } \\
\text { (Sweden, 1998) }\end{array}$ & 1991 & Cardiology & Heart failure & Double & Cont \\
\hline $\begin{array}{l}\text { Cherkin } \\
\text { (US, 1996) }\end{array}$ & 1992 & Primary care & Low back pain & Double & Cont \\
\hline $\begin{array}{l}\text { Dennis } \\
\text { (UK, 1997) }\end{array}$ & 1992 & Public health & Stroke & Single & - \\
\hline $\begin{array}{l}\text { Auvinen }{ }^{67} \\
\text { (Fimland, 2001) }\end{array}$ & 1993 & Urology & Prostate Cancer & Double & - \\
\hline $\begin{array}{l}\text { wan der Sande } 68 \\
\text { (Netherlands, } 1997)\end{array}$ & 1993 & Psychiatry & $\begin{array}{l}\text { Attempted } \\
\text { suicitde }\end{array}$ & Double & Rand \\
\hline $\begin{array}{l}\text { Torgerson } \\
\text { (UK, 1997) }\end{array}$ & 1993 & $\begin{array}{l}\text { Internal medy- } \\
\text { cine screening }\end{array}$ & Osteoporosis & Single & Cont \\
\hline
\end{tabular}

* studies with two index groups and one reference group.

† Cont: to avoid contamination; Rand to avoid problems with tandomization; Other: other reason.

* no information given in the reviewed paper.

9 R: data collected routinely or by means of a register; $\mathbb{P}$ : Data (also) collected by personal contact with patticipants. 


\begin{tabular}{|c|c|c|c|}
\hline $\begin{array}{l}\text { Approved by } \\
\text { ethics } \\
\text { committee }\end{array}$ & Intervention & Reference & $\begin{array}{l}\text { Baseline / } \\
\text { effect } \\
\text { registration }\end{array}$ \\
\hline Yes & Mediterranean diet & Prudent diet: & $\mathrm{R} / \mathrm{R}$ \\
\hline Yes & Radical prostatectomy & Warchful waiting & $\mathrm{R} / \mathrm{R}$ \\
\hline Yes & Education programme & $\begin{array}{l}\text { Brochure about } \\
\text { theumatoid arthritis }\end{array}$ & $\mathrm{P} / \mathrm{P}$ \\
\hline Yes & $\begin{array}{l}\text { Percutaneous trans-luminal } \\
\text { coronary angioplasty }\end{array}$ & $\begin{array}{l}\text { Cotonary artery bypass } \\
\text { grafting }\end{array}$ & $\mathrm{R} / \mathrm{R}$ \\
\hline Yes & Home from Home & Routine care & $\mathrm{R} / \mathrm{R}$ \\
\hline Yes & Chlamydia trachomatis test & Usual care & $\mathrm{P} / \mathrm{P}$ \\
\hline Yes & Lithium carbonate & Standard care & $\mathrm{P} / \mathrm{P}$ \\
\hline Y'es & Education & Routine clinical practice & $\mathrm{R} / \mathrm{P}$ \\
\hline Yes & Educational interventions & Usual care & $\mathrm{P} / \mathrm{P}$ \\
\hline Yes & Stroke family care worker & Standard care & $\mathrm{R} / \mathrm{P}$ \\
\hline Yes & $\begin{array}{l}\text { Active role patient in } \\
\text { treatment choice }\end{array}$ & Usual Care & $\mathrm{R} / \mathrm{P}$ \\
\hline - & Psychosocial treatment & Care as usual & $\mathrm{P} / \mathrm{R}$ \\
\hline- & Osteoporosis screening & No screening & $\mathrm{P} / \mathrm{P}$ \\
\hline
\end{tabular}


Talste 2. Continued.

\begin{tabular}{|c|c|c|c|c|c|}
\hline $\begin{array}{l}1 \text { at anhor the } \\
\text { (country, year) }\end{array}$ & $\begin{array}{l}\text { Year } \\
\text { begun }\end{array}$ & $\begin{array}{l}\text { Area of } \\
\text { medicine }\end{array}$ & Disorder & Design & $\begin{array}{l}\text { Main reason } \\
\text { we design }\end{array}$ \\
\hline $\begin{array}{l}\text { Uambett } \\
(\mathrm{UK}, 1998)\end{array}$ & 1994 & Rheumatology & $\begin{array}{l}\text { Rheumatoid } \\
\text { arthritus }\end{array}$ & Double & - \\
\hline $\begin{array}{l}\text { Vierhout } \\
\text { Wetherlands, } 1995 \text { ) }\end{array}$ & - & Otthopedics & $\begin{array}{l}\text { Locomotot- } \\
\text { system disorders }\end{array}$ & Double & Cont \\
\hline $\begin{array}{l}\text { Dey } \\
\text { (UK, 2002) }\end{array}$ & 1995 & Oncology & Breast lump & Double & Rand \\
\hline $\begin{array}{l}\text { Zhu }{ }^{73} \\
\text { (US, 2002) }\end{array}$ & 1995 & Public health & Smoking & Double & Cont \\
\hline $\begin{array}{l}\text { Hux } \\
\text { (Canada, 1999) }\end{array}$ & 1995 & Primary care & $\begin{array}{l}\text { None (prescrip- } \\
\text { tion antibiotics) }\end{array}$ & Double & Cont \\
\hline $\begin{array}{l}\text { Willsdon * } 75 \\
\text { (UK, 2002) }\end{array}$ & 1996 & Primary care & None & Single & Other \\
\hline $\begin{array}{l}\text { Rodgerss } 76 \\
(\mathrm{UK}, 1999)\end{array}$ & 1997 & Public health & Stroke & Double & - \\
\hline $\begin{array}{l}\text { Streiner } \\
\text { (UK, 2001) }\end{array}$ & 1997 & Geriattics & $\begin{array}{l}\text { None (needs } \\
\text { elderly people) }\end{array}$ & Single & Rand \\
\hline $\begin{array}{l}\text { Homer } \\
\text { (Australia, 2001) }\end{array}$ & 1997 & Obstetrics & $\begin{array}{l}\text { None } \\
\text { (pregnancy) }\end{array}$ & Double & Cont \\
\hline $\begin{array}{l}\text { Pinto } 79 \\
\text { (Spain, 2002) }\end{array}$ & 1999 & Radiology & Uterine fibroids & Single & - \\
\hline
\end{tabular}

* studies witl two index groups and one reference group.

+ Cont: to avoid contamination; Rand: to avoid problems with randomization; Other: other reason.

* no infomation given in the reviewed paper.

1. R: data collected routinely or by means of a register; $P$ : Data (also) collected by personal contact with participants. 


\begin{tabular}{|c|c|c|c|}
\hline $\begin{array}{l}\text { Approved by } \\
\text { ethics } \\
\text { committee }\end{array}$ & Intervention & Reference & $\begin{array}{l}\text { Baseltine/ } \\
\text { effect } \\
\text { registration }\end{array}$ \\
\hline Yes & Day care & Inpatient care & $\mathrm{P} / \mathrm{p}$ \\
\hline Yes & Joint consultation sessions & Usual care & $\mathrm{p} / \mathrm{p}$ \\
\hline Yes & One stop clinic & Dedicated breast clinic & $\mathrm{P} / \mathrm{P}$ \\
\hline- & Counseling sessions & Self-thelp materials & $\mathrm{P} / \mathrm{P}$ \\
\hline Yes & Confidentially profiles & No intervention & $\mathrm{R} / \mathrm{P}$ \\
\hline Yes & $\begin{array}{l}\text { Brief negotiation } \\
\text { Direct advice }\end{array}$ & No intervention & $P / P$ \\
\hline Yes & Stroke education program & $\begin{array}{l}\text { Conventional stroke unit } \\
\text { care }\end{array}$ & $\mathrm{p} / \mathrm{p}$ \\
\hline Yes & Nurse-led unit & Conventional care & $\mathrm{R} / \mathrm{P}$ \\
\hline Yes & Community-based care & $\begin{array}{l}\text { Standard hospital-based } \\
\text { care }\end{array}$ & $\mathrm{R} / \mathrm{R}$ \\
\hline Yes & Uterine uttery embolization & Abdominal hysterectomy & $\mathbb{R} / \mathbb{R}$ \\
\hline
\end{tabular}

\section{Rates of non-compliance and loss to follow-up}

Table 3 shows the rates of non-compliance and loss to follow-up for each study design. Table 4 summarizes table 3 by presenting the median rates and interquartile ranges (IQR). For the incomplete-double-consent design, the median rate of noncompliance and loss to follow up is abour $15 \%$ in both study groups. In the reference groups of the trials using an incomplete-double-consent design, the rates of non-compliance and loss to follow-up were statistically significantly higher than in the trials that used the other two designs. To explore these differences, Figure 1 plots year of study commencement against the field of study (cancer, other diseases and behavior) for the three types of design. All four studies with a completedouble-consent design were cancer trials that were started before 1985. Figure 1 shows that, after 1990 the randomized consent design was no longer used in 
cancer trials, whereas the use of the incomplete-double-consent design is restricted to the behawional field.

As dissatisfaction with the allocated treatment leading to non-compliance and loss to follow-up is an important reason to use a randomized consent design, we plotted the difference of non-compliance and loss to follow-up between the index and reference group against the subject of study for the three types of design (Figures $2 \mathrm{a}$ and $2 \mathrm{~b}$ ). In $65 \%$ of the studies the non-compliance in the index group is larger than in the reference group. For the loss to follow-up this percentage is $32 \%$. Furthermore there is no correlation between these differences and the year of study ( $r=0.01$, and 0.03 , respectively). 
Table 3. Rate of non-compliance and loss to followm up in randomized single-consent and double-consent designs.

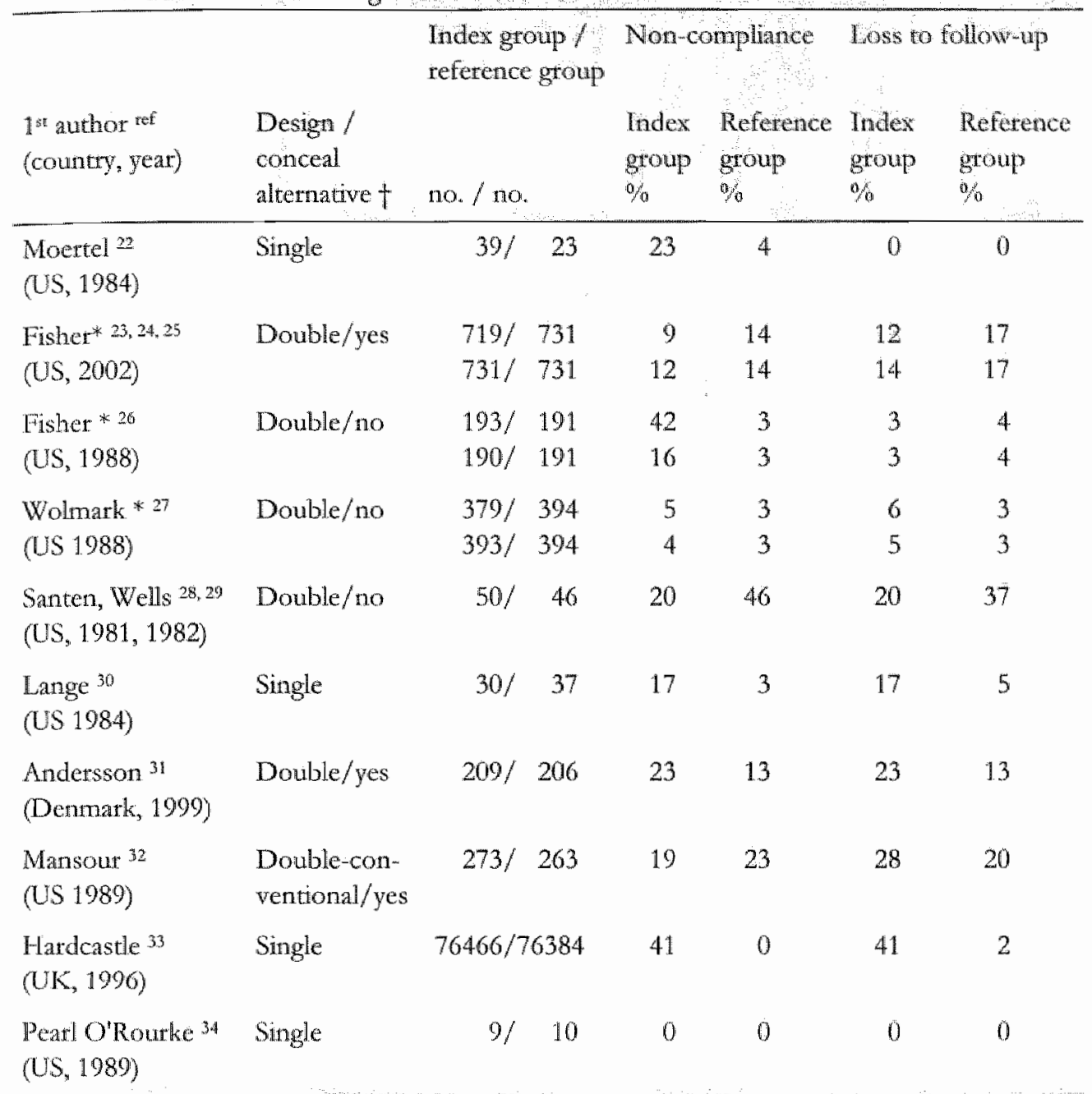

* studies with two experimental groups and one reference group.

t yes: participants are not informed about alternative treatment. no: participants are fully informed about alternative treatment.

* data cannot be calculated or traced. 
Table 3. Continued.

\begin{tabular}{|c|c|c|c|c|c|c|}
\hline \multirow[b]{2}{*}{$\begin{array}{l}1^{\text {st }} \text { author ref } \\
\text { (country, year) }\end{array}$} & \multirow[b]{2}{*}{$\begin{array}{l}\text { Design / } \\
\text { conceal } \\
\text { alternative } \dagger\end{array}$} & $\begin{array}{l}\text { Index group / } \\
\text { reference group }\end{array}$ & \multicolumn{2}{|c|}{ Non-compliance } & \multicolumn{2}{|c|}{ Loss to follow-up } \\
\hline & & no. / no. & $\begin{array}{l}\text { Index } \\
\text { group } \\
\%\end{array}$ & $\begin{array}{l}\text { Reference } \\
\text { group } \\
\%\end{array}$ & $\begin{array}{l}\text { Index } \\
\text { group } \\
\%\end{array}$ & $\begin{array}{l}\text { Refetence } \\
\text { group } \\
\%\end{array}$ \\
\hline $\begin{array}{l}\text { Bartlett } 35 \\
\text { (US, 1985) }\end{array}$ & Single & $11 / 1$ & 0 & 0 & 0 & 0 \\
\hline $\begin{array}{l}\text { Hejrisis } 36,37,38 \\
\text { (Denmark, 1999) }\end{array}$ & Double/yes & $1610 / 1639$ & 5 & 7 & 4 & 6 \\
\hline $\begin{array}{l}\text { Balch }{ }^{39} \\
\text { (US, 2001) }\end{array}$ & Double/yes & $238 / 230$ & $-\ddagger$ & - & - & - \\
\hline $\begin{array}{l}\text { Blichert-Toft } 40,41 \\
\text { (Denmark, 1992) }\end{array}$ & $\begin{array}{l}\text { Single- } \\
\text { doublie/yes }\end{array}$ & $450 / 455$ & 17 & 14 & 4 & 6 \\
\hline $\begin{array}{l}\text { Sell }{ }^{42} \\
\text { (Denmatk, 1991) }\end{array}$ & Single & $95 / \quad 88$ & 7 & 13 & 0 & 0 \\
\hline $\begin{array}{l}\text { Fint }{ }^{43} \\
(\mathrm{UK}, 1989)\end{array}$ & Double/yes & $503 / 498$ & 9 & 0 & 3 & 4 \\
\hline $\begin{array}{l}\text { Ganz } \\
(\text { US, 1988) }\end{array}$ & Double/no & $31 / 32$ & 19 & 19 & 29 & 19 \\
\hline $\begin{array}{l}\text { Hemmintaki }{ }^{45} \\
\text { (Finland, 1989) }\end{array}$ & Double/yes & $1358 / 1336$ & 6 & 5 & - & 14 \\
\hline $\begin{array}{l}\text { Kronborg }{ }^{46} \\
\text { (Denmark, 1996) }\end{array}$ & Single & $30967 / 30966$ & 33 & 0 & 0 & 0 \\
\hline $\begin{array}{l}\text { Rierthmuiller }{ }^{47} \\
\text { (Germany, 1994) }\end{array}$ & Single & $99 / \quad 90$ & 14 & 0 & 9 & 16 \\
\hline
\end{tabular}


Table 3. Continued.

Index group Non-compliance Loss to tollow up reference group

\begin{tabular}{|c|c|c|c|c|c|c|c|}
\hline $\begin{array}{l}1^{\text {st }} \text { author } \\
\text { (country, year) }\end{array}$ & $\begin{array}{l}\text { Design / } \\
\text { conceal } \\
\text { alternative } \dagger\end{array}$ & no. / no. & & $\begin{array}{l}\text { Index } \\
\text { group } \\
\%\end{array}$ & $\begin{array}{l}\text { Reference } \\
\text { group } \\
\%\end{array}$ & $\begin{array}{l}\text { Tndex } \\
\text { group } \\
\%\end{array}$ & $\begin{array}{l}\text { Refrence } \\
\text { group } \\
0\end{array}$ \\
\hline $\begin{array}{l}\text { Korvick } \\
\text { (UK, } 1992)\end{array}$ & Single & $58 \%$ & 63 & 10 & 0 & 0 & 0 \\
\hline Bryce ${ }^{49}$ & Single & $983 /$ & 987 & 9 & 0 & 0 & 0 \\
\hline
\end{tabular}

(A.ustralia, 1991)

Lambe ${ }^{50}$

Single

$90 / \quad 90 \quad 27 \quad 0$

$33 \quad 33$

(US, 1986)

Hemminki ${ }^{51}$

Double/yes $\quad>122 />118$

$-1$

7

(Finland, 1990)

Cogan $^{52}$

Double/yes

$20 / 14$

5

0

35

21

(US, 1988)

Labrie ${ }^{53}$

Single

$30956 / 15237 \quad 77$

6

0

0

(Canada, 1999)

Gardlund $^{54}$

Single

$5776 / 5917 \quad 12$

12

(Sweden, 1996)

de Lorgeril ${ }^{35,56}$

Single

$302 / 303$

27

0

22

20

(France, 1999)

Holmberg ${ }^{57}$

Single-

$349 / 349$

16

6

0

0

(Sweden, 2002)

double/yes

Butus 58

Double/yes

$32 / 33$

9

6

22

9

(Netherlands, 1998)

* studies with two experimental groups and one refenence group.

+ yes: participants are not informed about altemative treatment. no: participants are fully informed about alternative treatment.

\$ data cannot be calculated or traced. 
Index group / Non-compliance Loss to follow up reference group

\begin{tabular}{|c|c|c|c|c|c|c|}
\hline $\begin{array}{l}1^{\text {s }} \text { athor } \\
\text { (country, geas) }\end{array}$ & $\begin{array}{l}\text { Design / } \\
\text { conceal } \\
\text { alternative } \dagger\end{array}$ & no. $/$ no. & $\begin{array}{l}\text { Index } \\
\text { group } \\
\%\end{array}$ & $\begin{array}{l}\text { Reference } \\
\text { group } \\
\%\end{array}$ & $\begin{array}{l}\text { Index } \\
\text { group } \\
\%\end{array}$ & $\begin{array}{l}\text { Reference } \\
\text { group } \\
\%\end{array}$ \\
\hline $\operatorname{Coy} 51,60$ & Single & $68 / 66$ & 0 & 8 & 0 & 0 \\
\hline
\end{tabular}

(Switzerland, 1999)

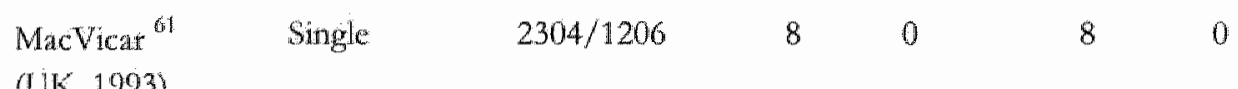

(UK, 1993)

Scholes ${ }^{62}$

Single

$1009 / 1598$

36

0

$+$

(US, 1996)

Collins ${ }^{63}$

Single

21/ 23

52

4

52

4

(UK, 1991)

Cline $^{64}$

Double/yes

96/ 110

17

0

42

28

(Sweden, 1998)

Cherkin ${ }^{65}$

Double/yes

$262 / 129$

$26 \quad 25$

28

28

(US, 1996)

Dennis ${ }^{66}$

Single

$210 / 207$

$0 \quad 0$

11

11

(UK, 1997)

Auvenin ${ }^{67}$

Double/yes

$105 / 105$

25

2

5

(Finland, 2001)

van der Sande ${ }^{68}$

Double/yes

$140 / 134$

18

48

0

(Netherlands, 1997)

Torgerson ${ }^{69}$
(UK 1997$)$

Single

$799 / 800$

29

0

23

24

I. ambert ${ }^{70}$

Double/yes

$59 / 59$

19

3

14

14

(UK, 1998)

\footnotetext{
* studies with two experimental groups and one reference group.

$\dagger$ yes: participants are not informed about alternative treatment. no: participants are fully informed about alternative treatment.

* data eannot be calculated or traced.
} 
Table 3. Continued.

Index group / Non-compliance Los to follow up refenence group

$1^{\text {st }}$ author reit Design/

Index Reference Tadex Reference

(country, year)

conceal group group group group alternative +

no. $/ \mathrm{no}$

$6 \%$

Verhout $^{71}$
Netherlands, 1995)

Dey $^{72}$

Double/yes

$351 / 344$

$24 \quad 39$

41

56

(UK, 2002)

Zha ${ }^{73}$

Double/yes $\quad 1973 / 1309$

$28 \quad 32$

29

30

(US, 2002)

$\operatorname{Hux}^{74}$

Double/yes $\quad 400 / 400$

60

71

66

71

(Canada, 1999)

Hillsdon * ${ }^{75}$

Single

$551 / 563$

45

0

68

43

(UK, 2002)

$544 / 563$

48

67

43

Rodgers

Double/yes

$121 / 83$

58

0

26

23

(UK, 1999)

Steiner ${ }^{77}$

Single

$119 / \quad 121$

10

0

28

44

(UK, 2001)

Homer $^{78}$

Double/yes $640 / 643$

25

21

25

21

(Australla, 2001)

Pinto ${ }^{79}$

Single

$38 / 19$

316

0

0

(Spain, 2002)

* studies with two experimental groups and one reference group.

+ yes: patticipants are not informed about altcrnative treatment. no: participarts are fully informed about alternative treatment.

* data cannot be calculated or traced. 
Table 4. The median rates and Inter Quartile Range (TQR) of non-compliance and loss to follow-up in the index and the reference group in the incomplete-doubleconsent design, the complete-double-consent design and the single-consent designa.

\begin{tabular}{|c|c|c|c|c|}
\hline & $\begin{array}{l}\text { Median tate } \\
\text { non-compliat }\end{array}$ & $\begin{array}{l}\text { IQR) of } \\
\text { ce }(\%)\end{array}$ & $\begin{array}{l}\text { Median rate ( } \\
\text { loss to follow }\end{array}$ & $\begin{array}{l}\text { QR) of } \\
\text { up }(\%)\end{array}$ \\
\hline & Index group & Reference group & Index group & Reference group \\
\hline $\begin{array}{l}\text { Incomplete-dovible- } \\
\text { consent design }(n=23)\end{array}$ & $17 \quad(9-24)$ & $14 \quad(4-23)$ & $15(4-28)$ & $17(5-23)$ \\
\hline $\begin{array}{l}\text { Complete-double- } \\
\text { consent design }(n=4) *\end{array}$ & $18(5-32)$ & $3(3-25)$ & $5(3-22)$ & $4 \quad(3-23)$ \\
\hline $\begin{array}{l}\text { Single-consent design } \\
(n=23)\end{array}$ & $15 \quad(7-39)$ & $0 \quad(0-4)$ & $9 \quad(0-28)$ & $0(0.24)$ \\
\hline $\begin{array}{l}\text { Test for difference } \\
\text { (p-yalue) } 1\end{array}$ & 0.99 & $<0.0001$ & 0.32 & 0.04 \\
\hline
\end{tabular}

These frour studies represent two studies with one index group and two studies with two index groups. Data analysis is based on six index groups.

I Kruskal-Wallis test

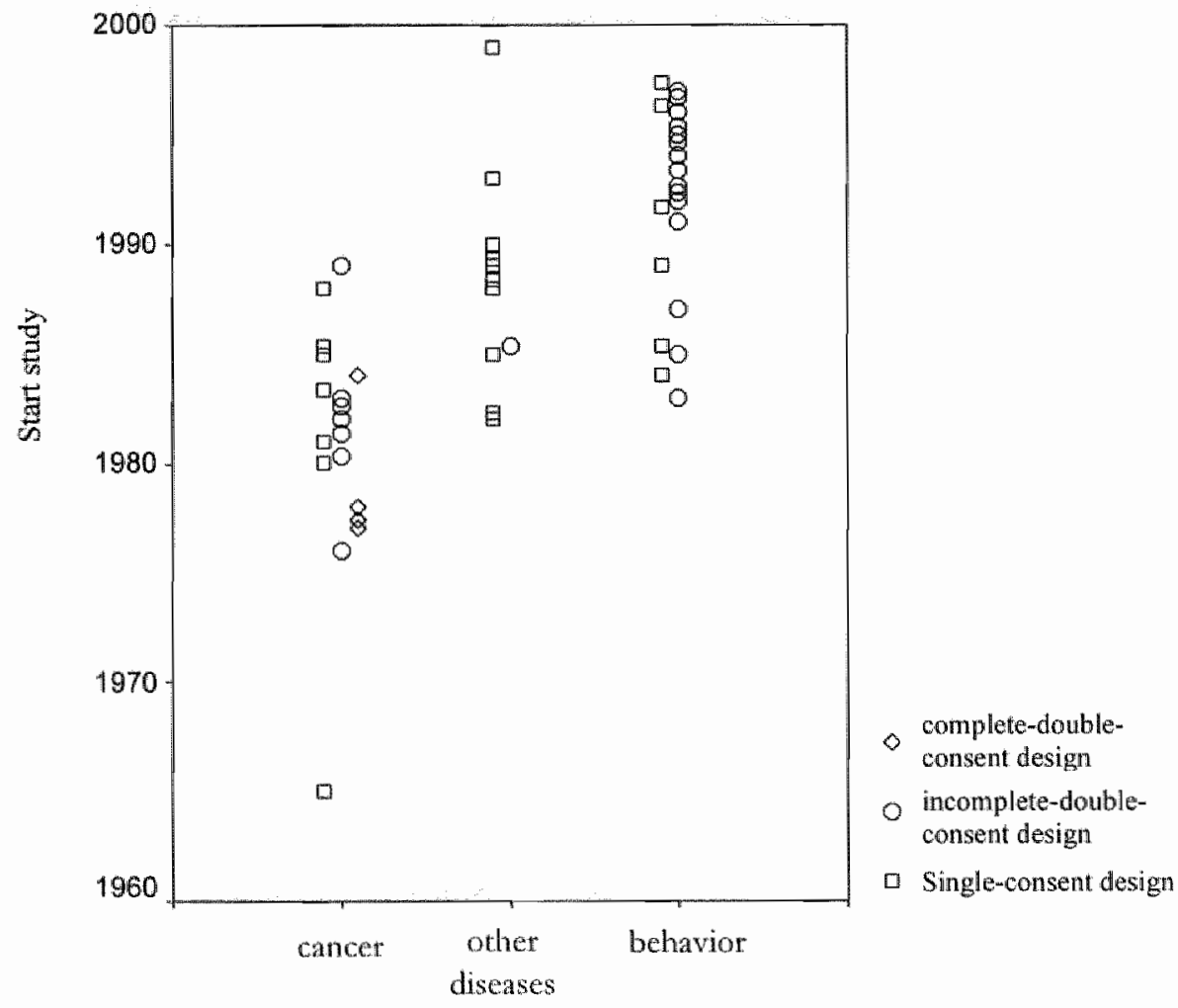

Subject of study

Figure 1. Year of study against the subject of srudy for the complete double-design, the incomplete-double-design, and the single-consent design $(\mathrm{N}=50)$. 


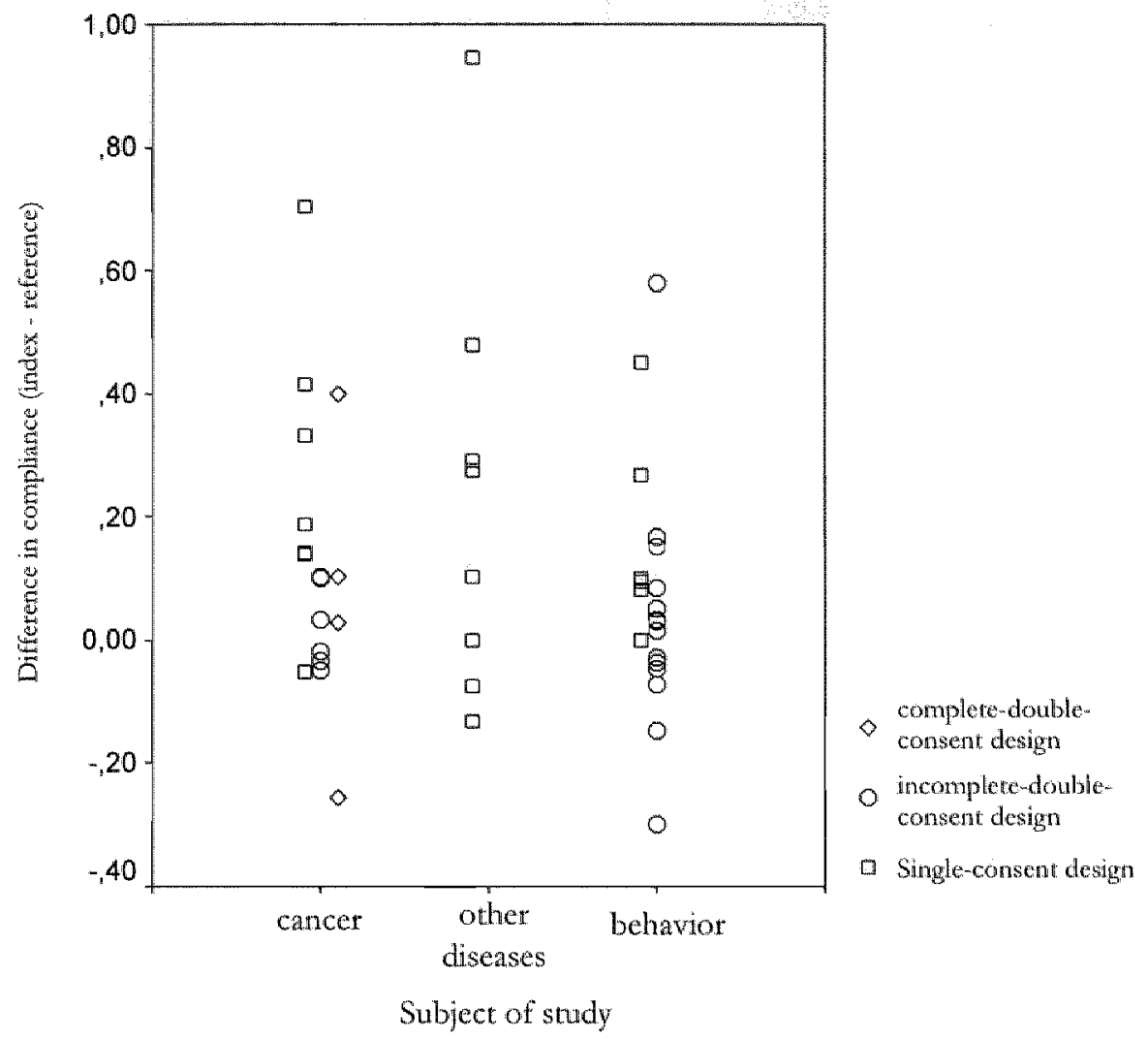

Figure 2a. Difference in compliance (rate of non-compliance index gtoup minus tate of non-compliance reference group) against the subject of study for the complete-double-design, the incomplete-double-design, and the single-consent design $(\mathrm{N}=50)$. 


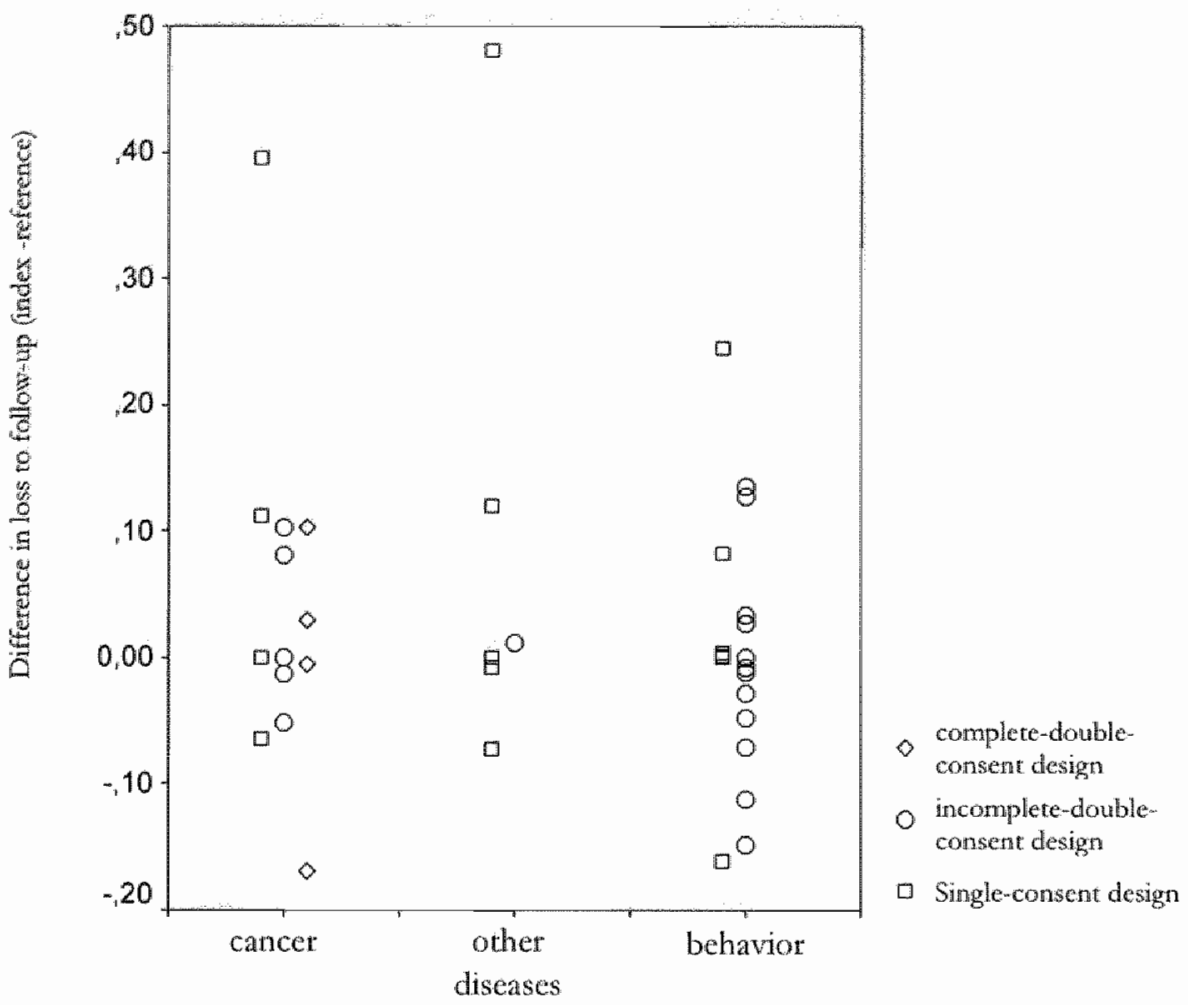

Subject of study

Figure 2b. Difference in loss to follow-up (rate of loss to follow-up index group minus rate of loss to follow-up reference group) against the subject of study for the complete-double-design, the incomplete-double-design, and the single-consent design $(\mathrm{N}=50)$.

\section{Discussion}

Our search strategy identified 50 studies that used a randomized single-consent or a double-consent design. Although we did our search systematically and painstakingly it cannot be excluded that we missed some trials.

In 1982 Zelen predicted that randomized consent designs would dominate the field of randomized clinical trials ${ }^{3}$ However, the absolute number of published trials using these designs appears to be extremely low, which is more in accordance with the expectation of some other authors. $5,80,81$ Until the mid-Nineteen-Eighties, the randomized consent design was mainly used in oncology trials in the U.S. and in Scandinavia. Probably due to changing of federal guidelines that restricted the use of the randomized consent design ${ }^{8,9}$ the double-consent became the choice of 
preference. This double-consent approach is still permitted under federal US regulations today. ${ }^{5}$

Both forms of the double-consent design can be disputed. The incomplete informed consent procedure might be considered as unethical. ${ }^{82}$ Our review shows that research ethics committees approved at least 23 trials using a double-consent design. The complete-double-consent design, as used in four US cancer trials in the late-Seventies and early-Eighties, informs the participants in both treatment arms about all the study features. Compared to the traditional trial design, only the timing of information relative to the act of tandomization differs. Therefore, one might expect the withdrawal rates to be similar, although possibly unbalanced between the study groups. Therefore, the conventional randomized design is methodologically preferable.

In 11 studies it was indicated that expected problems with the randomization procedure was the main reason for using the randomized consent design. However, the application of the randomized consent design should not have found support from research ethics committees if the main reason is to reduce the work of recruiting patients and decrease the burden for investigators as was reported in references 5,20 , and $82-84$.

The differences in non-compliance rates berween the complete-double-consent design and the other two designs may be attributed to the fact that the completedouble-consent designs were cancer trials with unattractive and burdening interventions $\mathrm{s}^{26,27-29,44}$ and non-invasive reference interventions. $26,27,44$ The low loss to follow up rates in the complete-double-consent designs may be explained by data collection for effect measurements by means of a register, without personal contact with the patients. ${ }^{26-29}$

We have previously argued that if the experimental intervention is attractive and contamination is likely, a randomized consent design would reduce noncompliance and loss to follow-up in the reference group. $19,20,85,86$ However, as Figures $2 a$ and $2 b$ show, in the majority of the studies the rates of non-compliance and loss to follow-up were greater in the index groups than in the reference groups. This might indicate that these studies did not fulfill our atractiveness and contamination requirements.

In his teview of cancer trials, Altman et al. concluded that there are serious statistical arguments against the use of randomized consent designs, which should discourage their use ${ }^{80}$ Reticence about using a randomized consent designs was expressed also by Ellenberg." Given the experiences in some cancer trials using a randomized consent design, Ellenberg argued that, because of scientific concens (contamination), and ethical concerns (incomplete information), the application of these designs should be considered a last-resort measure. We agree with Altman et al. and Ellenberg that these designs should not be used without compelling reason. However, situations requiring a randomized consent design do exist, for instance, 
in case of a heroin-provision experiment ${ }^{9}$. Summing up, the number of trials in which a randomized consent design was used is very small; the complete-doubleconsent design has not been used since 1985; over years the subject of the studies changed from medical to behavional interventions; and in $22 \%$ of the studies problems expected with the randomization procedure was reported as the main reason for using a randomized consent clesign. We think that for the majority of the studies reviewed here, the use of a randomized consent design was unjustified.

In our opinion; the use of randomized consent designs should be restricted to studies in which contamination is otherwise tnavoldable. More importantly, the significance of the study question should be in balance with the infringement of the participant's rights ${ }^{15}$. Therefore, a tesearch ethics committee should decide whether this balance is right in any particular study and the application of the randomized consent design justified. 


\section{References}

1. Zelen M. Statistical options in clinical trials. Semin Oncol 1977, 4:441-46.

2. Zelen M. A new design for tandomized clinical trials. N Engl J Med 1979; 300: 1242-5.

3. Zelen M. Strategy and alternate randomized designs in cancer clinical trials. Cancer Treat Rep 1982; 66: 1095-1100.

4. Ellenberg SS. Randomization designs in comparative clinical trials. New Engl J Med $1984 ; 310: 1404-08$.

5. Ellenberg SS. Informed consent protection or obstacle? Some emerging issues. Control Clin Trials $1997 ; 18: 628-36$.

6. Beauchamp TL, Cook RR, Fayerweather WE, Raabe GK, Thar WE, Cowles SR, Spivey GH. Ethical guidelines for epidemiologists. J Clin Epidemiol 1991; 44: 151S.69S.

7. Gezondheidstaad. Informed consent en prexandomisatie. Den Hag: Gezondheidsraad, 1999.

8. Medical Research Council Cancer Therapy Committee. Investigations on human subjects: ethical considerations in the study of cancer therapy. CTG $86 / 24$, February 1987.

9. Final rule-final regulations amending basic HHS policy for the protection of human research subjects (45 CFR Part 46). Federal Register. January 26, 1981; 46: 8366m91.

10. Zelen M. Alternatives to classic randomized trials. Surg Cin N Am 1981; 61: 1425-32

11. Zelen M. Innovations in the design of clinical trials in breast cancer. Breast Cancer Res Treat $1983 ; 3: 137-42$.

12. Zelen M. Randomized consent designs for clinical trials: an upclate. Stat Med 1990; 9: $645-46$.

13. Anbar $D$. The relative efficiency of Zelen's pretandomization design for clinical trials. Biometrics 1983; 39: 711-18.

14. Matts J, McHugh R. Randomization and efficiency in Zelen's single-consent design. Biometrics 1987; 43: 885-94.

15. Ashcroft RE, Chadwick DW, Clatk SRI, Edwards RHT, Fith L, Hutton J... Implications of socio-cultural contexts for ethics of clinical trials. Health Technol Assessment $1997 ; 1: 1-67$.

16. Edwards SJL, Lilfotd RJ, Brannholtz DA, Jackson JC, Hewison J, Thornton J. Eicthical issues in the design and conduct of randomised controlled trials. Health Technol Assessment $1998 ; 2$ (15).

17. Homer CS. Using the Zelen design in randomized controlled trials: debates and controversies.] Adv Nurs 2002; 38: 200-7.

4. Palmer CR. Ethics, data-dependent designs, and the strategy of clinical trials: time to start learning aswwe-go? Stat Methods Med Res 2002; 11: $381-402$.

19. Schellings $R$, Kessels $A G$, ter Riet $G$, Sturmans $F$. "The Zden design may be the best choice for a heroin-provision experiment. J Clin Epidemiol 1999; 52: 503-07.

20. Knottnerus $\mathrm{J} A$. Health care research in ambulatory care. In: Ethiek en recht in de gezondheidszorg (Dutch language). Deventer: Kluwer; 1997. p. IV 151-94.

21. Butren $\mathrm{S}$ van, Boshuizen $\mathrm{HC}$, Knook DL. Multiple imputation of missing blood pressure covariates in survival analysis. Stat Med 1999; 18; 681-94.

22. Moertel CG, Childs DS, O'Fallon JR, Holbrook MA, Schutt AJ, Reitemeter RJ. Combined 5 -fluorouracil and radiation therapy as a surgical adjurant for poor prognosis gastric carcinona. J Clin Oncol 1984; $2: 1249-54$. 
23. Tushet $B$, Bauer $M$, Margolese $\mathbb{R}_{\text {, Poisson }} \mathrm{R}_{3}$ Pilch $\mathrm{Y}$, Redmond $C_{\text {, }}$ et al. Five-year results of a fandomized clinical trat comparing total mastectomy and segmental mastectomy with or whout radiaton in the treatment of breast cancer. $N$ Engl Med $1985 ; 312: 665-73$.

24. Fisher $B$, Redmond $C$, Poisson $R$, Margolese $R$, Wolmark $N$, Wickerham $\mathbb{L}$, et al. Eightyear tesults of randomized clinical trial comparing total mastectomy and himpectomy with or without irradiation in the teament of breast cancer. N Engl ] Med 1989; 320 : $822-8$

25. Fisher B, Anderson S, Bryant J, Margolese RG, Deutsch $M$, Fisher ER, et al. Twentyyear follow-up of a randomized trial comparing total mastectomy, lumpectomy, and umpectomy plus irradiation for the treatment of invasive breast cancer. $\mathrm{N}$ Engl J Med 2002; 347: 1233441.

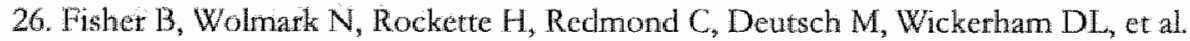
Postoperative adjuvant chemotherapy or radiation therapy for rectal cancer: results from NSABP ptotocol R-01. J Natl Cancer Inst 1988; 80: 21-9.

27. Wolmark N, Fisher B, Rockette H, Redmond C, Wickerham DI, Fisher ER, et all. Postoperative adjuvant chemotherapy or BCG for colon cancer: results from NSABP protocol C-01. I Natl Cancer Inst 1988; 80:30-6.

28. Santen RJ, Worgul TJ, Samojlik E, Interrante A, Boucher AE, Lipton A, et al. A randomized trial comparing surgical adrenalectomy with aminoglutethimide plus hydrocortisone in women with adwanced breast cancer. N Engl] Med 1981; 305: 54551.

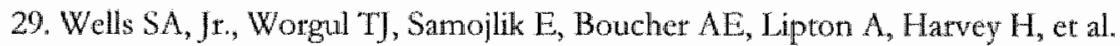
Comparison of surgical adrenalectomy to medical adrenalectomy in patients with metastatic carcinoma of the breast. Cancer Res 1982; 42 (Suppl): $3454 \mathrm{~s}-57 \mathrm{~s}$.

30. Lange B, Halpern S, Galle G, Kramer S. Trimethoprim-sulfamethoxazole and nystatin prophylaxis in children with acute lymphoblastic leukemia. Eur Paedriatr Haematol Oncol 1984; 1: 231-38.

31. Andersson M, Madsen EL, Overgaard M, Rose C, Dombernowsky P, Mouridsen HT. Doxorubicin versus methotrexate both combined with cyclophosphamide, 5fuorouracil and tamoxifen in postmenopausal patients with advanced breast cancer: a randomised study with more than 10 years follow-up from the Danish Breast Cancer Cooperative Group. Danish Breast Cancer Cooperative Group (DBCG). Eur J Cancet $1999 ; 35: 39-46$.

32. Mansour $\mathrm{EG}$, Gray R, Shatilla AF, Osborne CK, Tormey DC, Gilchrist KW, et al. Efficacy of adjuwant chemotherapy in high-risk node-negative breast cancer. An intergtoup study. N Engl J Med 1989; 320: 485-90.

33. Hardcastle JD, Chamberlain JO, Robinson MH, Moss SM, Amat SS, Balfour TW, et al. Randomised controlled trial of fatecal-occult-blood screening for colorectal cancer. Lancet $1996 ; 348: 1472 ;$.

34. O'Rourke PP, Crone RK, Vacanti JP, Ware JH, Lillehei CW, Parad RB, et al. Extracorporeal membrane oxygenation and conventional medical therapy in neonates with persistent pulmonary hypertension of the newborn: a prospective randomized study. Pedtatrics 1989; 84: 957-63.

35. Bartlett RH, Roloff DW, Cornell RG, Andrews AF, Dillon PW, Zwischenberger JB. Extracorpoteal circulation in neonatal respiratory failure: a prospective randomized study. Pediatrics $1985 ; 76: 479-87$. 
36. Hojris I, Overgaard $M$, Christensen JI, Overgard J. Morbidity and montulity of ischaemic heart disease in high-risk breast-cancer pattents after adjurant postmastectomy systemic reatment with or without radiotherapy: analysis of DBCG $82 \mathrm{~b}$ and $82 \mathrm{c}$ randomised trials. Radrotherapy Committee of the Danish Breast Cancet Cooperative Group. Lancet 1999; 354: 1425-30.

37. Overgaand M, Hansen PS, Overgated J, Rose C, Andersson M, Bach F, ar al. Postoperative tadiotherapy in high-risk premenopausal women with breast cancer who receive adjuvant chemotherapy. Danish Breast Cancer Cooperative Group $82 \mathrm{~b}$ Trial. N Engl II Med 1997; 337: 949 55 .

38. Overgaard M, Jensen MB, Owergaard J, Hansen PS, Rose C, Andersson M, et al. Postoperative radiotherapy in high-risth postmenopausal breast-cancet pationts given adjuxant tamoxifen: Danish Breast Cancer Cooperative Group DBCG $82 \mathrm{c}$ randomised trial. Lancet 1999; 353: 1641-8.

39. Balch CM, Soong S1, Smith T, Ross MI, Urist MM, Karakousis CP, et al. Long-tem resuls of a prospective surgical trial comparing $2 \mathrm{~cm} v \mathrm{~s} .4 \mathrm{~cm}$ excision margins for 740 patients with 14 mm melanomas. Ann Surg Oncol 2001; 8: 101-8.

40. Blichert-Toft $M$, Brinker $H$, Andersen JA et al. A randomized trial comparing breastpreserving therapy with mastectomy in mammary carcinoma. Acta Oncol 1988; 27 : 671.77 .

41. Blichert-Toft M, Rose C, Andersen $J A$, Overgaard M, Axelsson CK, Andersen KW, et al. Danish randomized trial comparing breast conservation therapy with mastectomy: six years of life-table analysis. Danish Breast Cancer Cooperative Group. I Natl Cancet Inst Monogr 1992 (11): 19-25.

42. Sell A, Jakobsen A, Nerstrom B, Sorensen BL, Steven K, Barlebo H. Treatment of advanced bladder cancer category $\mathrm{T} 2 \mathrm{~T} 3$ and $\mathrm{T} 4 \mathrm{a}$. A randomized multicenter study of preoperative irradiation and cystectomy versus radical iradiation and early salvage cystectomy for residual tumor. DAVECA protocol 8201. Danish Vesical Cancer Group. Scand J Urol Nephrol Suppl 1991; 138: 193-201.

43. Flint C, Poulengeris P, Grant A. The 'Know Yout Midwife' scheme--a randonised trial of continuity of cate by a team of midwives. Midwifery $1989 ; 5(1): 11-6$.

44. Ganz PA, Haskell CM, Figlin RA, La Soto N, Siau J. Estimating the quality of life in a clinical trial of patients with metastatic lung cancer using the Karnofsky performance status and the Functional Living Index-Cancer. Cancer 1988; 61: 849-56.

45. Hemminki $\mathbb{E}$, Uski $A$, Koponen P, Rimpela $U$. Iron supplementation during pregnancy. -experiences of a randomized trial relying on health service personnel. Control Clin Trials $1989 ; 10.290-8$.

46. Kronborg O, Fenger C, Olsen J, Jorgensen OD, Sondergaard O. Randomised study of screening for colorectal cancer with faccal-occult-blood test Lancet $1996 ; 348: 1467-71$.

47. Riethmuller G, Schneider-Gadicke $\mathbb{E}$, Schlimok G, Schmicgel W, Rab R, Hoffen K. et al. Randomised trial of monoclonal antibody for adjuvant therapy of resected Dukes' C colorectal carcinoma. German Cancer Aid 17-1A Study Group. Lancet 1994; 343: $1177-83$.

48. Korvick JA, Peacock JE, Jr, Muder RR, Wheeler RR, Yu VL. Addition of rifampin to combination antibiotic therapy for Pseudomonas acruginosa bacteremia: prospective trial using the Zelen protocol Antmicrob Agents Chemother 1992; 36: 620-5.

49. Bryce $\mathbb{R L}$, Stanley F], Gamer ]B. Randomized controlled trial of antenatal social support to prevent preterm birth. Br J Obstet Gynaecol 1991;98:1001 8.

50. Lambe R, Osier C, Franks P. A randomized controlled trial of hypnotherapy for smoking cessation. J Fam Pracr 1986;22:61-5 
51. Hemminki H, Vrta A L, Koponen P. Malin M, Kojo-Austin H, Tuimala R A trial on continuous human support during labor: fetsibility, interventions and mothers" satisfaction. J Psychosom Obstet Gynaecol 1990; 11:239-50.

52. Cogan $\mathrm{R}$, Spinnato $\mathrm{J} A$. Social support during premature labor: effects on labor and the newborn. J psychosom Obstet Gynaecol 1988; $8: 209-16$.

53. Labrie $F$, Candas B, Dupont $A$, Cusan L, Gomez J, Subura RE, et al. Scteening. decreases prostate cance death: first analysis of the 1988 Quebec prospective randomized controlled trial. Prostate $1999 ; 38(2): 83-91$.

54. Cardlund B Randomised, controlled trial of low-dose hepatin for prevention of fatal pulmonary embolism in patients with infectious diseases. The Heparin Prophylaxis Sudy Group. Lancet 1996:347: 1357-61.

55. de lorgeril M, Renaud S, Mamelle N, Salen P, Martin JL, Monjaud I, et al. Mediterranean alpha-linolenic acid-rich diet in secondary prevention of coronary heart disease. Lancet $1994 ; 343: 1454-9$.

56. de Lorgeril M, Salen P, Martin JL, Monjaud I, Delaye J, Mamelle N. Mediterranean diet, tradivional risk factors, and the rate of cardiovascular complications after myocardial infarction: final report of the Lyon Diet Heart Study. Citculation 1999; 99: 779-85.

57. Holmberg L, Bill-Axelson A, Helgesen F, Salo JO, Folmierz P, Haggman M, et al. A randomized trial comparing radical prostatectomy with watch ful waiting in early prostate cancer. N Engl I Med 2002; 347: 781-9.

58. Brus HL, van de Laar MA, Taal E, Rasker J], Wiegman O. Effects of patient education on compliance with basic treatment regimens and health in recent onset active rheumatoid arthritis. Ann Rheum Dis 1998; $57: 146-51$.

59. Goy JJ, Eeckhout E, Burnand B, Vogt P, Stauffer JC, Hurni M, et al. Coronary angioplasty versus left internal mammary artery grafting for isolated proximal left anterior descending artery stenosis. Lancet 1994; 343; 1449-53.

60. Goy J], Eeckhout E, Moret C, Burnand B, Vogt P, Stauffer JC, et al. Five-year outcome in patients with isolated proximal left anterior descending coronary artery stenosis treated by angioplasty of left internal mammary artery grafting. A prospective trial. Circularion 1999; 99:3255-9.

61. MacVicar J, Dobbie G, Owen-Johnstone L, Jagger C, Hopkins M, Kentnedy J. Simulated home delivery in hospital: a randomtsed controlled trial. Br J Obstet Gynaecol 1993; 100:316-23.

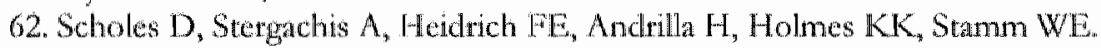
Prevention of pelvic inflammatory disease by screening for cervical chlamydial infection. N Engl J Med 1996; 334: 1362 6.

63. Collins PJ, Larkin EP, Shubsachs AP. Lithium carbonate in chronic schizophrenia: a brief trial of lithium carbonate added to meuroleptics for treatment of resistant schizophrenic patients. Acta Psychiart Scand 1991; 84: 150 4.

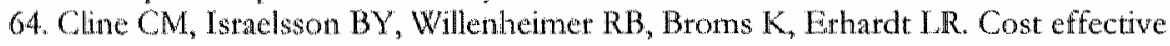
management programme for heart failure reduces hospitalisation. Heart 1998; 80: 442. 6

65. Cherkin DC, Deyo RA, Street JH, Hunt M, Barlow W. Pitfalls of patient education. Limited success of a program for back pain in prinary cate. Spine 1996; 21: 345-55.

66. Dennis M, O'Rourke S, Slattery J, Staniforth T, Warlow C. Evaluation of a stroke family care worker: results of a randomised controlled trial. BMJ 1997; 314: 1071-6.

67. Auvinen A, Vomanen T, Tammela TL, Ala-Opas M, Leppilahti M, Salminen P, et al. A randomized trial of the choice of treatment in prostate cancer: design and baseline characteristics. BJU Int 2001; 88: 708-15 
68. van der Sande R, van Rooijen L, Buskens $\mathrm{B}$, Allart $\mathrm{B}$, Hawhon $\mathrm{K}$, wan der Graf $\mathrm{X}$, et al. Intensive in-patient and commumity intervention versus toutine care after atterapted suicide. A randomised controlled intervention study. Br I Psychatry 1997, 171:35-41.

69. Torgerson DJ, Thomas RE, Campbell MK, Reid DM. Randomized trial of osteoporosis screening. Use of homone replacement therapy and quality-of-life results. Arch Intern Med 1997; 157: 2121-5.

70. Lambert CM, Hurst NP, Forbes JF, Lochhead A, Macleod M, Nuk G. Is day cate equivalent to inpatient care for active theumatoid arthritis Randomised controlled clinical and economic evaluation. BMJ 1998; 316:965-9.

71. Vierhout WP, Knotmerus JA, van OA, Crebolder HF, Pop P, Wesselingh-Megens AM, et al. Effectiveness of joint consultation sessions of general practitioners and orthopaedic surgeons for locomotor-system disorders. Lancet 1995; 346: 990-4.

72. Dey P, Bundred $\mathbb{N}$, Gibbs A, Hopwood P, Baildam A, Boggis C, et al. Costs and benefits of a one stop clinic compared with a dedicated breast clinic: randomised controlled trial. BM] $2002 ; 324: 507$.

73. Zhu SH, Anderson CM, Tedeschi GJ, Rosbrook B, Johnson CE, Byrd M, et al. Evidence of real-world effectiveness of a telephone quitline for smokers. $\mathrm{N}$ Engl J Med 2002; 347: 1087-93.

74. Hux JF, Melady MP, DeBoer D. Confidential prescriber feedback and education to improve antibiotic use in primary care: a controlled trial. CMAJ 1990, 161: 388-92.

75. Hillsdon M, Thorogood M, White I, Foster C. Adwising people to take more exencise is ineffective: a randomized controlled trial of physical activity promotion in primary care. Int I Epidemiol 2002; 31: 808-15.

76. Rodgets H, Atkinson C, Bond S, Suddes M, Dobson R, Curless R. Randomized controlled trial of a comprehensive stroke education program for patients and caregivers. Stroke 1999; 30: 2585-91.

77. Steiner A, Walsh B, Pickering RM, Wiles R, Ward J, Brooking JI. Therapeutic nutsing or unblocking beds? A randomised controlled trial of a post-acute intermediate care unit. BMJ 2001; 322:453-60.

78. Homer CS, Davis GK, Brodie PM, Sheehan A, Barclay LM, Wills J, et al. Collabotation in matemity care: a randomised controlled trial comparing community-based continuity of care with standard hospital care. BJOG 2001; 108: 16-22.

79. Pinto I, Chimeno P, Romo A, Paul L, Haya J, de la Cal MA, et al. Uterine fibroids: uterine artery embolization versus abdominal hysterectomy for treatment-a prospective, randomized, and controlled clinical trial Radiology 2003; 226:425-31.

80. Altman DG, Whitehead J, Pamar MK, Stenning SP, Fayers PM, Machin D.

Randonised consent designs in cancer clinical trials. Eur I Cancer 1995; 31 A: 1934-44.

81. Marquis D. An argument that all prerandomized clinical trials are unethical J Med Philos 1986; 11: $367-83$.

82. World Medical Association. Declaration of Helsinki: recommendations guiding physicians in biomedical research involving human subjects. JAMA 1997; 227: 925-26.

83. Taylor KM, Margolese RG, Soskolne CI. Physicians' reasons for not entering eligible patients in a randomized clinical trial of surgery for breast cancer. N Engl J Mod 1984: 310: $1363-67$

84. Tobias 15 , Houghton J. Is informed consent essential for all chemotherapy studies Fur J Cancer 1994; 30A: $897-99$.

85. Schellings $\mathrm{R}$, Kessels AGH, Geurs R, Sturmans F. IEen herwatrdering wan het Zelen design: de bruikbaarheid bij cen gerandomiseend heroine verstrekkingsexperiment. $T$ Alc Drugs 1995; 21: 67-78.

86. Schellings R, Kessels AGH, Geurs R, Stumans F. Overwegingen bij de opzer van een gerandomiseerd heroine-verstrekkingsexperiment. Tijdschr Soc Gezondheidsz 1996: 376-82. 



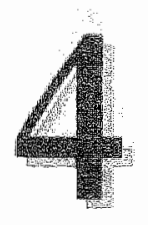

\section{The Zelen design may be the best choice for a heroin-provision experiment}

Ron Schellings ${ }^{1}$, Alphons G.H. Kessels ${ }^{1}$, Gerben ter Riet ${ }^{1}$, Ferd Sturmans ${ }^{2,3}$

1 Department of Epidemiology, Maastricht University, the Netherlands

2 Municipal Public Health Service, Rotterdam, the Netherlands

3 Department of Public Health, Erasmus University Rotterdam, the Netherlands

Journal of Clinical Epidemiology 1999; 52: 503-507. 


\begin{abstract}
Recenty, the Dutch Parliament agreed upon the conduct of a randomized clinical trial on the effects of heroin provision on general health, psychosocial and criminal behavior in long-term addicts. Previous studies failed to establish the effects beyond reasonable doubt. The main reasons why previous trials failed are massive dropout or non-compliance in the control group.
\end{abstract}

Designing a new heroin provision trial, we concluded that the Zelen design provides the best guarantee for obtaining valid study results. Compared with the traditional design, the Zelen design probalbly reduces non-compliance and dropout considerably, thus increasing validity. Depending on the study population, the Zelen design may reduce study precision. However, in a trial aimed at badly integrated addicts the Zelen design can be conducted without loss of precision because baseline measurements will only weakly correlate with effect measurements.

The arguments favoring the Zelen design may be generalized to trials in which the experimental treatment is highly attractive to the study participants. However, the use of the Zelen design precludes blinding of participants who receive the experimental treatment.

We argue that the conduct of studies that predictably tend to produce invalid results is ethically dubious. The ethical problem of studying participants without their consent can be solved by a slight modification of the Zelen design in which the sampling of a control group is postponed. Both the traditional and the Zelen design can imply ethical problems. Both designs can be ethically justifiable and should not be rejected on a priori grounds. 


\section{Introduction}

In agreement with the advice of the Health Council, the Dutch Parliament agreed upon a medical experiment regarding the effects of heroin provision for long-term heroin addicts. ${ }^{12}$ The main hypothesis is: Provision of heroin, in addition to available standard treatment, decreases the harm caused by drug dependence. The outcome measures, in hicrarchical order, will be general health, psychosocial and criminal behavior of long-term heroin addicts. Previous studies suffered particularly from high dropout and non-compliance rates in the control gtoup ${ }^{3-5}$ or did not use a control group. "Therefore, these studies have been insufficiently informative with regard to the effectiveness of heroin provision.

We set out to design an alternative study that would address this pragmatic question and would be less susceptible to dropout and non-compliance while retaining the usual experimental rigor. In the Netherlands, standard treatment for long-term drug addicts is delivered by health services and consists of methadone maintenance and social aid.

\section{The traditional design versus the Zelen design}

Previous trials ${ }^{3-5}$ were conducted as traditional randomized trials in which addicts' consent was obtained before randomization. Addicts who were randomized to a no-heroin provision control group had a clear reason to be disappointed, complied badly, dropped out in large numbers and, in this way, adwersely affected the trials validity. Thus, the challenge is to design a study in which disappointment leading to dismuptive behavior is avoided. In a design that is known as the Zelen design, randomized consent design, or pre-randomization, ${ }^{7.8}$ eligible addicts are randomized, but only in the experimental group is informed consent obtained.

Many investigators reject the use of the Zelen design altogether, claiming that, in general, a number of the randomized participants will refuse the experimental treatment, possibly causing irreparable incomparability of the treatment groups as a result of this potentially selective dropout. ${ }^{10,11}$ Many ethicists reject the Zelen design because control subjects are studied without the latter's consent. We will argue that in a heroin provision experiment, the validity argument may not hold and may even be reversed. The ethical objections may be lessened, and probably reversed, by taking into account the validity argument and by analyzing the meaning of the term study participation in a design that differs from the traditional randomized trial.

\section{Dropout and non-compliance}

Potentially, the most serious threat to the compatability of treatment groups is dropout leading to incomplete data collection and missing data on effect 
mieasurements. $A$ study"s internal validity is reduced if the addicts who drop out are prognostically different from the addicts who complete the study and the dropout rates differ between the treatment groups.

Non-compliance is another factor that may reduce a trial's validity. Addicts may either reject their allocated treatment or may take additional rreatment with an impact on general health, psychosocial or criminal behavior. Both types of noncompliance will alter the treatment contrast in an intention-to-treat analysis.

Traditional design

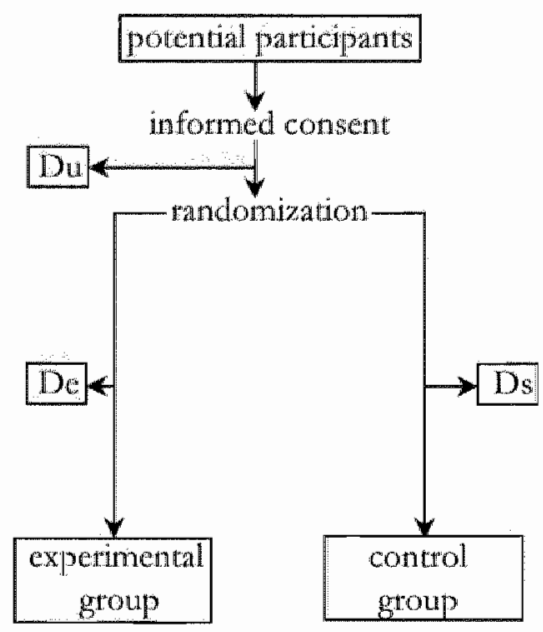

available for effect measurement
Zelen design

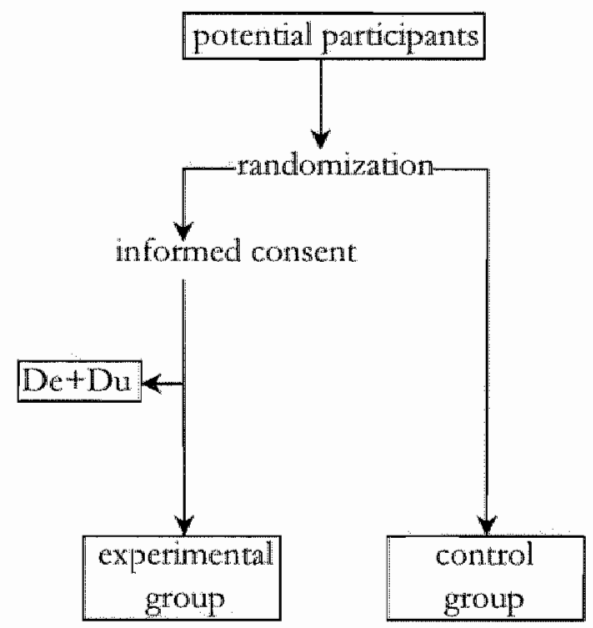

available for effect measurement

Figure 1 Dropout in the traditional design and in the Zelen design.

$\mathrm{Du}=$ unconditional dropout from the trial;

$\mathrm{De}=$ dropout form experimental treatment;

$\mathrm{Ds}=$ dropout from standard treatment.

Figure 1 (lef $h$ ) shows a flow chart of the traditional design. For ease of notation we denote nonparticipation occurring before randomization as a type of dropout. Three types of dropout can be distinguished. First, there is unconditional dropout (Du): addicts who deny informed consent and refuse to participate altogether. Second, there is dropout under the standard treatment condition (Ds): addicts who, after randomization continue to receive normal care without heroin provision (the standard treatment condition), but refuse to participate in effect measurements and other activities that the study protocol may stipulate. Third, is dropout under the experimental treatment condition (De): addicts who reject the heroin provision. As Figure 1 (right) shows, in the Zelen design, dropout occurs in the experimental group only (Du and De), and, as critics correctly noted, only if 
this dropout were strictly random would the validity of the trial be preserved. However, in a heroin-provision trial aimed at long-term addicts, the experimental treatment is so attractive that dropout will most likely be negligible and will not seriously affect the validity of the results. By contrast, in a heroin-provision trial using the traditional design, the fractions Du and De will be small, but dropout in the standard treatment group (Ds) will very likely be substantial. After all, in the traditional design, as soon as the addicts allocated to the control group are informed about their allocation, they will be disappointed. The disappointment may lead to the following problems:

1. The addicts may change their behavior on phenomena that may be the focus of effect measurements (for example, psychosocial functioning). In fact, it is the informed consent procedure combined with (unfortunate) randomization that elicits the disappointment and which should be regarded as an intervention in itself.

2. The addicts may not be willing to participate in the effect measurements at all (Ds).

3. The addicts may become recalcitrant and punish the investigator by providing unreliable answers.

4. The addicts may demand heroin provision and a refusal by the investigational team may lead to annoying behavior at the health services or in adjacent neighborhoods.

The probability that these phenomena occur in the traditional design, after an informed-consent procedure, seems much higher than in the Zelen design.

\section{Validity and precision}

In the preceding section, we atgued that the chances of obtaining valid results in a heroin-provision trial are probably higher with the use of the Zelen design than with the traditional design. However, the use of the Zelen design precludes taking nonstandard measurements in the control group at baseline. In this section, we qualitatively discuss the implications this has for the trial's precision. Note also that the points 1 and 2 in the preceding list are likely to reduce the trial's precision by introducing random error.

The precision depends on the relation between the size of the effect and the corresponding variance. Part of this variance can be eliminated by using the individual before-after differences in the statistical analysis instead of using the last measurement only. A reduction in the variation has the advantage that fewer participants are needed to render a given treatment difference statistically significant; that is, the trial will become more efficient statistically. So, omitting baseline measurements may influence the precision of the trial. 
The choice between the traditional design and the Zelen design depends on weighing the loss of precision due to omitting baseline measurements (Zelen) against the loss of validity due to selective dropout and non-compliance in the control group (traditional). The research question confronting the Dutch Parliament concerns a spectrum of heroin users ranging from moderately societally integrated (as measured by, for instance, the Addiction Severity Index 12) to very poorly integrated addicts. If the trial focused on moderately integrated heroin users, we expect the effects on health and psychosocial behavior to be small. Furthermore, the correlation between baseline and end measurement may be high because of the addicts' relatively stable way of life. In this case, performing a baseline measurement may decrease the variance of the effect measurement and may be very useful. Furthermore, the likelihood of dropout and non-compliance is limited, resulting in a fair chance of valid results. Both aspects could favor the traditional design.

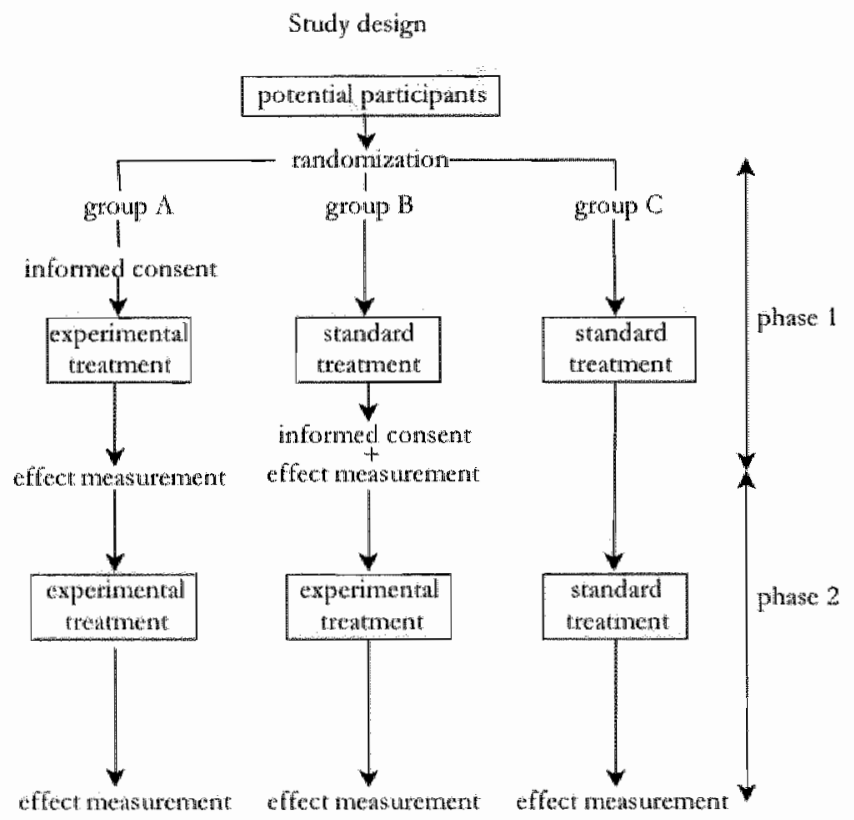

Figure 2. Study design for a randomized heroin-provision experiment.

By contrast, for badly integrated heroin users, the circumstances are much more unstable. We expect the correlation between baseline and end measurements to be low, whereas the effect on health and psychosocial behavior might be fairly large. Moreover, dropout and non-compliance due to the disappointment of allocation to the standard group will be a greater problem in these addicts compared to the 
better integtated ones. Consequenty, if the study population consists of the badly integrated heroin users, the Zelen design seens preferable. Until now, the Dutch Parliament has focused on the older addicts, with a long history of heroin abuse, who are in bad health and whose level of psychosocial functioning is low.

\section{A heroin provision experiment in badly integrated addicts}

The attractiveness of the intervention implies that many heroin addicts will volunteer for heroin provision. So, in the rraditional design, the addicts in the control group will be frustrated after being tefused the desired intervention. Theoretically, in the Zelen design there will be no frustration; however, in practice some addicts in the control group, being aware of the experiment, may demand participation in the provision group, which will then be denied. This may cause disappointment and some of the problems already described here. It should be much easier, however, to explain to disappointed addicts that only about $10 \%$ of all addicts were selected for the experiment than it will be to pacify the unfortunate control addicts who were confronted with a $50 \%$ expectation of heroin-provision as would occur in the informed-consent procedure of any traditional design using a fixed allocation tatio of $50 \%$. Furthermore, if the population from which the experimental group is sampled is large compared with the size of the trial, not every addict in the (future) control group may be aware of the experiment. By contrast, in the traditional design, every addict in the control group is alerted by the informed-consent procedure.

To limit the disruption of the drug scene to a brief time interval, we propose to select potential addicts at one time using data routinely collected for health care evaluation purposes. As far as relevant, these data can also be used as baseline values (age, gender, duration and type of drug use, etc.). For the effect measurements concerning health and psychosocial behavior, a personal appointment with each addict is necessary. A design that minimizes dtopout and non-compliance and maximizes the likelihood of a full comparison between the experimental group and the control group is presented in Figure 2.

In this design, addicts are randomized to three groups, and the study period is divided into two phases of 6 months each. In group A, the experimental group, informed consent is obtained. This group will receive heroin in addition to standard treatment during both phases. Group B is a control group in which informed consent will not be obtained until just before the end of phase 1 , and in phase 1, these addicts receive standard treatment as usual. At the end of phase 1, the effects on health and psychosocial behavior in group $A$ will be compared with those in group B. To obtain the necessary data for that comparison in group $B$, just before the end of phase 1 group $B$ is offered heroin provision during phase 2 . We assume that almost all addicrs will given their consent because of the 
atractweness of reciving heroin Group $C$ is a control group for the effects on criminal behavior dung phase 1 and phase 2. In this group, personal encounters with the addicts ate not necessary for the effect measurement, and no informed consent wll be asked. At the end of the follow-up period or shortly thereafter, the investigators will provide police and court authorities with the names of the addicts belonging to groups $A, B$, and $C$. The authorities will link these names to their filles of criminal activities. Finally, the authonities will report back to the investigators only descriptive criminal statistics (means, standard deviations, etc.) on the group level $(A, B$ and $C$ ) within the follow up period. Because only a small percentage of the criminal activities are registered in police and justice records, control group $C$ needs to be larger than the other groups. In addition, group $C$ will be followed longer.

Phase 1 is the most important phase of the study, as a compatison of the effects on health and psychosocial behavior can be measured at the end of it. Phase 2 serves different purposes. First, the effects on criminal activities will be measured at the end of phase 2. Second, at the end of phase 2 , it is possible to perform follow-up measurements regarcling health and psychosocial behavior in groups $A$ and $B$ to determine the stability of the effects, if any. Finally, the results of the statistical analyses of phase 1 , and policy decisions based on these tesults, can be awailable halfway through phase 2 . So, a time-gap in the care for addicts who participated in the trial can be avoided.

\section{Ethilcal considerations}

In clinical medicine, both the Zelen design and the traditional design have been the subject of ethical scrutiny and controversy. In both designs the physician-patient relationship can be adversely affected because of the physician's preference for one of the treaments." However, in the raditional design, this relationship may be under even greater pressure because patients know that treatment allocation is purely a matter of chance. ${ }^{14}$ In addition, sometimes, complex information must be given about the treatment options and procedures. There are indications that patients cannot absorb all important information given in an informed-consent procedute. 15 Thus, the objective of an informed-consent procedure is often imperfectly realized. ${ }^{16}$ In the Zelen design no consent is obtained in the control group, and some ethicists questioned to what extent data can be used without informing this group. ${ }^{17}$ However, as Zelen noted, this does not differ from the use of aggregated data routinely collected in registries at clinical departments and care facilities. 18

In discussions with colleagues and ethicists we have noticed that the Zelen design may arouse strong emotions, sometimes to the extent that reflective thinking is replaced by reflexive denunciation and rejection. We hope that our 
essay is convincing that there are strong validity arguments in favor of the $Z$ elen design in a hetoin provision trial. Note that methodological rigor and ethics are related issues. In this field of study, it can hardy be ethically jusufiable to reemploy the previously used designs, which have been repeatedly shown to yield results that are probably invalid but at any rate unconvincing. 3 .6. We must balance the methodological advantages against the disadvantage that we cannot inform the control group about their apparent participation. It is tempting, however, to define "study participation" as "being subject to follow-up from the moment of randomization." Although this definition may apply in the traditional randomized trial, other study designs, whether experimental or nonexpetimental, may actually use or requite other definitions. Consider an example from nonexperimental epidemiological studies. Using a questionnaire, an investigator collects data on anthypertensive drug use in a group of people. After the data collection is over, the idea of a control group emerges. The investigator decides to use general population data with which to compare his or her data. Should we conclude that the general population patticipated in the study from the very start What if a fandom sample from the general population was asked also to complete the investigator"s questionnaire; from which point in time would this sample be participating in the study?

Returning to the heroin provision trial, Figure 3 shows the outline of an alternative study design in which, in several cities, all eligible heroin addicts (for example, badly integrated ones) in the care provider's files are identified. One could imagine that in some cities, far more eligible addicts are identified than needed for the trial. So, of all eligible addicts, a random sample will teceive, after giving informed consent, heroin provision during phase 1 . At the end of phase 1 , a second random sample is taken from the group of eligible addicts who were not included in the first sample. All addicts in the second sample will be traced and asked for theil. consent to participate in phase 2 , in which they will receive heroin provision after a baseline measurement. The baseline measurements in the second sample will bo used for comparison with the measurements at the end of phase 1 in the first sample. We would argue that the second sample (control group) does not participate in the study unt the moment they consent to bascline measurements and heroin provision. In fact, that there may be more eligible addicts availables than needed is not crucial to the argument, but for some, this may enhance the idea of sampling only a fraction for inclusion in the tral. It struck us that some colleagues and ethicists rejected our plan for a heroin trial when we presented it as a Zclcon design, whereas they judged the plan as elegant when we presented it as shown in Figure 3. This might imply that the chances of support and funding of projects that require a Zelen concept may increase if presented as we did here. 


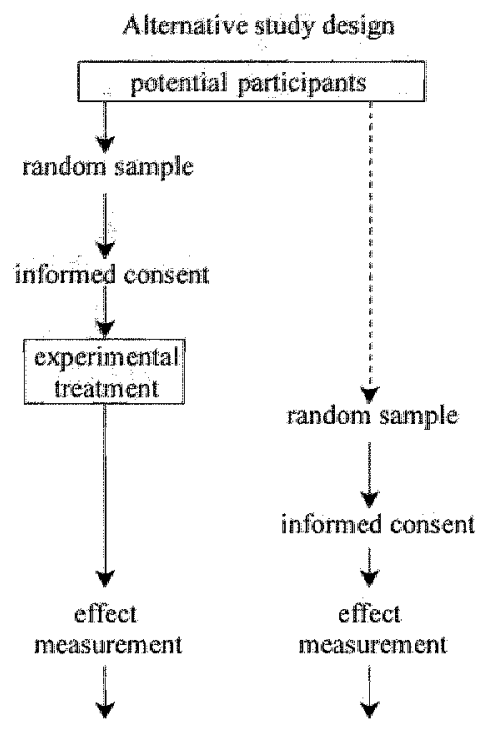

Figure 3. Alternative study design. Broken lwe denotes standard care.

\section{Conclusion}

We have focused on the use of the Zelen design in a heroin-provision experiment in badly integrated long-term addicts. However, we think that our design considerations have a wider applicability. In this final section, we describe these considerations more generally.

The Zelen design as an alternative for the traditional randomized design should be considered if: (a) the experimental intervention is highly attractive for all potential participants, and (b) the control group will receive standard treatment. If these conditions are met, the choice between the two designs depends on weighing the loss of validity due to selective dropout and non-compliance in the control group against the potential loss of precision due to not performing a baseline measurement. In most situations, exact numerical data on the correlation between baseline and end measurements will not be available. The use of the Zelen design precludes blinding of participants who receive the experimental treatment. Sometimes, the traditional as well as the Zelen design involve ethical problems. In our opinion, both designs are ethically justifiable and should not be rejected on a priori grounds. 


\section{References}

1. The drugs policy of the Netherlands; Continuity and change. Rijswijk: Ministry of Foreign Affairs, Ministry of Health, Welfare and Sports, Ministry of Justice, Minstry of the Interior; October 1995.

2. The prescription of heroin to heroin addicts; Sub-report from a conminte of the Health Council of the Netherlands. The Hague: The Winister of Health Weltate and Sports; June 1995. No. 1995/12E.

3. Hartnoll RT, Mitcheson MC, Battersby $A$, Brown $G$, Ellis M, Fleming $P$, et al.

Evaluation of hetoin maintenance in controlled trial. Arch Gen Psychatry 1980; 37 : $877-84$.

4. Wchtenhagen A. Diversifizierte Verschreibung von Berabungsmitteln an Heroinabhängige: Grundlagen, Versuchsplan, Begleitforschung. Schweiz Rundsch Med Prax 1994; 83: 931-6.

5. Uchtenhagen A, Gutzwiller F, Dobler-Mikola A, Blattler R. Versuche für cine ärztiche Verschreibung von Betäubungsmitteln: Zwischenbericht der Forschungsbeauftagten. Zürich: Institut für Suchtforschung in Verbindung mit der Universität Zürich \& Institur für Sozial und Präventivmedizin der Universität Zürich: 1995.

6. Marks J. Das Englische System in Widnes, Merseyside. Heroinvergabe. In: Neumeyer J, Schaich-Walch $G$, Eds. Zwischen Legalisienung und Normalisiering. Marburg: Schüren Presseverlag; 1992: 56-64.

7. Zelen M. A new design for randomized clinical trials. New Engl J Med 1979; 300: 12425.

8. Zelen M. Randomized consent designs for clinical trials; an update Stat Med 1990; 9: 645-6.

9. Ellenberg SS. Randomization designs in comparative clinical trials. New Engl J Med 1984; 310: 1404-08.

10. Anbar $\mathrm{D}$. The relative efficiency of Zelen's prefandomization design for clinical trials. Biometrics 1983; 39: 711-8.

11. Matts J, McFugh R. Randomization and efficiency in Zelen"s single-consent design. Biometrics 1987; 43: 885-94.

12. Mclellan AT, Luborsky L, Woody GE, O'Brien CP. An improved diagnostic evaluation instrument fot substance abuse patients: The Addiction Severity Index.] Nerv Ment Dis 1980; 168: 26-33.

13. Sikerman WA, Altman DG. Parients' preference and randomised trials. Lancet 1996, 347: $171-4$.

14. Taylor KM, Margolese RG, Sokolne CL. Physicians' neasons for not entering cligible patients in a randomized clinical trial of surgery for breast cancer. New Engl J Med 1984:310: 1363-7.

15. Byme DJ, Napier A, Cuschieri A. How informed is signed consent BM] 1988; 296: $839-40$.

16. Cassileth BR, Zupkis RV, Sutton-Smich K, March V. Informed consent: why arc its goals imperfectly realized? New Engl J Med 1980; 302: 896-900.

17. Beauchamp TL, Cook RR, Fayerweather WE, Rabe GK, Thar WE, Cowles SR, Spivey GH. Ethical guidelines for epidemiologists. J Clin Epidemiol 1991; 44: 151\$-169S.

18. Zelen M. Randomised consent trials. Lancet 1992; 240 . 375. 



\section{Members of research ethics}

\section{committees accepted a modification of the randomized consent design}

Ron Schellings ${ }^{1}$, Alfons $G$ Kessels ${ }^{2}$, Gerben ter Riet ${ }^{3,4}$, Jos Kleijnen ${ }^{5}$, Pieter Leffers ${ }^{6}, J$ André Knottnerus ${ }^{7}$, Ferd Sturmans ${ }^{8}$

1 Public Health Supervisory Service of the Netherlands, the Health Care Inspectorate, The Haguc, the Netherlands

2 Department of Clinical Epidemiology and Medical Technology Assessment, University hospital Maastricht, the Netherlands

3 Academic Medical Center, Department of General Practice, Amsterdam, the Netherlands

4 Horten Centre, Zurich University, Switzerland

5 Centre for Reviews and Dissemination, University of York, United Kingdom

6 Department of Epidemiology, Maastrichr University, the Netherlands

7 Department of General Practice, Maastricht University, the Netherlands

8 Department of Public Health, Erasmus University Rotterdam, the: Netherlands

Journal of Clinical Epidemiology 2005; 58: 589-594 


\begin{abstract}
Objective The use of randomized consent designs has been subject of methodological and ethical controversy. In most Western countries, research ethics committees make the decision whether a randomized consent design can be applied. The purpose of the study is to assess to what extent a randomized consent design and a modification of this design is accepted by research ethics committees, in terms of ethics, health law and methodology.
\end{abstract}

Study design and setting $\mathbb{A}$ postal survey was conducted among members of research ethics committees in the United Kingdom, and in the Netherlands, with professional competence in ethics, (health) law, methodology or clinical practice.

Results In both the UK and in the Netherlands, the modified tandomized consent design appears to be statistically significantly more acceptable than the randomized consent design, with respect to ethical and judicial aspects. The overall rejection ratte of the randomized consent design was $66 \%$ in the UK and $59 \%$ in the Netherlands. However, the modified randomized consent design was rejected by $47 \%$ and $41 \%$ in the two countries, respectively.

Conclusion the modified randomized consent design appears to be more acceptable than the randomized consent design. To increase consistency in the way research ethics committees handle study protocols, a discussion about the use of randomized consent designs appears necessary. 


\section{Introduction}

In 1977, Marvin Zelen proposed a randomized consent design in which eligible patients are randomized and informed consent is sought only in those who are assigned to the experimental group. ${ }^{1}$ Normally consent is sought from all eligible patients befote random treatment assignment. However, physicians often have difficulty to invite patients to participate in clinical trials because of anticipated problems with obtaining informed consent. In Zelen's randomized consent design this problem is avoided. 1.2 A serious methodological drawback of this Zelen design is that withdrawal from the experimental group after the informed consent procedure may bias the estimate of the true treatment effect. ${ }^{3-5}$ In addition, many ethicists and health lawyers opposed the design arguing that the participants are randomized without prior consent, and that those in the reference group are not even aware of their involvement in a study.6,7 To meet this concetn a variant of the randomized consent design was introduced in which informed consent is sought in both treatment arms after randomization, $2,8-11$

Today the use of a randomized consent design is still controversial. For example, Zelen's consent design is not acceptable to the British Medical Research Council and current US federal guidelines also preclude its use, ${ }^{3,12}$ In recent years, a $\mathrm{BMJ}$ debate about informed consent that included ethical problems in using randomized consent designs, roused the emotions ${ }^{13-17}$ and authors have summarized the pros and cons of these designs. ${ }^{18-19}$ Also in the Netherlands, opinions differ strongly on the use of the Zelen or pre-randomization design. ${ }^{20}$ Again, the most prominent counter-argument was that participants are not aware of their entry into a study or to be randomized. To refute this argument, a modified randomized consent design was proposed in which the reference group members are sampled at the end of the study period allowing them to enter the study after giving consent (see Figure 1 b). In this way, the ethical objection that the reference group members were not aware of being included in a study should be met without losing the methodological advantage of the randomized consent design where attractive treatments are concerned ${ }^{21}$ (for more details see the method section).

The main purpose of the present study is to compare a randomized consent design to the proposed modification thereof with respect to their acceptability in terms of ethics, health law and methodology. In addition, we wanted to examine whether differences in acceptability exist between the United Kingclom and the Netherlands, which differ greatly with respect to their statutory regulations concerning medical experiments. 


\section{Methods}

\section{Context}

In most Western countries, research ethics committees consisting of members with professional competence in ethics, health law, methodology, or clinical practice make the decision whether a randomized consent design can be applied. However, between countries, the statutory regulations vary widely. For example, in the Netherlands, the procedure of teviewing health related research proposals has been standardized in the Medical Research Involving Human Subjects Act (WMO). 22 This act stipulates that any reseatch ethics committee should include an ethicist, a (health) lawyer, a methodologist and a clinician. In the United Kingdom, there is no law comparable with the Dutch WMO and arrangements for reviewing the ethics of local and multi-center research are described in Health Service Guidelines of the National Health Service (NHS). ${ }^{23}, 24$ The central office for tesearch ethics committees lays down rules for the composition of a research ethics committee. ${ }^{25}$ A committee should contain a mixture of experts and lay members. The expert members should have expertise in methodology, ethics, clinical practice or in statistics relevant to research or pharmacy. At least one third of the members shall be laypersons.

\section{Study design}

In November 2000 , we mailed questionnaires to 240 traceable local research ethics committees (LREC) and multicenter research ethics committees (MREC) in the United Kingdom, including the central office for research ethics committees (COREC). In the Netherlands, we mailed the questionnaires to the 77 registered medical ethics committees (local research ethics committees), and to the central medical ethics committee (CCMO). The questionnaire described a fictitious study on the provision of heroin to longterm heroin addicts, which met three preconditions for the proper use of a tandomized consent design ${ }^{21}$, namely, 1) the experimental intervention cannot be blinded and is very attractive (in which situarion full prior consent would evoke contamination, and differential noncompliance and dropout in the reference group); 2) no personal measurement(s) ate necessary until informed consent is obtained; and finally, 3) the reference group receives standard treatment (usual care). A randomized consent design with informed consent for effect measurements in the reference group, indicated as randomized consent design (Figure 1a) and the modified version, indicated as modified randomized consent design (Figure 1b), were explained as feasible design options for this study. 


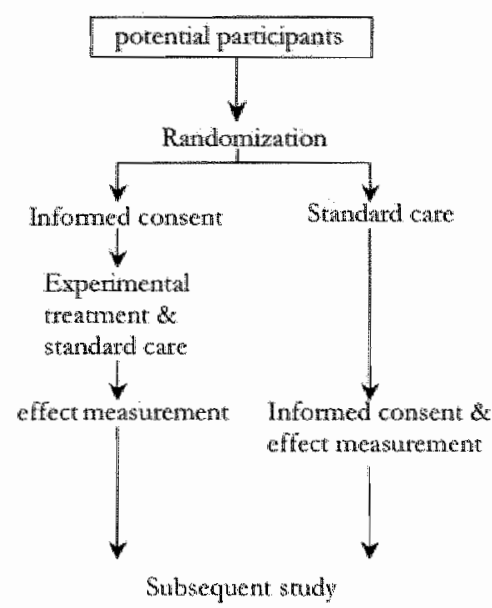

Figure 1a. Randomized consent design

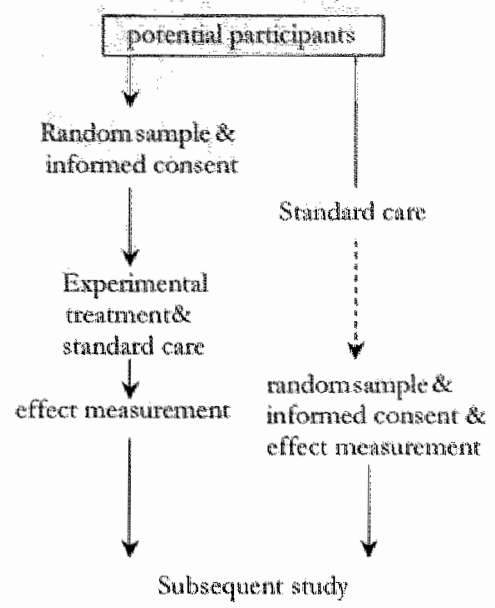

Figure $1 \mathrm{~b}$. Modified randomized consent design

In the randomized consent design, addicts are randomized to two groups. In the experimental group informed consent is sought after randomization. This group receive heroin in addition to standard treatment. The addicts in the reference group receive standard treatment. Therefore, at the end of the study the effects on health and psychosocial behavior in the experimental group would be compared with those in the reference group. At the end of the study, the reference group would be asked for their consent to participate in a subsequent study in which they will receive heroin in addition to standard treatment. We expected that almost all addicts would give their consent because of the attractiveness of receiving heroin. The baseline measurements for the subsequent study will be used as the effect measurement for the first study.

In the modified randomized consent design, all eligible hetoin addicts in the care providers' files are identified. A random sample will receive, after having given informed consent, heroin in addition to standard treatment. At the end of the study period a second random sample is taken from the original group of the eligible addicts who were not included in the first sample. All addicts in the second sample will be extensivelly traced and asked for their consent to participate in a subsequent study in which they will receive heroin in addition to standard care.

The questionnaire consisted of questions about ethical, judicial and methodological acceptability of the randomized consent design and the modified randomized consent design on a three-point scale: acceptable, not acceptable, do not know. Furthermore, the participants wete asked to indicate their preference for one of the designs from an ethical, judicial and methodological viewpoint and an opportunity was offered to give short comments. Through the secretary of the 
research ethics committees, four members of each of the 240 ethics committees in the UK and the 78 ethics committees in the Netherlands with professional competence in ethics, (health) law, methodology or clinical practice, were asked to fill in the questionnaire. All the committees received a reminder after 6 to 8 weeks, including a second copy of the questionnaire. We presented our study protocol to the research ethics committee of the Maastricht University hospital, which concluded that no formal approval was required as it concerned a postal survey.

\section{Statistical Analysis}

Proportions between groups were compared with a Chi-square test and the comparison of proportions for paired data were made using McNemar's test. Equality of proportions was tested with a binomial distribution. To test null hypotheses a significance level of 0.05 (two-sided) was used.

\section{Results}

With four participants in each committee the maximum number of participants that could be achieved in the United Kingdom was 960. However, the questionnaires for 9 committees were undeliverable and were returned by post and 2 committees had merged, leaving 920 potential participants of which $337(37 \%)$ responded. Of these, 11 responders did not cross off any of the questions, leaving $326(35 \%)$ analyzable responses. In the Netherlands, the maximum number of participants was 312. However, 14 committees registered at the central medical ethics committee had not judged any study protocol in the year 2000 and we assume that these committees were not operational. Of the 256 potential participants, $136(53 \%)$ responded. Here, three responders were excluded from the analysis because they completed only one or two answers, leaving 133 (52\%) responders.

Table 1 presents the acceptability of the randomized consent design and the modified randomized consent design to responders in the United Kingdom and in the Netherlands with respect to the ethical, judicial and methodological aspects. In the UK and in the Netherlands the modified randomized consent design appeared to be statistically significantly mote acceptable than the randomized consent design, with respect to ethical and judicial aspects. In the UK, the methodological acceptability of the randomized consent design is statistically significantly greater than the acceptability of the modified version. 
Table 1. Ethical, judicial and methodological acceptability and overall tejection of the randomized consent design and a modified malomized consent design monom 459 members of British and Durch research ethics commitees.

\begin{tabular}{|c|c|c|c|}
\hline Unted KNom down $(\mathrm{N}=326)$ & $\begin{array}{l}\text { Ranclomizad } \\
\text { consent design } \\
\mathrm{N}(\%)\end{array}$ & $\begin{array}{l}\text { Modified randomised } \\
\text { consent design } \\
N(\%)\end{array}$ & $\begin{array}{l}\text { P-value of } \\
\text { the difference }\end{array}$ \\
\hline Ethical acceptability & $111(34)+$ & $189(58)$ & $P<0.0001$ \\
\hline Judicial acceptability & $86(26)$ & $115(35)$ & $P<0,0001$ \\
\hline $\begin{array}{l}\text { Methodological } \\
\text { accepcability }\end{array}$ & $165(51)$ & $138(42)$ & $P=0.006$ \\
\hline \multicolumn{4}{|l|}{ The Nedberlands $(\mathrm{N}=133)$} \\
\hline Ethical acceptability & $61(46)$ & $82 \quad(62)$ & $p<0.0001$ \\
\hline Judicial acceptability & $45(34)$ & $69(52)$ & $p<0.0001$ \\
\hline $\begin{array}{l}\text { Methodological } \\
\text { acceptability }\end{array}$ & $56(42)$ & $47 \quad(35)$ & $\mathrm{P}=0.10$ \\
\hline \multicolumn{4}{|l|}{ Overall regection +} \\
\hline United Kingdom $(N=326)$ & $216 \quad(66)$ & $152(47)$ & $p<0.0001$ \\
\hline The Netherlands $(N=133)$ & 78 (59) & 55 (41) & $\mathrm{P}=0.001$ \\
\hline
\end{tabular}

* McNennar's test.

t As both designs could be acceptable with respect to one of the aspects, the sum could exceed the total number of responders.

t The overall rejection is determined if a responder rejected the design with respect to at least one of the three aspects (ethical, judicial, methodological).

To determine an overall rejection rate, we assumed that the research protocol under a committee's review would be rejected if a responder rejected the design with respect to at least one of the three aspects. For both designs, the overall rejection is higher in the UK than in the Netherlands, but the differences are not: statistically significant.

Table 2 presents the preferences for the randomized consent design or the modified consent design in responders who accepted both designs with respect to the ethical, judicial and methodological aspects. The preference of the modified design is higher than that of the randomized consent design, but not to a statistically significant degree. 
Table 2. Distribution of preferences for the randomized consent design or a modified tandomized consent design among members of British and Dutch ethics committees who deemed both designs acceptable.

\begin{tabular}{|c|c|c|c|c|}
\hline & $\begin{array}{l}\text { Both designis } \\
\text { acceptable } \\
\mathrm{N}\end{array}$ & $\begin{array}{l}\text { Preference } \\
\text { randomized } \\
\text { consent design } \\
\mathrm{N}(\%)\end{array}$ & $\begin{array}{l}\text { Preference modified } \\
\text { randomized } \\
\text { consent design } \\
\mathrm{N}(\%)\end{array}$ & $\begin{array}{l}\text { p-value of } \\
\text { the } \\
\text { difference* }\end{array}$ \\
\hline \multicolumn{5}{|c|}{ Uniled Kingdom $(N=326)$} \\
\hline Ethical & 90 & $33(37)$ & $22(24)$ & $P=0.18$ \\
\hline Judicial & 74 & $15(20)$ & $20(27)$ & $\mathrm{P}=0.50$ \\
\hline Methodological & 107 & $45(42)$ & $28(26)$ & $p=0.06$ \\
\hline \multicolumn{5}{|c|}{ The Netherlands $(N=133)$} \\
\hline Ethical & 58 & $14(24)$ & $27(47)$ & $P=0.06$ \\
\hline Judiciall & 42 & $11(26)$ & $17(40)$ & $\mathrm{P}=0.34$ \\
\hline Methodological & 39 & $12(31)$ & $17(44)$ & $\mathrm{P}=0.46$ \\
\hline
\end{tabular}

* Equality of numbers was tested with a binomial distribution.

In table 3 remarks are summarized of $154(47 \%)$ British and $71(53 \%)$ Dutch responders, who used the opportunity in the questionnaire to give comments.

Table 3. Summary of comments of $154(47 \%)$ British and $64(48 \%)$ Durch responders.

\section{Remarks *}

- The information to make an informed judgment, (especially for real assessment of ethical aspects) is insufficient $(12,4)$.

- Irrespective of the design, the control group receiving standard care has the right to know and consent to their participation in research $(39,13)$.

- In the randomized single consent design the control group receiving standard care has the tight to know and consent to their participation in research $(24,4)$.

- Both designs appear to contravene the General Medical Council Guidelines or the WMO (3, 4).

- The randomized single consent design appears to contravene the General Medical Council Guidelines of the WMO $(3,2)$.

- Provision of heroin in the intervention group is an inducement to the informed consent and therefore not correct $(10,6)$.

- Provision of heroin to the controls after the study could be perceived as a reward for having unknowingly given up their right to be informed $(4,5)$.

- It is uncthical to provide a (dangerous) drug like heroin in a trial $(13,1)$.

- The effect measurement is not reliable because participants are under the influence of the narcotic drug $(3,0)$.

- In the modified tandomized consent design there will be problems with tracing the eligible pattients in the concrol group after the study to participate in the subsequent study $(8,3)$.

- The results of the study will be biased because in the modified consent design study groups ate selected differently, informed consent is required at different times $(18,11)$.

- Resenther has access to confidential information without consent of the study participants $(11,1)$.

- There is no difference benween the two desigus and they are equal in terms of ethics, law and methodology. $(12,10)$.

* The numbers of British and Dutch responders that made that specific remark, respectively, are indicated in parentheses. 


\section{Discussion}

The overall rejection rate of the randomired consent design by members of research ethics committees was $66 \%$ in the UK and $59 \%$ in the Netherlands. The modified randomized consent design was rejected with $47 \%$ and $41 \%$, respectively. This suggests that the modified version of the design is mote acceptable than the randomized consent design. In particular, the modified randomized consent design appears to be more acceptable than the tandomized consent design with respect to ethical and judicial aspects. In both countries, the differences between the acceptability of the designs are highly statistically significant.

Our study has several limitations. The response rates were low $(37 \%$ and $53 \%$ in the UK and the Netherlands, tespectively). In a comparable UK postal study the response rate was $51 \%$ and this is consistent with many postal surveys. ${ }^{26}$ In another UK postal study the authors could not calculate the response rate because of the vatiation in the number of local research ethics committees during one year. ${ }^{27}$ In our study, we did not find one obvious explanation for the low response tate. Some responders complained that the central office for research ethics committees had not formally approved the study. Furthermore, in the UK, the composition of committees is not regulated by law and therefore it is not clear whethet all four competencies were present. Unfamiliarity with the randomized consent design and the description of the fictitious study that may have been too condensed may have caused non-response. Finally, as indicated by several responders' remarks, our example of a very attractive intervention, i.e. the provision of heroin, might have triggered negative emotion and may have precluded members of commitrees to participate. Nevertheless, the results of our study are an indication of how a substantial proportion of participants judges about the acceptability of the presented study designs.

As far as we know, this is the first study on the acceptability of specific designs or study protocolls among research ethics committee members. Thercfore, we have no reference for our findings. Although a lot of comments (see table 3) concerned the provision of heroin as an unacceptable intervention, we will not discuss this item, as the study protocol was only a vehicle to demonstrate the use of the tandomized consent design.

We think that the patients in the reference group would undergo the same procedures in the randomized consent design as in the modified consent design. This is also indicated by some of the responders in their remarks. So, if there are ethical or judicial objections because of infringement on the rights of the participating patients, they hold for the tandomized consent design as well as for the modified design. Furthermore, regardless whether the randomized consent design or the modified version is used, the interpretation of the results would be identical. From this we concluded that there is no essential difference between the two designs with respect to ethical, judicial or methodological aspects. The 
difference is only on paper. Consequendy, in our opinion, both designs should be equally acceptable. However, in this study a statistically significandy higher proportion of responders rejected the randomized consent design than did the modified tandomized consent design. "The increased acceptability of the modified design is also shown in the comparisons of the acceptability on ethical and judicial grounds. This suggests that ethical and judicial arguments play an important part in the decision whether to accept the randomized consent design ot the modified randomized consent design. Anyway, the results of our survey show various points of view with respect to the acceptability of the randomized consent design or the modified version.

To increase consistency in the way research ethics committees handle study protocols, an open discussion about the pros and cons of the use of randomized consent designs is important. Our survey may be instrumental in achieving such discussion leading to consensus, for instance by means of a Delphi consensus technique 28 or to reach another form of understanding, in compliance with the ethical principles of the Declaration of Helsinki. ${ }^{29}$ This would be very important since, apart from ethical acceptability per se, it would not be a satisfactory situation if investigators would formulate essentially similar study designs in a different way for the mere purpose of making a better chance for ethical approval. 


\section{References}

1. Zelen M. Staditical options in clinical trials. Semin Oncol 1977,4.4416.

2. Zelen M. A new design for randomized clinical trials. New Engl J Med 1979; 300:1242. 5.

3. Altman DG, Whitehead J, Parmar MKB, Stenning SP, Fayers PM, Machin D. Randomised consent designs in cancer clinical trials. Eur J Cancer 1995; 31A: 193444 .

4. Anbar $D$. The relative efficiency of Zelen's pretandomization design for clinical trals. Biometrics 1983; 39:711-8.

5. Matts J, McHugh R. Randomization and efficiency in Zelen's single-consent design. Biometrics 1987; 43: 885-94.

6. Ellenberg $\$ S$. Randomization designs in compantive clinical trials. New Engl] Med 1984; 310: 1404-08.

7. Beauchamp TL, Cook RR, Fayerweathet WE, Rabe GK, That WE, Cowles SR, Spivey GH. Ethical guidelines for epidemiologists. J Clin Epidemiol 1991; 44: $151 \mathrm{~S}-69 \mathrm{~S}$.

8. Zelen M. Alternatives to classic tandomized trials. Surg Clin N Am 1981; 61: 1425-32.

9. Zelen M. Straregy and altemate randomized designs in cancet clinical trials. Cancer Treat Rep 1982; 66: 1095-1100.

10. Zelen M. Randomized consent designs for clinical trials: an update. Stat Med 1990; 9 ; 645-46.

11. Knotmetus ]A. Health care research in ambulatory care. In: Ethick en recht in de gezondheidszorg (Dutch language). Deventer: Kluwer; 1997. p. TV 151-94.

12. Zelen M. Authors"reply [letter to the editor]. Stat Med 2002; 21: $2601-05$.

13. Smith R. Informed consent: the intricacies. BMJ 1997; 314: 1059-60.

14. Informed consent in medical research [letters]. BMJ 1997; 314: 14707-83.

15. Informed consent [letters]. BM] 1997; 315:247-54.

16. Torgerson DJ, Roland M. What is Zelen's design? BMJ 1998; 316:606.

17. Smith R. Informed consent: edging forward (and backwardsj. BMJ 1998; $316: 949.51$.

18. Homer CS. Using the Zelen design in randomized controlled trials: debates and controversies. J Adv Nurs 2002; 38: 200-7.

19. Palmer CR. Ethics, data-dependenc designs, and the strategy of clinical trials: time to start learning-as-we-go? Stat Methods Med Res 2002; 11: 381-402.

20. Gezondheidstaad. Informed consent en prerandomisatie. Den Hagg: Gezondheidsraad, 1999.

21. Schellings R, Kessels $A G H$, Rier ter $G$, Sturmans F, "The Zelen destgn may be the best choice for a heroinmproxision experiment. J Clin Epidemiol 1999; 52.50307 .

22. Ministry of Health, Welfare and Sports. WMO. The Medical Research Involving Human Subjects Act. The Hague: Ministry of Health, Welfare and Sports, 1998.

23. Department of Health. Local Research Ethics Committees. London: DoH, 1991. (MSG(91)5.).

24. Department of Health. Ethics committe review of multi-centre research. Jondon: $\mathrm{DoH}, 1997 .(\mathrm{HSG}(97) 23$ )

25. Centrall Office for Research Ethics Committees (COREC). Governance artangenents for NHS Research Ethics Committees. London: Central Office for Research Ethics Committees, 2001.

26. Osborn DPJ, Fulford KWM. Psychiatric tesearch: what ethical concerns do LRECs encounter? A postal survey. I Med Ethics 2003; 29: 55-6.

27. Godfrey $E$, Wray E, Nicholson R. Another look at LREC annual reports. Bull Med Eth $2001 ; 171: 13-21$. 
28. Delbecq $\Lambda$, Ven $A H$ van de, Gustafson $D H$. Group techniques for program planning; a guide to nominal group and Delphi processes. Glenwew: Scott, Foresman, 1975.

29. World Medical Association Declaration of Helsinki. Ethical principles for medical research involving human subjects. J AMA 2000; 284: 3043-5. 


\section{Indications and requirements for the use of pre-randomization}

Ron Schellings ${ }^{1}$, Alfons G Kessels ${ }^{2}$, Gerben ter Riet ${ }^{3}{ }^{4}$, Ferd Sturmans ${ }^{5}$, Guy A. Widdershoven ${ }^{6}, \mathrm{~J}$ André Knottnerus ${ }^{7}$

1 Public Health Supervisory Service of the Netherlands, the Health Care Inspectorate, The Hague, the Netherlands

2 Department of Clinical Epidemiology and Medical Technology Assessment, University hospital Maastricht, the Netherlands

3 Academic Medical Center, Department of General Practice, Amsterdam, the Netherlands

4 Horten Centre, Zürich University, Switzerland

5 Department of Public Health, Erasmus University Rotterdam, the Netherlands

6 Department of Health Ethics and Philosophy, Maastricht University, the Netherlands

7 Department of General Practice, Maastricht University, the Netherlands

Submitted for publication 


\begin{abstract}
Background Athough in effectiveness studies the conventional randomized trial, in which informed consent is obtaned in all participants before randomization, is the first choice, this design is not the panacea for all research questions. "To counter contamination problems pre-tandomization designs might be an altemative, Pre-tandomization implies that the randomization takes place prior to secking informed consent and consequently pre-randomization designs are controwersial among ethicists, health lawyers, methodologists, and clinicians. The reticence to use these designs is undeserved, provided that the proper requirements and indications will be applied.
\end{abstract}

Objective Guidelines for the optimal application of pre-randomization designs are presented. For this purpose, designs in which pre-randomization is used are outlined, methodological considerations to choose between conventional designs or pre-randomization designs are described, and relevant ethical and judicial aspects are discussed.

Conclusion In specific situations, based on well-defined indications and recuirements, pre-randomization designs have an essential contribution to achieve evidence-based medicine. Banning pre-randomization a priori implies that effectiveness of many public health and medical interventions cannot be studied. Therefore, every design should be based on a balance between maximizing the potential for patient autonomy and minimizing the bias caused by contamination. Although general rules are important, an independent group of experts, like members of research ethics committees should decide whether this balance is acceptable. 


\section{Introduction}

Nowadays, evidence-based medicine is widely propagated as the reference for good clinical practice. 'This development involves a critical appraisal of a widening range of public health or clinical interventions. As a consequence, the need for patient related research is growing as is demonstrated by the increasing number of scientific publications. In effectiveness studies the conventional randomized trial will always be the design of first choice. However, the conventional design in which informed consent is obtained in all participants before randomization, is not the panacea for all research questions because information in the reference group about the experimental intervention could lead to serious protocol violations. Therefore, in the last two decades several investigators applied pre-tandomization to counter this contamination problem, questioning the absolutism of the conventional design. ${ }^{1-3}$ The term pre-randomiation implies that the randomization takes place prior to seeking informed consent.

Because of this deviant informed consent procedure the position on prerandomization of medical ethicists, health lawyers, methodologists, and clinicians remains to be controversial and varies from total unacceptability to acceptance under specific conditions. ${ }^{4-11}$ Unsurprisingly, there is controversy both between and within Research Ethics Committees (REC) about when, or even whether, these designs are admissible. ${ }^{12}$

Pre-randomization is also applied in apparently less controversial designs in which clusters, like primary cate practices or schools, rather than individuals are randomized and obtaining informed consent from the participants after randomizing the clusters is inevitable. Another situation in which prerandomization is used is in patients who are unable to give informed consent because of a reversible process of disease, like comatose or psychiatric patients who may be able to give consent after a period of convalescence. ${ }^{13},{ }^{1.14}$ Whether the Jatter is justified and in which circumstances a tempontry proxy consent may be used, is a complex issue and the ethical and judicial ramifications are discussed elsewhere. ${ }^{15-18}$ This type of application of the pre-randomization design is beyond the scope of this article.

In this article we derive general guidelines for the optimal application of prerandomization designs. Prior to this, different types of pre-randomization are outlined and an overview of methodological differences between conventional designs and pre-randomization designs is given. In the last section, ethical and judicial considerations concerning trials and informed consent are described.

\section{Pre-randomization designs}

In 1977, Marvin Zelen introduced the randomized single-consent design. ${ }^{10}$, 20 "The key characteristic of this design is that patients are randomized before informed 
consent is sough. "The patients allocated to the experimental treatment group are approachid by the investigator or clinician for informed consent. However, the patents tandomized to the reference group are grven the (best) standard treatinent but are not approached for informed consent. The reason to introduce this design was to avoid problems associated with persuading clinicians to enter patients into clinical cancer trials.20, 21 To overcome ethical concerns, the randomized double consent design as counterpart of Zelen's single-consent design was introduced 2024 In this design, patients are initially offered the treatment to which they were randomized, but informed consent is sought in all patients, irrespective of reatment arm. Usually, a version of the double-consent design is used in which participants in the reference group are informed about their own treatment only and information about the alternative treatment is withheld. Seldom, patients in both treatment groups are fully informed about all major aspects of the trial. ${ }^{12}$

\section{Box 1. Definitions of key concepts used in this paper}

Prawandowization design: study design in which randomization precedes the seeking of informed consent (synonyms: Zelen design, randomized consent design).

Individual fandowigation design study design in which each participant is randomly assigned to a treatment group. The number of tandom assignments equals the number of participants.

Claster randowization design: study design in which the unit of random assignment is a group or a social unit. The number of random assignments is smaller than the number of participants. Examples of clusters are counties, postal code areas, hospitals, or primary care practices.

Single-coment designt: pre-randomization design in which informed consent is sought in the experimental group only, mentioning the use of a reference group (Zelen's proposal of 1977)

Intompletedomble consent derign* pre-tandomization design in which informed consent is sought in the experimental group, mentioning the use of a reference group and in the reference group, not mentioning the use of the experimental group.

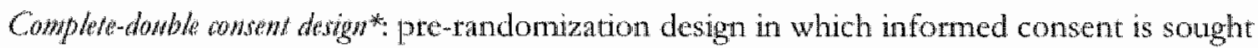
in the index group and in the reference group, mentioning the use of the othet comparison groupn

Simgtededowed consen dorign*: a single consent design in which the participants in the reference group are sought for informed consent at the end of the study (if required for an end measurement with a personal contact).

* Albough it is possible not to mention the use of a reference group in the experimental group or cxen inform the participants in the experimental and reference group only at the end of the srudy. As these vatimts are rarely needed, no distinct definitions ate added.

Chuster randomized trials are becoming increasingly important in health care research, public health research, and general practice research." 25, 26 In cluster randomized trials clusters of people, or intact social units, rather than individuals 
are randomized to an experimental of reference group and outcomes are measured on individuals within these clusters. 27 Cluster randomited trals use pre randomization which means that clusters are andomized before claster nembers are approached for informed consent 28,29 The decision about the participation of a patticular cluster in the trial is sometimes taken before nandonization, by a person who is assumed to represent the participants, ${ }^{30}$ and participants may consent individually to the treatment offered within their cluster after tandomization. 30,31 Box 1 shows the definitions of key concepts that we use in this paper.

\section{Methodological issues for conventional designs and pre- randomization designs}

\section{General issues}

Clinical trials should provide information about the relative magnitude of effect(s) of an expetimental intervention on (disease) outcome as compared to some reference intervention(s) or policies. This information is ultimately obtained by comparing the outcomes of interest between the study gtoups. The difference thus calculated may be explainable by different mechanisms. First, it could be the result of the experimental intervention. Second, it could be the result of bias. Briefly, bias may result from differences in the (joint) distributions of prognostic indicators between the two study groups at baseline, differences in the frequency with which effective co-interventions were applied during the study period, and differences in the way the outcomes were measured. Finally, chance may allways explain the findings, however unlikely given a small p-value or another statistical measure of the precision of the estimate. Thus, the internal validity (scientific quality) of any study is inversely associated with the number of ways the findings can be interpreted. Ideally, a study allows only one interpretation, namely, that the experimental intervention explains the differences found. An approptiate study design is a prerequisite to achieve this goal.

The two most important maneuvers used in clinical trials ate randomization and (different forms of) blinding. Randomization is intended to make the study groups prognostically comparable at baseline. Blinding aims to keep participants, caregivers, and/or those who measure outcomes unaware of treatment allocation. This is important when it can be expected that knowledge about the intervention or even the fact that randomization occurred, may change behavior of participants, caregivers, and/or may influence those who measure outcomes in a way that will disturb the comparability of the study groups.

In general, the most important types of protocol violations that seriously can endanger the internal validity of a study ate dropout and non-compliance. We define a dropout as a randomized participant whose outcome measurement is not obtained and, as a consequence, cannot be included in the analysis of outcome, or 
a (few) well chosen) value(s) for this measurement is substituted or imputed. When the arerage outcomes of the dropouts differ between the treatment groups the validity of the results is violated. We define a non-complier as a participant who deviates from the protocol with respect to his/her allocated treatment by refusing the allocated treatment altogether, taking the allocated treatment in a way that negavively affects its effect, or by taking alternative and effective treatment. Non-complance influences the contrast between the study groups, and, even in an intention-tomtreat analysis, causes biased results. Most of the time, non-compliance dilutes the intended contrast and will thus cause underestimation of the true treatment effect(s). Furthermore, as non-compliance can, in principle, be measured and sometimes adjusted for, it generally seems a somewhat less serious threat to internal validity than dropout.

\section{Protocol violations in conventional designs and pre-tandomization designs}

Especially participants who are dissatisfied with their treatment allocation may decide to withdraw or stop their full participation (dropout) thereby possibly causing irteparable selection bias. Dissatisfaction may also lead to non-compliance because participants seek other treatments, which, if effective, may cause betweengroup incomparability at the level of co-interventions, which is difficult to deal with data-analytically. Finally, dissatisfaction may have a negative attitude to the outcome measurement(s) and may lead to incomparability that cannot be adjusted for. One solution for this problem is blinding and the need for blinding is determined by the extent to which the aforementioned mechanisms render the study's findings open to more than one interpretation.

One way of blinding participants is the use of a convincing placebo or sham procedure. However, with some interventions the use of a placebo or sham procedures is infeasible since they would have to mimic peculiar (physiological) effects that are inseparable from the effect of the experimental treatment. Think, for example, of a trial on heroin provision, an attractive intervention, in which using a placebo is impossible. In this case premandomization could be an alternative to maintain the internal validity of such a study.

The influence of the use of pre-randomization on a study's internal validity as compared with the conventional design is elaborated by means of Figure 1 . This Titgure shows the occurrence of the different types of protocol violations and non participation for the conventional design and the single-consent design (Zelen's original proposal). Non participation in the trial and/or protocol violations may occur due tro Gactots related to the experimental treatment, the reference treatment, or due to factors unrelated to the treatments. These are factors related to the burden of the effect measurements, or to the randomization procedure, or factors based on tandom processes like emigration et cetera. These factors are explained in the legend to Figure 1. 


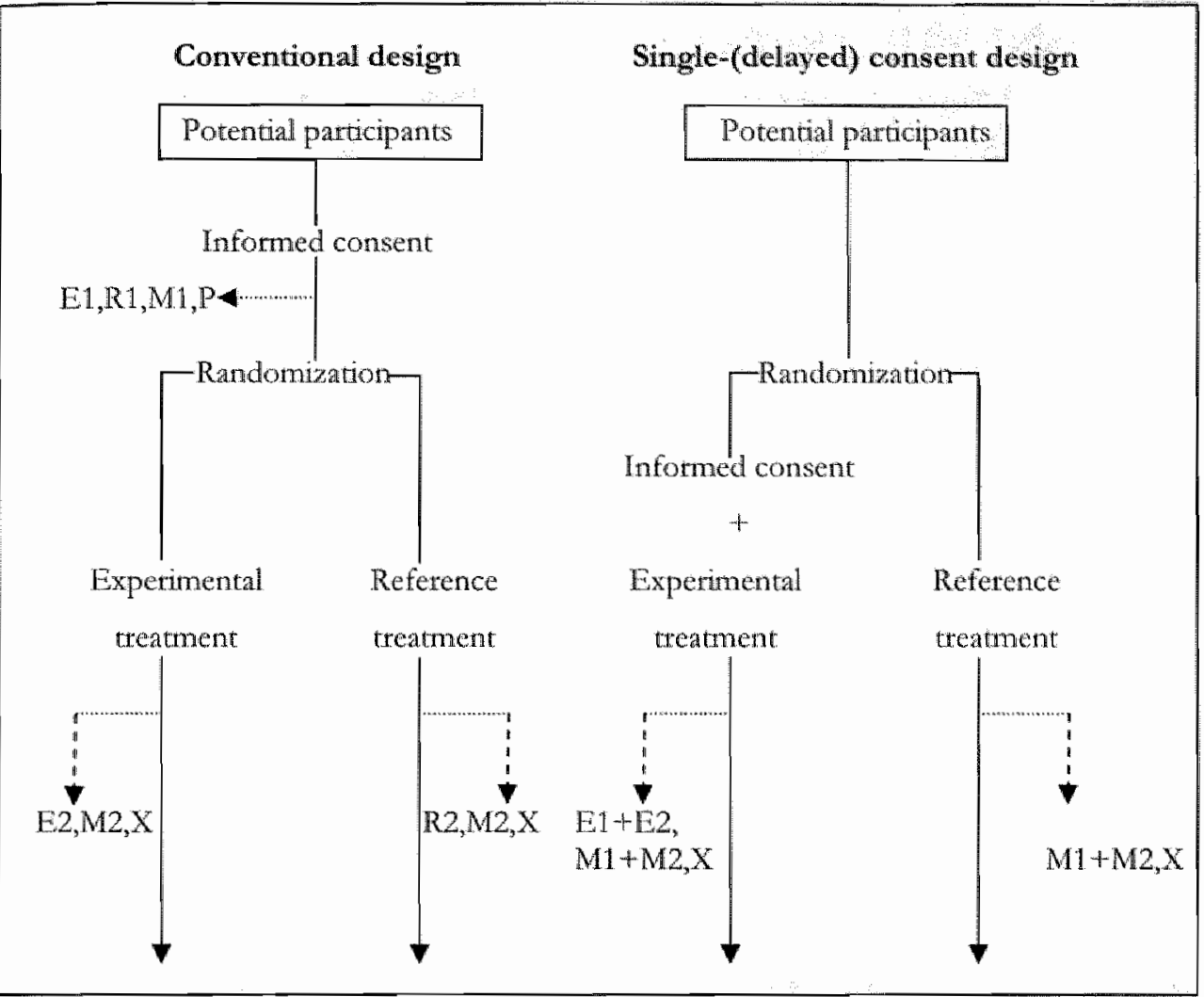

Figure 1. Factors causing protocol violations and non-participation in a conventional design (left panel) and in a single-(delayed) consent design (right panel) as defined in Box 1.

\section{Legend to Figure 1.}

Factors causing non-participation and/or protocol violations in a trial

E: Factors related to the experimental treatment include inconveniences due to complications, side effects and extra efforts associated with the experimental treatment. The (eligible) participant may anticipate (any of) these inconveniences (dangers) and refuse co-operation at the patient recruitment procedure (E1) or experience (any of these) effects during the trial and decide to become a non-complier and/or drop out completely (E2).

In case of an attractive experimental treatment $\mathrm{E} 1$ and $\mathrm{E} 2$ will be negligible small in a single-consent design and will not bias the tesults.

R: Factors related to the reference treatment include the expected attractiveness of the alternative, that is, the experimental treatment. The eligible participant might refuse cooperation at the parient recruitment procedure (R1) or, in the case of a conventional design, take a chance on a desirable outcome of the randomization procedure but withdraw if $s$ /he is allocated to the reference group (R2).

In case of an attractive experimental treatment, the protocol violations of group $\mathrm{R} 2$ could considetably bias the results of a conventional design. 
M: Factors telated to the burden of the effect measurements during and at the end of the study. This also can be anticipated at the including procedure (M1) or during the trial (M2). These factors will be balanced berween the treament groups.

P. Factors related to the randomization procedure, for example because of a general awersion to lotterics.

X: Random processes causing withdrawal such as emigtation, traffic accidents, family atfairs et cetera.

These factors will be balanced between the treatment groups.

In the pre-randomization design (Figure 1, right panel), all eligible participants are randomized and thus included in the study. In the conventional design (Figure 1, left panel), potential participants get the opportunity to refuse participation prior to randomization. In contrast to the protocol violations related to the treatments, those protocol violations caused by the burden of the effect measurements or random processes will be balanced berween the treatment groups in both designs. In the case of an attractive experimental treatment, contamination in the reference group could lead to search for an alternative treatment, and/or refusing coopetarion, or withdrawal and in this way considerably bias the tesults of a conventional design. In the pre-randomization design we expect that this contamination is avoided and consequently protocol violations will be negligible. In this design, the effect measurement in the reference treatment group could lead to contamination if an individual approach is necessary. Individual measurements at the start or during the trial would reveal the purpose of the study and cause protocol violations. One way to overcome this problem is to obscure the purpose of the trial but that would be ethically and judicially questionable. If no individual baseline measurements are necessary, another approach would be to find a way to persuade the participant to comply with the outcome measurements at the end of the study. In this case the informed consent procedure in the reference group is delayed (single-delayed consent design, see Box 1).

Thus, methodologically the pre-randomization design seems preferable when an attractive experimental treatment is involved, the reference is the standard treatment, the use of a sham procedure or placebo cannot be used, and possible contamination caused by outcome measurements may be prevented.

This also applies for trials in which the information given during the informed consent procedure could be (a part of) the intervention, like for instance a health promotion trial. The knowledge or awareness of this intervention would also have the (intended) trial effect in the reference group and could influence the behavior of the participants in the reference group. For instance, investigating the effect of education about healthy food, is only possible if the reference group is not alerted. In a screening trial on breast self examination to early detect breast cancer, individual randomization and informed consent in all participants would alert the 
participants in the refetence group. As a consequence a part of these participants would perform breast self-examination or search for alternatives like a referral to the radiologist and in this way affect the contrast between the study groups.

Knowledge about the experimental intervention could have (partly) the same effect as intended in the experimental group. If the intervention consists of giving information with a negligible burden for the participants, for instance additional information about dietetics in a standard health education program for students, a design in which incomplete informed consent (see Box 1), or even no informed consent is obtained in all participants might be methodologically attractive.

\section{Guidelines for the use of pre-randomization designs}

The diagtams shown in Figure 2 can support making an optimal choice between the different types of pre-randomization designs. A prime issue in selecting a study design is the level of the intervention. If each participant is randomly assigned to a treatment group, an individual randomization scheme may be used. If the intervention is only feasible at an aggregated or clustered level, such as school classes, hospitals or primary care practices, the unit of random assignment is a cluster. Depending on the level of the intervention, a diagram offers guidance.

Furthermore, if in Figure 2 serious protocol violations are anticipated, but cannot be avoided, consider not conducting the study.

\section{Ethical and judicial considerations}

In a clinical trial there is a balance between individual and collective ethics, ${ }^{32,33}$ 'Individual ethics' implies that each patient should teceive that treatment which is thought to be most beneficial for his condition. This is the clear aim of good clinical practice in which the patient and his clinician decide together on the best course of action. Usually, the clinician ptoposes the therapy on the basis of his knowledge and experience, with appropriate acknowledgement of the patient's wishes.

'Collective ethics' is concerned with societal benefit. By achieving medical progress as efficiently as possible, future patients may benefit from superior therapy. Exclusive adherence to collective ethics is unacceptable as it implies that clinical trials can be conducted whille ignoring the needs of the participants and solely complying with scientific and statistical principles. Some authors instinctively pay exclusive attention to individual ethics. Adoption of this attitude implied that properly designed clinical trials could not be performed. We would thereby lose our best tool to tell effective and ineffective medical treatment apart. In randomized double-blind trials patients should be unaware of the treatment allocation. A commitment to individual ethics only, would appear contradictory to any use of randomization, blinding and placebos. 


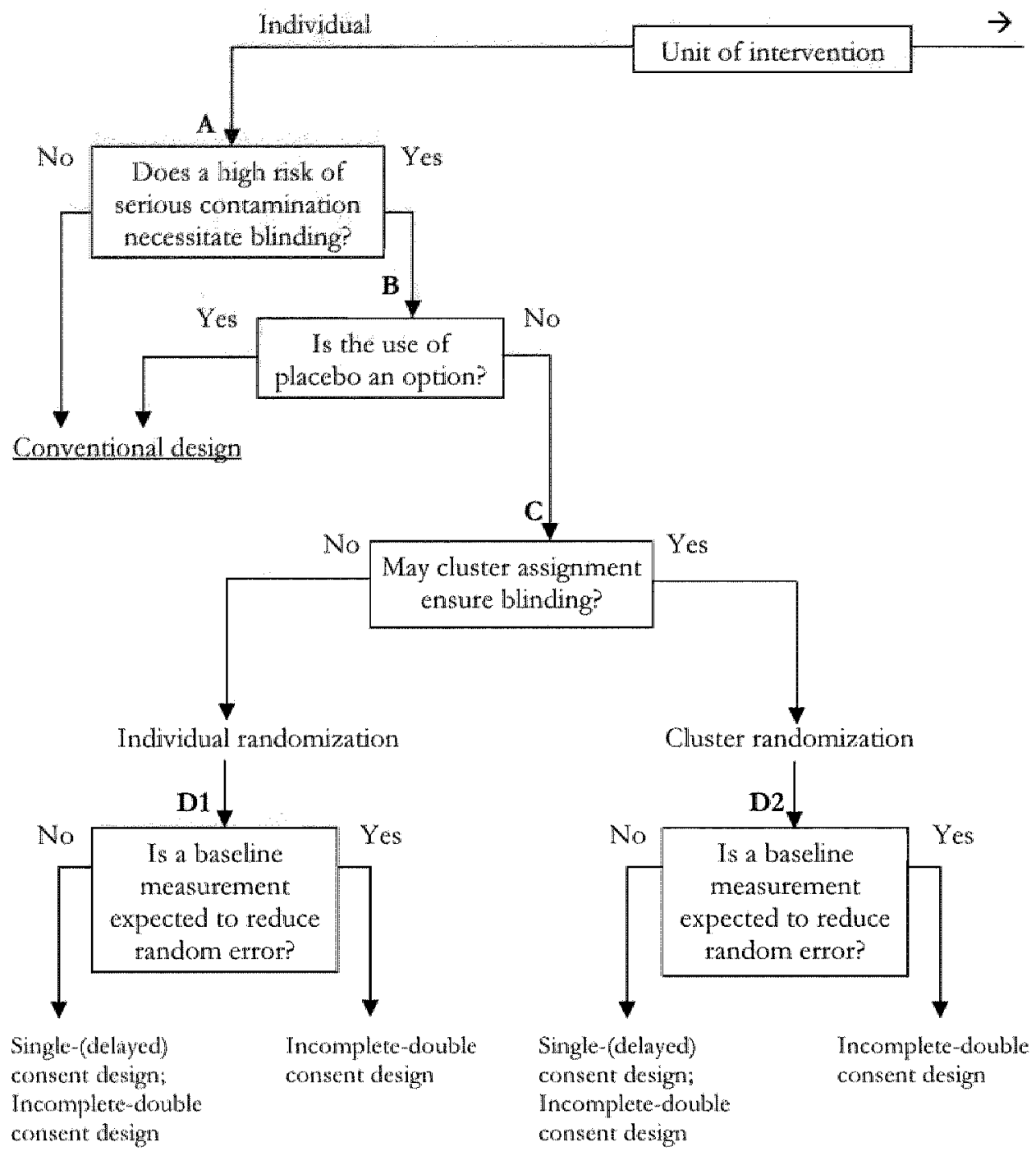




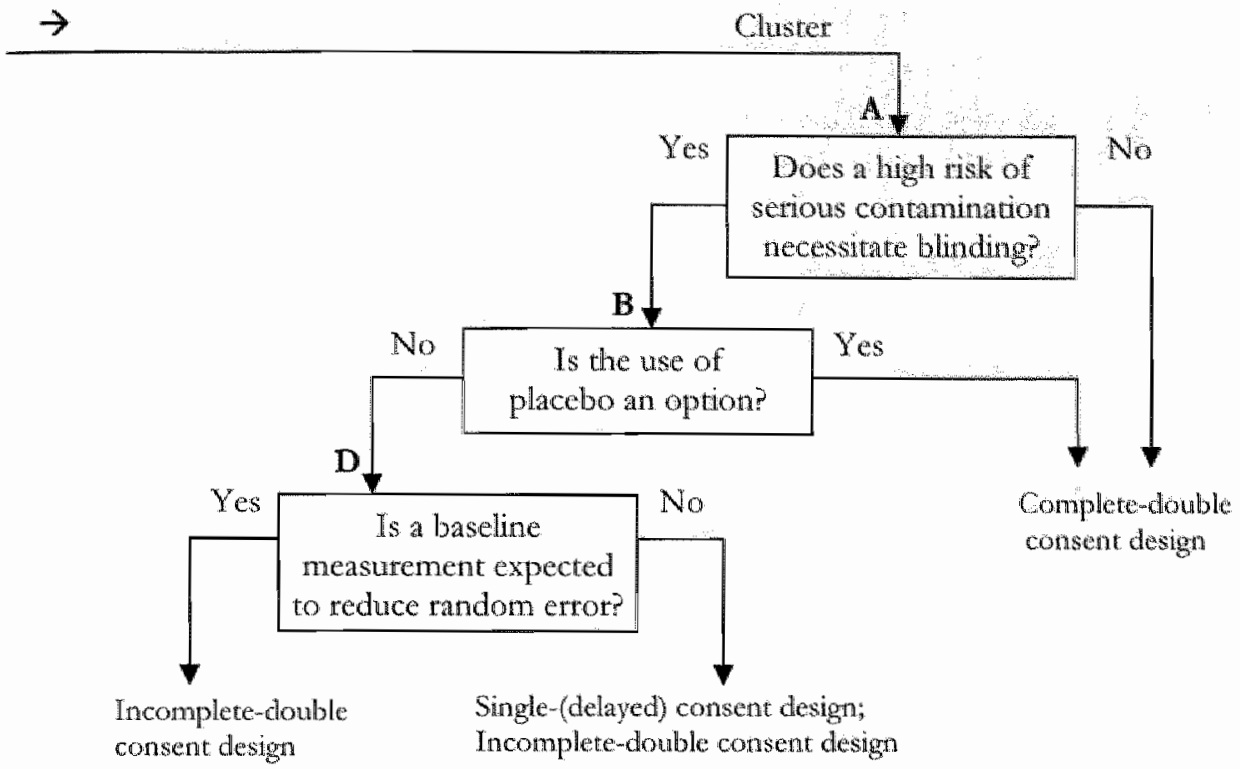

Figure 2. Diagrams for the use of pre-tandomization designs.

\section{Legend to Figure 2.}

Node A: If serious protocol violations (see Figure 1) are anticipated in the reference group that may be avoided by blinding the participants in this group, go to $\mathbf{B}$. If blinding is not necessary, use the conventional design.

Node B: Can using a placebo in the reference group prevent protocol violations? If not, a pre-randomization design might be a solution for the blinding problem, go to $\mathbf{C}$.

Orherwise use a conventional placebo controlled design.

Node C: Is a cluster assignment a solution for blinding the participants for the alternative treatment? If not, go to D1. If so, go to $\mathbf{D} 2$.

Node D \& D1 \& D2: If a baseline measurement is required and this information cannot. be obtained by a register or data collected routinely, a personal contact with the participant is necessary. In this case the participants in the reference group have to be informed about: the reason for this measurement without alerting these participants with the knowledge about the experimental treatment: i.e. the incomplete double consent design.

The most widely accepted ethical code for medical research is the Declaration of Helsinki. ${ }^{34}$ In addition, in most countries legislation and/or guidelines are promulgated for the application of informed consent procedures in clinical trials. ${ }^{35-}$ ${ }^{37}$ Many lawyers interpret these guidelines as prohibiting research with individuals if informed consent or consent by proxy is not or not fully sought. Others accept not obtaining informed consent in very specific cases as conceivable or at least as not explicitly excluded by law. 
To protect the individual autonomy an eligible person at rrial inclusion should be enalbled to weigh the likely pros and cons of participation and infringement of his private interest against potential benefits for society. However, the goal of informed consent is often imperfectly realized as sometimes complex information must be given about the treatment options. ${ }^{3 *}, 39$ The informed consent procedure has been the subject of ethical and judicial debates. ${ }^{8}, 40-44$ Special attention is given to circumstances in which informed consent is not obtained. ${ }^{15}$ - First, the eligible person may be unable to give consent in case of minority or in case of specific neurological or psychiatric disorders. Another circumstance occurs in cluster randomization trials in which an informed consent procedure at the level of those who undergo the intervention would require a great deal of disproportional, logistical effort and the intervention does not infringe on someone's private interest. $A$ third condition appears when a regular informed consent procedure would endanger the trial's internal validity (as described in the previous section). In pre-randomization designs one may distinguish between different informed consent procedures ranging from no information to full information (see Box 1). Which of these options should be considered in a study protocol should be determined by weighing methodological requirements against the ethical principle of an individual's autonomy and being completely informed in advance.

If a baseline measurement is not required, also a single consent design, in which the participants in the reference group are not informed, could be an option. If a measurement with personal contact at the end of the study is necessary, inform the participants in the reference group and ask their consent at the end of the study: i.e. the single-delayed consent design. If the experimental intervention involves only a minimal burden for the participants a design without informed consent could be an option.

\section{Conclusion}

In general, the usefulness of pre-tandomization in study designs is undervalued. However, based on well-defined indications and requirements, pre-randomization designs may be essential to evidence-based medicine. Banning pre-randomization a priori implies that information about the effectiveness of important public health and medical interventions will not be forthcoming. A number of effective interventions will not be introduced because of the lack of evidence. Furthermore, the use of established, (possibly) ineffective interventions will be continued without evidence of net benefit.

In our opinion, every design should be based on a balance between maximizing the potential for patient autonomy and minimizing the bias caused by contamination. The combination of varieties of research questions and contexts, in which a study has to be conducted, necessitates a tailor-made design. In every 
design proposal the investigator searches for a balance between optimal methodology, using for instance our guidelines as proposed in this article, and his/her personal assessment of ethical implications. As this balance cannot be fully reached by formulating general nules, the final decision whether this balance is acceptable, should be made by independent group of experts, like members of a research ethics committee. 


\section{References}

1. Knotmerus $\mathrm{J} A$. Health care research in ambulatory care. In: Ethick en recht in de gezondheidszorg (Dutch language). Deventer: Kluwer; 1997. p. IV 151-94.

2. Schellings $R$, Kessels $A G$, ter Riet $G$, Sturmans $F$. The Zelen design may be the best choice for a hetoin-provision experiment / Clin Epidemiol 1999, 52: 503-07.

3. Gallo $\mathrm{C}$, Perrone $\mathrm{F}$, De Placido $\mathrm{S}$, Gusti $\mathrm{C}$. Informed vetsus tandomised consent to dinical trials. Lancet $1995 ; 346: 1060-4$.

4. Eillenbarg SS. Randomization designs in comparative clinical trials. New Engl J Med 1984; $310: 1404-08$.

5. Ellenberg $\$$ S. Informed consent protection or obstacle? Some emerging issues. Controlled Clin Trials 1997; $18: 62836$.

6. Beauchamp TL, Cook RR, Faverweather WE, Raabe GK, Thar WE, Cowles SR, Spivey GH. Ethical guidelines for epidemiologists. J Clin Epidemiol 1991; 44: 151S-69S.

7. Medical Research Council Cancer Thetapy Commitee. Investigations on human subjects: ethical considerations in the study of cancer therapy. CTG $86 / 24$, February 1987.

8. Gezondheidsrand. Informed consent en prerandomisatie. Den Haag: Gezondheidsraad, 1999.

9. Homer CS. Using the Zelen design in randomized controlled trials: debates and controwersies. I Adv Nurs 2002; 38:200-7.

10. Palmer CR. Ethics, data-dependent designs, and the strategy of clinical trials: time to start learning-as-we-go? Stat Methods Med Res 2002; 11: $381-402$.

11. Marquis D. An argument that all prerandomized clinical trals are unethical. J Med Philos 1986;11:367 83.

12. Schellings $R$, Kessels AG, Riet ter $G$, Kleipnen J, Leffers P, Knotmerus JA, Sturmans $F$. Members of research ethics committees accepted a modification of the randomized consent design. J Clin Epidemiol 2005; 58: 589-94.

13. Luce JM. Research ethics and consent in the intensive care unit. Curr Opin Crit Care 2003; 9: 540-4.

14. Collins PJ, Jarkin EP, Shubsachs AP. Lithium carbonate in chronic schizophrenia: a brief trial of lithum carbonate added to neuroleptics for treatment of resistant schizophtenic patients. Acta Psychiatr Scand 1991; 84: 150-4.

15. Rees 1 , Hardy $J$. Nowel consent process for tesearch in dying patients unable to give consent. BM] 2003; 327: 198-1.

16. Silverman $\mathrm{H}_{\mathrm{J}}$, Luce JM, Schwartz J. Protecting subjects with decisional impairment in research: the need for a multifaceted approarch. Am I Crit Care Med 2004; 169: 10-4.

17. Dimond B. Consent and the mentally disordeted detained patient. Br J Nurs 2003; 12 ; $1331-4$.

18. Dijk Y wan, Voont PH wan der, Kuiper MA, Kesecioglu J. Research on subjects incapable of giving informed consent: the situation in Dutch intensive cate departments. Intensive Care Med 2003; 29: 2100-1.

19. 2elen M. Statistical options in dinical trials. Semin Oncol 1977; 4: 441-46.

20. Zelen M. A new design for andomized clinical trials. N Engl J Med 1979; 300: 1242-5.

21. Zülen M. Strategy and alternate randomized designs in cancer clinical trials. Cancer Treat Rep 1982; 66: 1095-1100.

22. Zelen M. Innovations in the design of clinical trials in breast cancer. Breast Cancer Res Treat $1983 ; 3: 137-42$

23. Zeten M. Randomized consent designs for clinical trials: an update. Stat Med 1990; 9 : 645-6. 
24. Alman DG, Whitehead ], Parmar MK, Stenning SP, Fiyers PM, Machin D. Randomised consent designs in cancer clinical trials. Eur J Cancer 1995; $31 \mathrm{~A}: 1934,4$.

25. Campbell MJ. Extending CONSORT to include cluster trials. BMY 2004; $328: 6545$.

26. Campbell MK, Grimshaw JM, Elbourne DR. Intracluster correlation coefticients in cluster randomized crals: empirical insight into thow should they be reported. BMC Med Res Methodol 2004; $4: 9$.

27. Medical research Council. MRC guidelines for good clinical practice in clinical trials. London: Medical Research Council, 1998.

28. Torgerson DJ. Contamination in trials: is cluster randomisation the answer BMJ 2001 ; 322: $355-7$.

29. Piaggio G, Carroli G, Villar J, Pinol A, Bakketeig L, et al. Methodological considerations on the design and analysis of an equivalance stratistied cluster randomization trial. Stat Med 2001; 20:401-16.

30. Edwards SJL, Braunholtz DA, Lilford RJ, Stevens AJ. Ethical issues in the design and conduct of cluster randomised controlled trials. BMJ 1999; 318: $1407-9$.

31. Klar $\mathrm{N}$, Donner $\mathrm{A}$. Current atnd future challenges in the design and analysis of cluster randomization trials. Stat Med 2001; 20:3729-40.

32. Palmer CR, Rosenberger WF. Ethics and practice: altemative designs for phase III randomized clinical trials. Controlled Clin trials 1999; 20: 172-86.

33. Pocock SJ. Clinical trials. Chichester: Willey; 1993.

34. World Medical Association Declatation of Helsinki. Ethical principles for nedical research involving human subjects. JAMA 2000; 284: 3043-45.

35. Ministry of $\mathbb{H e a l t h}$, Welfare and Sports. WMO: The Medical Research Involving Human Subjects Act. The Hague: Ministry of Health, Weltare and Sports, 1998.

36. Medical Research Council Cancer Therapy Committee. Investigations on human subjects: ethical considerations in the study of cancer therapy. CTG 86/24, February 1987.

37. Final rule-final regulations amending basic HHS policy for the protection of human research subjects (45 CFR Part 46). Federal Register. January 26, 1981; 46:8366-91.

38. Laforet $G G$. The fiction of informed consent. JAMA 1976; 235; 1579.-85.

39. Katz J. Informed consent. Univ Pittsburgh Law Rev 1977; 39: 137-74.

40. Smith R. Informed consent: the intricacies. BMJ 1997; 31 4t: 1059-60.

41. Informed consent in medical reseatch [letters]. BM] 1997; 314: 14707-83.

42. Informed consent [letters]. BMI 1997; 315:247-54.

43. Torgerson DJ, Roland M. What is Zelen's design BMJ 1998; $316: 606$.

44. Smith R. Informed consent: edging forward (and backwatds). BMI 1998; 316: 949-51. 


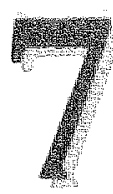

General discussion 



\section{Introduction}

This chapter discusses the findings of the study and focuses on general aspects. Specific points of interest have been dealt with in the discussion sections of the respective chapters.

\section{Implications for research: methodological guidelines for ethical research}

Clearly, evidence-based medicine is the reference for good clinical practice, health care, and public health. Parient related and public health effectiveness studies serve this purpose and by far the majority of these studies use a conventional randomized study design. However, the conventional design is not the panacea for all research questions and investigators become aware that pre-randomization. could be an alternative under strict conditions.

In general, pre-randomization is justified if the following conditions are met? the study has a clinically relevant objective; it is likely that the study will lead to important new insights; a valid evaluation of the effects is impossible using a conventional randomized design; the informed consent procedure bears no potential harm to participants; at least usual care is offered to participants in the reference group; and the approval of an independent research ethics commitree.

The findings of our study show that, methodologically, pre-randomization is useful when an attractive or preferable experimental treatment is involved, the reference is the standard treatment, the use of a sham procedure or placebo cannot be used, and using pre-randomization can minimize bias caused by contamination. Besides, in case of a single-consent design the choice depends on weighing the loss of validity due to selective dropout and non-compliance in the reference group against the potential loss of precision due to not performing a baseline measurement.

In particular, the starting point for conducting a trial is to obtain an optimal comparison of the outcomes of interest between the study groups. For this purpose, regardless of the study objective, the investigator must assess the likelihood that contamination will spoil the experimental contrast. Randomization and different forms of blinding are important in clinical trials to achieve internal validity, but these maneuvers must not be used as "standard tituals', 2, 3 Methodological guidelines, as presented in chapter 6, may lead the investigator to an appropriate study design, in addition to which the protection of the autonomy of participants and causing no harm or burden to them is the ethical guide.

An interesting viewpoint on blinding study participants is described by Rothman and Michels., ${ }^{4}$ In the spirit of the most recent (Fifth) Declaration of Helsinki they contended that the use of placebo controls in clinical trials is generally unacceptable (this is unethical) when standard therapies or interventions 
are avallable for a particular patient population. Against the background of this statement our guidelines, described in chapter 6 , may lead to a suitable study design. If there is no high risk of serious contamination that necessitate blinding a conventional design is the best choice. Othervise a pre-randomization design might be an alternatuve for ensuring blinding the study participants for the alternatrve treatment.

Anyhow, based on well-defined indications and requirements, prerandomization destgrns have an essential contribution to evidence based medicine. Banning premandomization designs implies that knowledge about the effectiveness of many public health, medical, and clinical interventions will not be available. A number of potentially effective interventions will not be introduced because of the lack of eviclence. Furthermote, the use of established, (possibly) ineffective intervention, like alternative medicine or quack remedies, will be continued without evidence that the benefit prevails the harm.

The critical contribution of an independent group of experts, like members of research ethics commitrees, in the approval of study proposals is essential to protect health care and public health now and in the future.

\section{Research ethics committees as ethical and methodological watchdogs}

Research ethics committees or another independent groups of experts, are impottant instruments to preclude arbitraniness and to prevent too much personal influence of investigators, ethicists, lawyers, methodologists, and physicians on the acceptance of a study proposal. Therefore, independence of review is an important element of good research governance.

Our survey on the acceptability of randomized consent designs (chapter 5) demonstrates that members of research ethics committees make a well-balanced judgement about the acceptance and rejection of randomized consent designs. Prerandomization in indidually andomized trials is acceptable for many members of research ethics commitrees if the study design is presented in an ethically wellconsidered way. Although the differences between members and between committees are substantial, some variation in the judgement of study proposals may be acceptable. However, inappropriately high variability can impede the progress of research." On an international level this problem is obvious since countries clearly differ in their requirements for approval by a research ethics commitree for an identical study. ${ }^{7}$

In spite of the secrecy that still surtounds research ethics committees and their decision making, 8 it seems that members of research ethics committees do not shut the doot for a discussion about the usefulness of controversial designs like pre-andomization designs. Guiding for such a discussion must be the balance between methodology and ethics, on which every design must be based. 


\section{The ethics of informed consent: the right information at the right moment}

The most influential source of guidance in the design and implementation of ethical research in human beings is the Declaration of Helsinki. "This declaration and derived national, and local laws, and guidelines, must not be conceived too much as a straitjacket of all sorts of regulations. Regulations must give trialists the opportunity to balance methodology and ethics in the design and implementation of a trial.

In addition to worthwhile study objective and good methodology informed consent is fundamental to the ethics of conducting trials and it is viewed as the key to respecting study participants' autonomy. Respecting this means that informed consent should not be an end in itself, but a means to protect the individual and to guarantee the right of autonomy (chapter 2). On the other hand, physicians who conduct research are using the patients as a means to an end. Their goal is no longer solely the wellbeing of the patients. They are also, perhaps primarily, committed to creation of generalizable knowledge. If so, the goal of ethical guidelines might also be the acknowledgement of this conflicting interest. ${ }^{9}$ Besides, the ultimate importance of informed consent is violated by the view that complete, specific consent in full detail is an illusion, ${ }^{10}$ the question to what extent informed consent is required and in what form remains open, ${ }_{y}{ }^{11}$ and both the timing of the informed consent procedure and the content of the informed consent are still part of an (ongoing) discussion. 12.24

In pre-randomization designs, cluster or individually randomized, there is a broad variation of informed consent procedures, ranging from full consent in all participants in all study groups to no consent at all.

The informed consent procedure in pre-randomization designs differs from the procedure in a conventional design in an essential way. Pre-randomization implies that participants are not asked to consent to (their) randomization, but only to undergoing treatment(s) and effect measurement(s). This is one of the major objections against pre-randomization in an individually randomized trial because participants in the reference group are unawate of their participation in the trial, participation in the reference group would not be voluntary, and participants in the reference group cannot willfully withdraw from the trial. However, a fundamental question is whether these participants, who receive standard treatment, are subjects whose autonomy is violated only because they are randomized. We take the position that autonomy is respected and no burden or harm is caused as long as a personal approach for measurements is not necessary. There is no question that informed consent is required by such a personal approach for baseline and effect measurements (if not yet routinely available), as we demonstrate in chapter 4 by means of a fictitious heroin-provision experiment. 


\section{Pre-randomization designs were rarely used, sometimes underused, and often badly designed}

In our systematic literature search (chapter 3) we define pre-randomization designs with individual randomization as randomized consent designs. Since Marvin Zelen introduced his single-consent design in 1977, the randomized consent design has been rarely used in published research. We detected 50 studies until 2002. Besides, for the majority of the studies the use of a randomized consent design was unjustifted because the most important protocol violations, namely, noncompliance and dropout, seriously endanger the internal validity of the studies. In these studies the use of a randomized consent design was not restricted to avoid contamination, but, among other things, also to avoid problems expected with the randomization procedure. This seems not to be a valid reason to preserve the internal validity of a study (chapter 6).

In a review of recent cluster randomized trials published in three general and leading medical journals from 1997 to 2002, the authors identified 42 potentially eligible trials. ${ }^{25}$ They reviewed 36 trials and concluded that cluster trials are vulnerable to the risk of bias at cluster level, and at individual level. According to the authors, some cluster randomized trials may not have taken adequate precautions to guard against threats to the internal validity of their design. Like others, 26,27 they argue for amending the CONSORT statement to allow for the special methodological circumstances of cluster trials. The statement includes a checklist of items that should be included in the trial report and is already available for individually randomized trials.

It is remarkable that, in the literature, cluster randomized trials raise less ethical objections than individual randomized trials that use pre-randomization. 1,28 Both designs use pre-randomization, but cluster randomized trials are discussed methodologically ${ }^{2032}$ rather than ethically. ${ }^{33}$ Perhaps this difference could be explained by the nature of the intervention. Trials randomizing clusters have become particularly widespread in the evaluation of non-therapeutic interventions, including lifestyle modifications, educational programs, and innovations in the provision of health care. When the intervention is of minimal or no impact on the indivicluals concerned, the investigator and the research ethics committee may handle less stringent conditions on ethical requirements. On the other hand, as shown in chapter 3, the subject of randomized consent designs, in which individuals are randomized, changed over years from medical to behavioral interventions. In spite of this, these designs are still being ethically criticized, even if they are well designed methodologically.

Because of the reticent use of pre-randomization in study designs, it might be possible that pre-randomization designs are currently underused or have been underutilized. Interesting in this matter is the statement that the Zelen design or single-consent design may be the best choice for a heroin provision experiment, as 
described in chapter 4. In 1998 a conventional randomized controlled thial started in the Netherlands investigating the effectiveness of prescription of heroin added to regular methadone treatment. ${ }^{3 *}, 35$ Using a randomized consent design could have prevented some methodological shortcomings, like the parly improper inclusion of eligible addicts, and non-balanced non-compliance and dropout between the experimental and the reference groups. Unfortunately, it is unlikely that the opportunity to prove this statement will occust.

\section{Pre-randomization needs a reappraisal}

In general, a renewed interest for pre-randomization in study designs could contribute to a better quality of scientific research, and evidence-based medicine. For this purpose, ethical, methodological, and judicial requirements must be discussed without prejudices, but based on walid arguments. This thesis may contribute to this discussion. 


\section{References}

1. Knottneru JA. Gezondheidszorgonderzoek in extramurale setungs. In: Ethiek en recht in de gezondheidszorg. Deventer: Kluwer; 1997. p. IV 151-94.

2. Altman $D G$, Bland JM. Treatment allocation in controlled trials: why randomise? BM] 1999: $318: 1209$.

3. Knipschild PG. Dwalngen in de methodologie. XXII. De noodzaak van tandomiseren en blinderen in therapeutisch onderzok. Ned Tijdschr Geneeskd 2000; 144: 1826-8.

4. Rothman $\mathrm{KJ}$, Michels $\mathrm{KB}$. The continuing unethical use of placebo controls. N Engl J Med 1994, 331: 394 8.

5. Michels KB, Rothman KJ. Update on unethical wse of placebos in randomised trials. Bioethics 2003; 17: 188-204.

6. Wagner TH. Improving the decision processes of institutional review boards. JAMA 2004: $291: 1698$.

7. Hcarnshaw $H$. Compatison of requirements of research ethics committees in 11 European countries for a non-invasive interventional study. BMJ 2004; 328: 140-1.

8. Ashcroft R, Pfeffer $\mathrm{N}$. Ethics behind closed doors: do research ethics committees need secrecy? BMJ 2001; 322: 1294-6.

9. Jantos JD. The "inclusion benefit" in clinical trials. J Pediatr 199; 134: 130-1.

10. O'Neill O. Some limits of informed consent. I Med Ethics 2003; 29:4-7.

11. Sreenivasan $G$. Does informed consent to research require comprehension Lancet $2003 ; 362: 2016-8$.

12. "Truog RD, Robinson W, Randolph A, Morris A. Is informed consent always necessary" for randomized controlled trials? N Engl J Med 1999; 340:804-7.

13. Craen AJM de. 'Informed consent' en prerandomisatic. Ned Tijdschr Geneeskd 2000; 144: $1301-2$.

14. Gevers JKM. Medisch wetenschappelijk ondernoek met mensen. Tijdschrift voor gezondheidsreche $2001 ;$ (1): $22-8$.

15. Willison DJ, Keshavjee K, Nair K, Goldsmith C, Holbrook AM. Patient consent preference for research uses of information in electronic medical records: interview and survey data. BMI) 2003; 326:373-7.

16. Boter H, Delden JJM van, Haan RJ de, Rinkel GJE. Patient's evaluation of informed consert to postponed information; cohort study. BMJ 2004; 32\%. 86-7.

17. Rees $\mathrm{E}$, Hardy J. Novel consent process for research in dying patients unable to give consent. BM] 2003; 327: 198-201.

18. Boter H, Delden JJM van, Haan RJ de, Rinkel GJE Modified informed consent procedure: consent to postponed information. BMJ 2003; 327: 284-6.

19. Shannon C. Berter communication is key to rectuiting patients to trials. BMJ 2003; 327: 1368.

20. Kodish E Eder M, Noll RB, Ruccione K, I ange $\$$, Angiolillo A, et al. Communication of randomization in childhood leukemia trials. JAMA 2004; $291: 470-5$.

21. Whiney SN, McGuire AL, MCCullough LB. A typology of shared decision making, intomed consent, and simple consent. Ann Intetn Med 2003; 140 : 54-9.

22. Tu JV, Willison DJ, Silver PL, Fang J, Richards JA, Laupacis A, et al. Impracticability of informed consent in the registry of the Canadian Stroke Network. N Engl I Med 2004; $350 \times 1414-21$.

23. Lerner BH. Sins of omission-cancer research without informed consent. N Engl J Med $2004 ; 351: 628-30$. 
24. Berghmans RL, Widdershoven GA Ethical perspectioeson decusion-making eapacity and consent for trement and research. Med Law $2003,22,391,400$.

25. Puffer $\mathrm{S}$, Torgerson DJ, Watson J. Evidence for risk of bias in chuster ramdonised ruals: review of recent trials published in three general medical hournals. BMJ 2003, 327: 785. 9.

26. Campbell MK. Grimshaw JM. Cluster randomsed trials: time for improvement. BM] $1998 ; 317: 1171-2$

27. Campbell MK, Elbourne DR, Alman DG. CONSORT" statement: extension no cluster randomized trials. BM] 2004; $328: 702-8$.

28. Edwards SJL, Lilford RJ, Braunholtz DA, Jackson JC, Hewison J, Thornton J. Ethical issues in the design and conduct of randomised controlled mials "Health Technol Assessment 1998; 2 (15).

29. Paggio G, Carroli G, Villar J, Pinol A, Bakketeig L, et al. Methodological considerations on the design and analysis of an equivalence stratified cluster randomization trial. Stat Med 2001; 20: $401-16$.

30. Klar $N$, Donner $A$. Current and future challenges in the design and analysis of cluster randomization trials. Stat Med 2001; 20: 3729-40.

31. Torgerson DJ. Contamination in trials: is cluster randomisation the answer? BMJ 2001 ; $322: 3557$.

32. Fayers PM, Jordhøy MS, Kaasa S. Cluster-randomized trials. Palliarve Medicine 2002 : 16: $69-70$.

33. Edwards S]L, Braunholtz DA, Lilfotd R], Stevens AJ. Ethical issues in the design and conduct of cluster tandomised controlled trials, BM] 1999; $318: 1407.9$.

34. Brink W van den, Hendriks VM, Blanken P, Koeter MWJ, Zwieten BJ van, Re JM van. Medical prescription of heroin to treatment resistant heroin addicts: two randomised controlled trials. BM] 2003; 327: 310-5.

35. Brink W van den, Hendriks VM, Blanken P, Ree JM van. Het Nederlandse onderzoek naar de effectiviteic van heroine op medisch woorschrift; achtergronden, onderzoeksopzet en eerste ervaringen. Ned Tijdschr Geneeskd 2000; 144: 108-12. 



\section{Summary}

In Chapter 1 the topic of this thesis is introduced. The cause for this thesis was a discussion in the mid-Nineties in the Netherlands about the usefulness and ethical acceptability of pre-randomization in medical research. Triggered by the conviction that pre-randomization is useful in specific circumstances and against the background that it is often rejected on invalid grounds, the present study into the applicability and acceptability of pre-randomization in study designs was started.

Marvin Zelen first described the use of pre-randomization in study designs in 1977. The essence of this design is that potential participants are randomized before informed consent is obtained. This sequence is contrary to the conventional design in which informed consent is priot to randomization. Interventions in prerandomization designs can be applied in individuals or on the level of units, of clusters. Besides, after randomization, giving information about the intervention, and obtaining informed consent, can be done only in the experimental group (the so called single-consent design) or both in the expetimental group and in the reference group (the so called double-consent design).

Ethical principles for conducting scientific research are formulated in the Declatation of Helsinki and a sound informed consent procedure for participants entering a trial is one of the major tools to respect participants' autonomy. Nowadays, (bio)medical research projects involving human subjects must be reviewed by a research ethics committee and in that way study participants' rights are being protected.

Chapter 2 explores the history and principles of informed consent. Since World War II, a variety of protective measures have been introduced for persons receiving medical care or being involved in scientific research. "The concept of informed consent has been accepted as a standard. In experimental therapeutic research, informed consent still causes controversies; individual benefit being weighed against general benefit. In various types of observational research for many ethicists informed consent is becoming an absolute precondition to participate in a study. Observational epidemiological research on the basis of existing data is endangered. Both the Declaration of Helsinki and the Personal Registration Act state that, under certain circumstances, it may be possible to deviate from the requirement of obraining individual informed consent. A medical ethics committe could then give permission for such a research project. Informed consent should not be an end in itself, but a means to protect the individual. and to guarantee his/her right to autonomy. Insisting on informed consent in situations where autonomy is respected and no harm or burden is caused, seems to defy the purpose of informed consent. 
Chapter 3 gives a comprehenswe overwicw of the use of randomized consent designs (i.e. pre-randomization designs) since 1977. "Three types of randomized consent designs ate distinguished, ranked according to the extent to which prarticipants are informed about treatment options. Databases were searched for eligible studies and data were extracted on country of study conduct, year of commencement, area of medicine, type of design, reason(s) for use, details on approval by a research ethics committee, the index and reference interwention, nature of endpoints, and details on data collection. Furthermore, for each trial, the rates of not-compliance and loss to follow-up were registered by treatment arm. The thee types of randomized consent designs were compared as to differences between the rates of non-compliance and loss to follow-up in the separate trial arms. The number of trials in which a randomized consent design was used is very small $(n=50)$; over the years a shift from medical to behavioral type of interventions was seen. In $22 \%$ of the studies problems expected with the tandomization procedure was reported as the main reason for using a randomized consent design. In $65 \%$ of the studies the non-compliance in the index group is larger than in the reference group. In the majority of the studies the use of a randomized consent design was unjustified and the use of these designs should be testricted to studies ith which contamination is otherwise unavoidable.

Chapter 4 illustrates the use of a randomized consent design (Zelen design) by means of a fictitious heroin-provision experiment in badly integrated long-term addicts. Compared with the traditional clesign, the Zelen design probably reduces non-compliance and dropout considerably, thus increasing validity. Depending on the study population, the Zelen design may teduce study precision. However, in a trial aimed at badly integrated addicts the Zelen design can be conducted without loss of precision because baseline measurements will only weakly correlate with offect measurements. The Zelen design as an alternative fot a conventional mandomized design should be considered if: (a) the experimental intervention is attractive for all potential participants, and (b) the reference group will recerve standard treatment. If these conditions are met, the choice between the two designs depends on weighing the loss of valtidity due to selective dropout and noncompliance in the reference group against the potential loss of precision due to not pertoming a baseline measurement.

Additionally, the ethical problem of studying participants without their consent was solved by a stight modification of the Zelen design in which the sampling of a reference group is postponed.

The acceptation of this modified randomized consent design and Zelen's randomized consent design by members of research ethics committees in the United Kingdom, and in the Netherlands is studied in chapter 5 . In a postal survey 
the fictitious heroin-provision experiment was used as a vehicle to demonstrate both randomized consent designs.

In both the UK and the Netherlands, the modified tandomized consent design appears more acceptable than the nandomized consent design, with tespect to ethical and judicial aspects. The overall rejection rate of the randomized consent design was $66 \%$ in the UK and $59 \%$ in the Netherlands. However, the modified randomized consent design was rejected by $47 \%$ and $41 \%$ in the two countries, respectively. To increase consistency in the way research ethics committees handle study protocols, a discussion about the use of randomized consent designs appears necessary.

Chapter 6 presents guidelines for the optimal application of pre-randomization designs. For this purpose, designs in which pre-randomization is used are outlined, methodological considerations to choose berween conventional designs or prerandomization designs are described, and relevant ethical and judicial aspects are discussed. In specific situations, based on well-defined indications and requirements, pre-randomization designs have an essential contribution to achieve evidence-based medicine. In general, every design should be based on a bahance between maximizing the potential for patient autonomy and minimizing the bias caused by contamination. Although general rules are important, an independent research ethics committee should decide whether this balance is acceptable.

Chapter 7 reflects on the main findings and addresses some general issues concerning the acceptability and applicability of pre-randomization in study designs. In general, pre-randomization is justified if the study has a clinically relevant objective, it is likely that the study will lead to important new insights, a valid evaluation of the effects is impossible using a conventional randomized design, the informed consent procedure bears no potential harm to participants, at least usual care is offered to participants in the reference group, and the study protocol is approved by an independent research ethics committee. This study shows that, methodologically, pre-randomization is useful when an attractive or preferable experimental treatment is involved, the reference is the standard treatment, the use of a sham procedure or placebo cannot be used, and using prerandomization can minimize bias caused by contamination. Besides, in case of a single-consent design the choice depends on weighing the loss of validity due to selective dropout and non-compliance in the reference group against the potential loss of precision due to not performing a baseline measurement.

Banning pre-tandomization designs implies that knowledge about the effectiveness of many public health, medical, and clinical interventions will not be available. A number of potentially effective interventions will not be introduced because of the lack of evidence. Furthermore, the use of established, (possibly) 
ineffective intervention will be continued without evidence that the benefit prevails the harm.

In general, pre-randomization needs a reappraisal and for this purpose ethical, methodological, and judicial requirements must be discussed without prejudices, but based on valid arguments. This thesis may contribute to this discussion. 


\section{Samenvatting}

Hoofdstuk 1 beschrijft de aanleiding voor het schrijven van dit proefschrift en introduceert het onderwerp. De aanleiding is terug te voeren naar discussies, onder andere in de vakliteraturur, over de bruikbaarheid en de darmee samenhangende ethische toelaatbaarheid van pre-randomisarie. Bij de meest toegepaste manier van onderzoek, waarbij twee of meerdere groepen worden vergeleken, worden potentiële deelnemers geïnformeerd over het onderzoek, watua toestemming voor deelname wordt gevraagd (informed consent). Daatna worden de deehnemers op basis van loting (randomiseren) toegewezen aan een van de onderzoeksgroepen. Pre-randomisarie draait dit principe om: potentiële deelnemers worden eerst gerandomiseerd over de onderzoeksgroepen en daama wordt aan de experimentele groep, of aan alle groepen toestemming gevtaagd waarbij meestal geen informatie wordt gegeven over de andere groep.

Het meest bekende onderzoeksdesign waarbij pre-randomisatic wordt toegepast is in 1977 door Marvin Zelen geïntroduceerd. Dit design is bekend als het Zelen design. Hierbij wordt na randomisatic alleen aan personen in de experimentele groep toestemming gevraagd. De andere vormen van pre-randomisatie waatin na randomisatie aan alle deelnemers in verschillende variaties toestemming wordt gevraagd, zijn ontworpen om tegemoet te komen aan ethische bezwaren. Prerandomisatie wordt ook gebruikt indien interventies op groeps- of clusterniveau worden uitgevoerd en dientengevolge deze clusters worden gerandomiseerd in plaats van individuen. Voorbeelden van clusters zijn scholen, wijken, huisartspraktijken en gezondheidszorgvoorzieningen.

Essentieel bij de opzet van een onderzoek is de zorgvuldigheid waarmee proefpersonen worden geinformeerd en toestemming voor deelname wordt gevraagd. Dit is in alle gevallen noodzakelijk indien de autonomie van de persoon in het geding komt, of lichamelijke, emotionele of sociale schade kan worden berokkend. Om proefpersonen te beschermen is in ethische codes, zoals de Verklaring van Helsinki, vastgelegd waaraan wetenschappelijk onderzock moet voldoen. Experimenteel onderzoek waarbij mensen worden betrokken kan niet worden uitgevoerd zonder toestemming van een onafhankelijke commissie. In Nederland zijn dit de medisch ethische toetsingscommissies, die bij wet zijn ingesteld. In het buitenland bestaan vergelijkbate commissies, zij het op basis wan andere wer- of regelgeving.

In hoofdstuk 2 wordt nader ingegaan op de geschiedenis en de betekenis van informed consent. Sinds de Tweede wereldoorlog zijn allerlei beschermende maatregelen getroffen voor mensen die medische zorg ontvangen, of worden betrokken in een wetenschappelijk onderzoek. Steeds vaker werd geinformectde 
welowerwogen toestemming (informed consent) ingevoerd als standaard. In het experimenteel therapeutisch onderzoek zorgt informed consent nog steeds voor controversen, waarbij het nut voor het individu tegenover het algemene nut wordt geplaatst. Ook bij alle vormen van observationeel onderzoek lijkt informed consent cen dermate absolute eis te worden, dat epidemiologisch onderzoek op basis van bestaande gegevens onmogelijk dreigt te worden. Volgens zowel de Verklaring van Helsinki als de Wet persoonsregistratie is het onder bepaalde omstandigheden mogelijk af te wijken van het vragen van individuele informed consent. Toestemming voor onderzoek zou dan gegeven kunnen worden door een commissie van toezicht of een medisch-ethische commissie.

Informed consent dient geen doel op zich te zijn, maar een middel tot bescherming van proefpersonen en waarborging wan het recht op autonomie.

Hoofdstuk 3 gaat in op het vóórkomen van pre-randomisatie in gepubliceetd onderzoek sinds de introductie van deze wijze van opzet van onderzoek door Marvin Zelen in 1977. Door middel van systematisch zoeken in veel gebruikte literatuurbestanden werden slechts 50 studies gevonden waarbij pre-randomisatie werd toegepast. Over de jaren heen verschoof de toepassing van medische interventies (vooral bij oncologische trials) naar gedragsmatig interventies (zoals bijvoorbeeld advies over een andere levenswijze). In éénvijfde van het aantal studies waren verwachte problemen met randomiseren de reden om prerandomisatie toe te passen.

Bij de 50 gevonden onderzoeken werd nagegaan in hoeverre de proefpersonen afweken van de afgesproken richtlijnen (non-compliance) en hoeveel percent van de deelnemers hun deelname stopten, waardoor geen metingen konden worden gedaan (loss to follow-up). Zowel non-compliance als loss to follow-up kunnen het resultaat van een onderzoek negatief beïnloeden en de interpretatie en daarmee de toepasbaarheid van de resultaten bemoeilijken. Het blijkt dat in de meerderheid wan de studies de mate van non-compliance en loss to follow-up groter is in de experimentele groep dan in de controle groep. Dit is opvallend omdat prerandomisatie juist is bedoeld om non-compliance en loss to follow-up te vootkomen, vooral in de controle groep, ervan uitgaande dat vanwege de tantrekkelijkhetd van de interventie de proefpersonen in de experimentele groep graxg (blijven) meewerken. De conclusie lijkt gerechtvaardigd dat in de gevonden studies her toepassen van pre-randomisatie niet de juiste keuze is geweest.

Hoe pre-randomisatie wel verantwoord kan worden roegepast wordt beschreven in hoofdstuk 4. De toepasbaarheid van pre-randomisatie (Zelen design) wordt geillustreerd aan de hand van een fictief onderzoek naar de effectiviteit van het verstrekken van heroine aan langdurig verslaafde en verloederde druggebruikers. Bij een dergelijk onderzoek is het geven van een placebo niet mogelijk en is een 
andere vorm van blindering anguewezen. Het Zelen design is bruikbart voor een dergelijk onderzoek omdat het werstrekken wan hetoine kan worden gezien als een aatrekkelijke interventie en uitwal en non-complimnce in de controlegroep kan worden vermeden omdat deze deelnemers niet worden geinformeend over de interventie. Daarnaast is an de roorwaarde voldaan dat de controle groep de best beschikbare standaatdbehandeling krijgt en een beginmeting niet noodzakelijk is.

Om tegemoet te komen an vooral ethische bewwaten tegen het 2 eat design, wordt in dit hoofdstuk een alternatief design gepresenteen dat methodologisch gelijk is aan het Zelen design, maar waabij de steekproeftrekking woor de controlegroep wordt uitgesteld. Onderzoekers en ethici die we beide desingns tet beoordeling voorhielden vonden het alternatieve design meer acceptabel dan het: Zelen design.

Dit gegeven was aanleiding het Zelen design en het alternatieve design ter beoordeling voor te leggen aan leden van medisch ethische toetsingscommissies. Dit onderzoek wordt beschreven in hoofdstuk 5. Een enquête werd gestuurd nat: alle medisch ethische commissies in Nedetland (77) en in Groot-Brittannië (240). Leden die deskundig waren op het gebied van de ethiek, (gezondheids)reclat, methodologie of klinische praktijk werd gevraagd of $z \mathrm{e}$ het $\mathrm{Zelen}$ design en/of het alternatieve design ethisch, juridisch en/of methodologisch acceptabel vonden. De beoordeling vond plaats aan de hand van een fictief heroine-verstrekkingsexperiment, zoals beschreven in hoofdstuk 4. Zowel in Nederland als in GrootBrittannië bleek het alternatieve design wat betreft ethische en juridische aspecten meer acceptabel dan het Zelen design. Naar onze mening is et geen methodologisch verschil tussen beide designs en zouden beide designs gelijk beoordeeld moeten worden. Blijkbaat spelen vooral ethische en juridische argumenten een rol bij de beslissing of pre-randomisatie toelatbar is. Om meer consistentie te krijgen in de beoordeling van onderzoeksprotocollen door medisch ethische toetsingscommissies is een discussie angewezen warbij an de hand van de voor- en nadelen ran pre-randomisatie consensus wordt bereikt, bijwoorbeeld door middel van een Delphi consensus techniek. Het lijkt onwenselijk dat onderzoekers in essentie gelijke onderzoeksdesigns op een andere wijze formuleren met als doel meer kans te maken op goedkeuring doot een medisch ethische commissie.

In hoofdstuk 6 worden richtijnen gepresenteetd voor de juiste toepassing van prerandomisarie. Hierbij wordt onderscheid gemaakt tussen pre-randomisatic wararbij individuele proefpersonen een interventie krijgen en pre-tandomisatie waarbij de interventie op clusterniveau plaatsvindt. In het eerste geval worden individuen gerandomiseerd en in het andere geval clusters zoals schoolklassen, ziekenhuizen of huisartsptaktijken. Belangrijk. bij de keuze tussen een conventioneel design en 
pre-tandomisatic designs ziln methodologische overwegingen, waarbil wordt nagegan wat de invloed is van non-compliance en loss to follow-up op de wetenschappelije kwaluteit (nteme validiteit) van het onderzoek. De mate waatin deze arantastingen wan het protocol (door contaminatie) kunnen optreden kan blindering noodzakelik maken. Blindering zorgt er voor dat proefpersonen, onderzokers en degene die de effecten meten niet weten welke behandeling is toegewezen. Als het tisico op contaminatie laag wordt ingeschat, of als het gebruik van een placebo kan zorgdragen voor blinderen, dan is een conventioneel onderzoeksdesign te overwegen. In andere gevallen kan het gebruik van prerandomisatie voor de juiste blindering zorgen en kan aan de hand van een stroomschema worden bepaald welk soort pre-tandomisatie design bruikbaar is.

Daarnaast zjjn ethische en juridische overwegingen van belang bij de opzet van onderzoek. Enerzijds behoort een proefpersoon/patiënt de best mogelijke behandeling te krijgen, anderzijds draagt een proefpersoon/patiënt bij aan de vooruitgang van de (medische) zorg. Dit respectievelijk individueel- en collectief ethisch belang dient in evenwicht te zijn. De manier waarop de informed consent procedure wondt uitgevoerd en het moment waarop informed consent, al dan niet, wordt gevragd is tevens onderhevig an de juiste afweging. In elk onderzoeksdesign moet worden gezocht naar de juiste balans tussen het maximale behoud wan de autonomie van de proefpersoon en het minimaliseren van de vertekening van de resultaten veroorzaakt door contaminatie. Of in een onderzoeksprotocol deze balans wordt gevonden, is ter beoordeling aan medisch ethische toetsingscommissies.

Hoofdstuk 7 gat in op de belangrijkste bevindingen. Pre-randomisatie is toepasbaar als de onderzoekswraag relevant is, de resultaten kunnen leiden tot belangrijke nieuwe inzichten, het onderzoek niet kan worden uitgevoerd met een conventioneel design, de deelnemers in de controlegroep tenminste de standaard zorg krijgen en het onderzocksprotocol is goedgekeurd door een medisch ethische toetsingscommissie. "Tevens tonen de bevindingen aan dat methodologisch gezien pre-tandomisatic is angewezen indien de experimentele interventie aantrekkelijk is voot de deelnemers, blindering op een andere maniet niet mogelijk is en het gebnuk van pre-randomisatie de vertekening van de resultaten, veroorzaakt door contaminatie, kan minimaliseren. Zodoende kunnen pre-randomisatie designs een essentiele bijdrage leveren aan de ontwikkeling van het evidence-based (be)handelen. Het witbannen van pre-randomisatie zow betekenen dat gegevens over de effectiviteit van veel medische- klinische- en openbaar gezondheidszorginterventies niet beschikbaar komen. Potentieel effectieve interventies blijwen onbekend en mogelijk ineffectieve interventies kumnen blijven bestan vanwege het ontbreken van bewijs van werkzaamheid. Daarom is het van belang dat medisch ethische toetsingscommissies door de beoordeling van 
onderzoeksprotocollen de gezondheidszorg en de volksgezondheid blijven beschermen. Dit onderzoek toont aan dat deze commissies discussies over minder conventionele designs, zoals pre-randomisatie designs, niet uit de weg gaan.

In algemene zin verdient de toepassing van pre-randomisatie in onderzoeksdesigns een herwaardering om zodoende bil te dragen an een betere kwaliteit van wetenschappelijk onderzoek. De discussie hierover dient vrij te zijn van ethische; juridische en methodologische vooroordelen. De inhoud van dit proefschrift kan aan deze discussie bijdragen. 



\section{Dankwoord}

Dankzij de hoogwaardige academische begeleting was her schrijven van dit proefschrift een uitdagende, boeiende, leerzame en zelfs plezierige bezigheid. Mijn promotores prof. dr. Ferd Sturmans en prof. dr. André Knottnerus en mijn copromotor dr. Gerben ter Riet, hebben een buitengewoon belangrijke bijdrage geleverd aan het tot stand komen van dit proefschrift, waarvoor veel dank. De bijdrage van ir. Fons Kessels was onmisbaar. Zonder aijn inspanningen was dit proefschrift er niet.

Beste Fons, onze samenwerking begon midden jaren negentig van de vorige eeuw, vooral vanwege jouw betrokkenheid bij de opzet van een heroineverstrekkingsexperiment. Bij de koffiehoek van de capaciteitsgroep epidemiologie werden de eerste vernieuwende inzichten bedacht. Jouw $2 \mathrm{e}$ auteursschap bij alle hoofdstukken zegt veel over je bijdragen die je sindsdien hebt geleverd en je betrokkenheid, waarvoor ik bijzonder veel waardering heb. Jouw verhelderende kijk op de wijze hoe onderzoek moet worden opgezet is bijzonder. Ik heb van jou geleerd op te schrijven wat echt belangrijk is en leuterverhalen te vermijden. Of de balans tussen leesbarheid en compactheid is gelukt, laten we aan de lezer over. $\mathrm{OP}$ vele zaterdagen hebben we aan hoofdstukken gesleuteld. Soms was de productie een half A4-tje, maar dan stond er ook wat. In mijn dank voor je buitengewone bijdrage en steun wil ik ook je vrouw Tiny betrekken, die mij in Wijlte zo vaak over de vloer heeft zien komen.

Beste Ferd, jii hebt mij zo'n tien jaar geleden gevraagd voor de protocolontwikkeling voot een heroine-verstrekkingsexperiment, mijn oorspronkelijk promotieonderwerp. Het is zeer spijtig dat we niet de gelegenheid hebben gehad aan te tonen dat het Zelen design hierbij werkt, omdat de uirvoering aan Amsterdam werd toegewezen en niet aan Rotterdam. Jouw bijzondere gedrevenheid heeft mij gemotiveerd een ander pad te kiezen en prè-randomisatie in breder perspectief te plaatsen. Als we elkaar in Maastricht spraken kon ik weer weken vooruit.

Beste Gerben, je hebt altijd veel meegedacht en meegeschreven en als copromotor je tol uiterst zorgvuldig vormgegeven. Ik heb veel baat gehad bij je nauwgezet commentaar, Engelse correcties en aanscherping van inzichten. Dit heeft naar mijn mening de kwaliteit van mijn proefschrift behoorlijk bevorderd.

Beste André, je bent in een latere maar cruciale fase als promotor bij mijn onderzoek betrokken geraakt. Dat heeft duidelijk zijn vruchten afgeworpen. Het 
geheel is nar een abstractet niveau getil, het venin, dat steeds maar weer in de teksten terug blet komen, is enuit en je genuanceerd denken over complexe zaken. makte die voor mij inzichtelijk.

Het schrifven van een wetenschappelike verhandeling naast een niet-academische baan, kan gedoemd zijn te mislakken. Dat is voorkomen door de betrokkenheid van Jacques Lucieer, hoofdinspecteur bij de Inspectie voor de Gezondheidszorg; die mij de gelegentheid heeft geboden begin 2003 studieverlof te nemen om een nicuwe start te maken met de afronding van mijn proefschrift. Voor deze geboden mogelijkheid wil ak ook Herre Kingma, inspecteur-generaal, Ineke Bik-Bakker, toenmalig clustercoördinator en Jan Tjoeng, rijksinspecteur, bedanken. Tevens dank aan alle andere collega"s van de inspectie voor hun niet aflatende belangstelling.

Ik ben bijzonder blij dat Sjef Wijnen en Gerard Swaen mij als paranimfen willen bijstaan. Beste Sjef, vtiend in alle tijden, jouw jarenlange motiverende en ktitische houding heeft niet alleen mijn wetenschappelijk werk goed gedaan. Beste Gerard, tijdens de vele lange wandelingen in de Ardennen heeft jouw nuchtere kijk op ogenschijnlijk voor mij lastige overwegingen, mil weer op het goede spoor gezet.

Bij het onderzoek zijn veel leden van medisch ethische commissies betrokken. Ik ken ze niet persoonlijk, maar wil ze toch bedanken voor hun deelname en hun op deze wijze votmgegeven bijdrage an een mogeli.jk tockomstige discussie over de bruikbaatheid wan pre-randomisatie. Jos Kleijnen van de universiteit van York, heeft het mogelijk gemaakt dat ilk het onderzoek in Groot-Brittannië kon uitvoeren. Ook wil ik hierbij Annemic Mordant bedanken voor haar ondersteuning

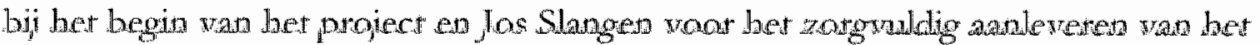
adressenbestand. Pieter Leffers is altijd de verbindende schakel gebleven met de capaciteitsgroep tipidemiologie.

Soms moet je de juiste toon tieffen, bij Combo Picon altijd! Desondanks zotgt dit zootje ongeregeld er wekelijks voor dat jazz ontstressed. Ja ja, het boekje is klaar, op nar het volgende slachtoffer!

Thum Aarts heeft op onnavolgbare wijze mijm proefschrift opgemaakt. Beste Thum, ik kon je tempo niet bijbenen, maar het is toch allemaal goed gekomen. Merci.

Beste Audrey, het verbaast mij nog steeds dat je binnen de kortste keren de essentie van de abstracte inhoud van mijn proefschrift beeldend wist om te zetten in het ontwerp van de omslag. 
Tenslotte, hoe je het ook draait of keert: zonder stabiel thuisfront geen proefschrift. Lieve Vera, je hebt mij nooit een strobreed in de weg gelegd. Dat beviel zo goed dat ik zou zeggen, ga er voonal mee door! Lieve Julia, maak je docenten maar gek met de term 'evidence-based', daat pluk je in je toekomstig werk alleen maar de vruchten van. 



\section{Curriculum Vitae}

Ron Schellings was born on April 7, 1958 in Maastricht, the Netherlands. After completing secondary school he studied psychiatric nursing, followed by social psychiatric nursing at the Academy of Health Care in Sittard. He obtained his degree in 1982 and worked at the Academic Anxiety Center (psychiatric hospital Vijverdal, Maastricht) for seven years. From 1989 until 1992 he worked at the Consultation bureau of Alcohol and Drugs Limburg. After studying economics at Maastricht University in 1988-89, he started studying Health Science at the same University. He graduated in 1992 and worked as a researcher at the department of Epidemiology of Maastricht University, were he was involved in a study on substitute care for long-term psychiatric patients, and in the protocol development for a heroin-provision experiment. This project laid the foundation for the subject of this thesis. Besides working at the department of Epidemiology, he worked at the Institute of Psycho-Social and Psycho-Ecological Research (training program for older adults with mental retardation) and the department of Medical Sociology (Alcohol and Drugs Monitoring). Since 1999 he works as an inspector of health care at the Health Care Inspectorate of the Public Health Supervisory Service of the Netherlands. He is in charged of mental health care and since 2004, in particular, of the forensic psychiatry. 